\title{
MONITOREO DE PROCESOS MEDIANTE \\ TÉCNICAS DE ENTROPÍA APROXIMADA
}

Tesis que para obtener el grado de:

Doctora en Ciencias (Ingeniería Química)

Presenta:

M. en C. Ernestina Moreno Rodríguez 


\section{A91

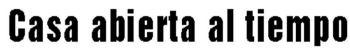

Universidad Autónoma Metropolitana Unidad Iztapalapa

División de Ciencias Básicas e Ingeniería

Departamento de Ingeniería de Procesos e Hidráulica

Posgrado en Ingeniería Química

\section{MONITOREO DE PROCESOS MEDIANTE \\ TÉCNICAS DE ENTROPÍA APROXIMADA}

Tesis que para obtener el grado de:

Doctora en Ciencias (Ingeniería Química)

Presenta:

M. en C. Ernestina Moreno Rodríguez

Asesor:

Dr. José de Jesús Álvarez Ramírez

México, D.F., a Septiembre de 2013 



\section{A41 \\ ․․ \\ UNIVERSIDAD AUTÓNOMA METROPOLTANA}

Pecha : $26 / 09 / 2013$
Psgina $=$
$1 / 1$

La universidad Autonoms Metropolitana extiende la presente CoxstavcIA DE PRESENTACION DE DISERTACIÓN FÚBICA de DOCTORA BN CIENCIAS (IMOENIERIA QUINICA) te la alumna BRNESTIKh MORBNo RODGIGUEZ, matricula 209382045, quien cumplio con los 348 erfditos correspondientes a las unidades de engef̂nza aprendizaje del plan de eotudio. Con fecha veintisiete de eeptienbre del 2013 presents 1 a DEPENSA de su DISBRThCI6n POBLICA tuya denominscibn es:

MONIYOREO DR PROCESOS MEDIANTE TECXICAS DE ENTROEIA hFROXIMADA.

Cabe mencionar gue la aprobacicn tiene un valor de 100 erfditos y el programa consta de 528 er6attog.

El jurado del examen ha tenido a bien otorgarle la calificacion de:

APROBAR

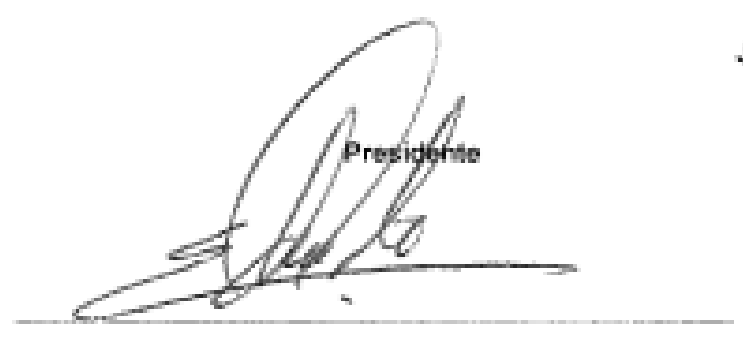

DR. BDUARDO SALVADOR PEPEZ CIBMEROS

JURADO

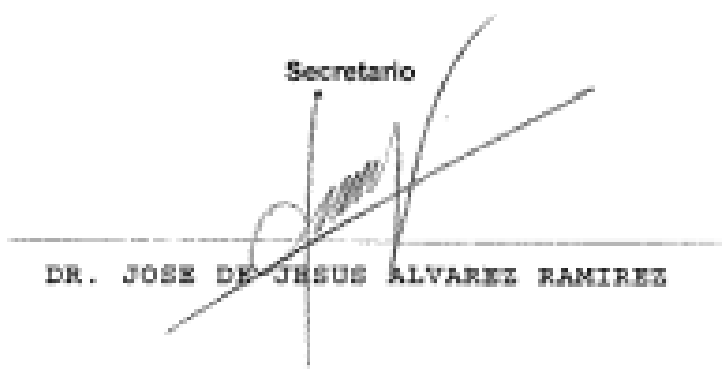

Voes 1

Vooal
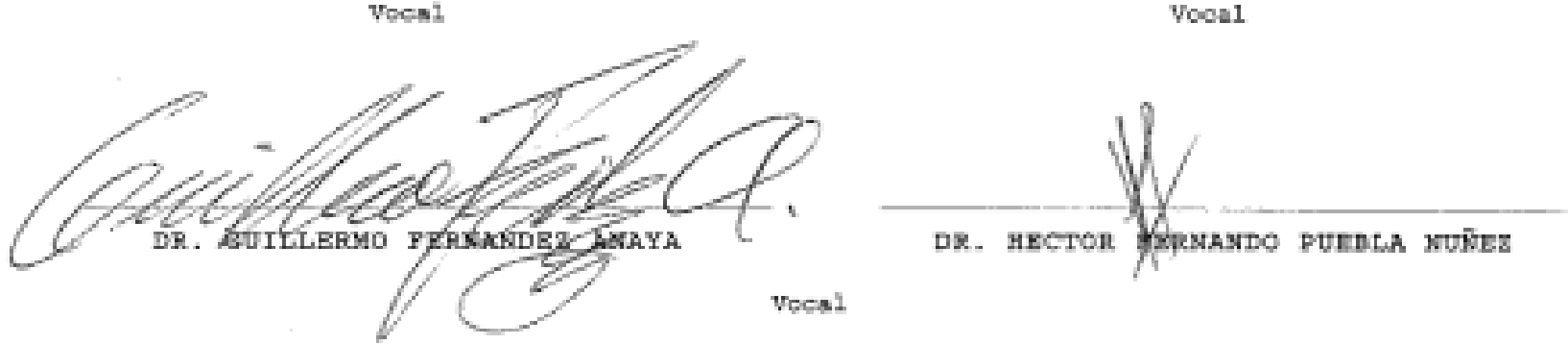

Veces

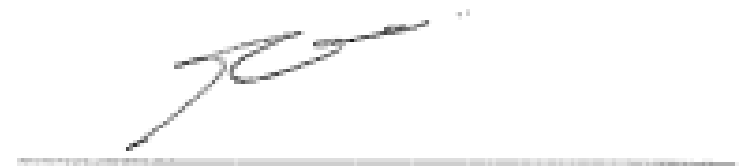

DR, BASILIO DEL MURD CURLIAR

UNIDAD IZTAPALAPA

Coordinación de Sistemas Escolares

Av. Sim Fafael Atlixco 188, Col. Vicentina, Mexico, DF, CP 09340 Apte. Pestal 555-320-9000, Tels, 5804-4880 y 5804-4883 Fax: 5804-4876 


\section{Agradecimientos}

A mi asesor, el Dr. José de Jesús Álvarez Ramírez, por su dirección en el trabajo de investigación y por brindarme su apoyo durante mis estudios de doctorado.

Al jurado quienes como revisores dedicaron tiempo para la revisión y contribuyeron con sus comentarios a mejorar la calidad de la presente tesis.

- Dr. Eduardo Salvador Pérez Cisneros. (UAM-lzt)

- Dr. Héctor F. Puebla Núñez. (UAM-Azc)

- Dr. Guillermo Fernández Anaya. (UIA)

- Dr. Basilio del Muro Cuéllar. (IPN-ESIME)

A mi familia, mi esposo Jesús Alejandro quien siempre me brindo su apoyo y me alentaba a seguir superándome durante mis estudios. A mi hijo y mi bebé que viene en camino, quienes son unas bendiciones de dios y que siempre me dan energía para seguir adelante.

A mis papás y mis hermanos quienes siempre confiaron en mí, y me alentaban para seguir esforzandome.

Finalmente, al Consejo Nacional de Ciencia y Tecnología por su apoyo económico brindado. 


\section{Resumen}

Durante el control o la supervisión de procesos químicos pueden presentarse situaciones anormales o deficiencias de operación como consecuencia de inestabilidades dinámicas, perturbaciones, formación de productos de corrosión, ruido y/o fallas en los sistemas de medición, entre otras situaciones. Por consiguiente es necesario un sistema de monitoreo que sea capaz de manipular y extraer información adecuada de datos de procesos que pueden presentar comportamientos no lineales, no estacionarios y con ruido.

En el presente trabajo se utilizaron técnicas de entropía aproximada ( $A p E n)$ para definir criterios de fallas o inestabilidades durante el monitoreo de procesos químicos como son columnas de destilación y procesos de corrosión electroquímica. Por medio de algunas técnicas de entropía se evaluaron las fluctuaciones y/o regularidades en las series temporales de los sistemas en estudio considerando los efectos de tendencia, correlación temporal, y así como el ruido implícito en señales de procesos. Una de las técnicas de entropía empleada fue el análisis de entropía aproximada sin tendencia $(D E A)$, la cual considera que los sistemas dinámicos pueden ser afectados por dinámicas no estacionarias que afectan su tendencia en las series de tiempo. Además se utilizo el análisis de entropía aproximada multiescala (MEA)

para revelar información en múltiples escalas espaciales y temporales, donde se considera que los sistemas dinámicos estudiados pueden llegar a mostrar dinámicas complejas, los cuales se encuentran en completa aleatoriedad, lejos de la perfecta regularidad.

Los resultados obtenidos demuestran que durante el monitoreo de procesos, como son columnas de destilación o procesos de corrosión electroquímica, si una serie de tiempo presenta valores altos de entropía corresponderá a fluctuaciones importantes o irregularidades en el sistema dinámico. La información obtenida de los análisis de entropía permitió establecer un criterio de falla o inestabilidad a consecuencia de perturbaciones, cambios de operación, formación de picaduras o pérdida de metal, de acuerdo al sistema bajo estudio. 


\section{ÍNDICE GENERAL}

\begin{tabular}{|l|l|}
\hline Índice general I & I \\
\hline
\end{tabular}

\begin{tabular}{|l|l|l|}
\hline Índice de figuras & IV
\end{tabular}

\begin{tabular}{lll}
\hline Índice de tablas & VII
\end{tabular}

1. Introducción 1

1.1. Antecedentes . . . . . . . . . . . . . . . . . . 1

1.1.1. Monitoreo de procesos químicos . . . . . . . . . . . . . . . . . 4

1.1.2. Teoría de información . . . . . . . . . . . . . . . . . . . 6

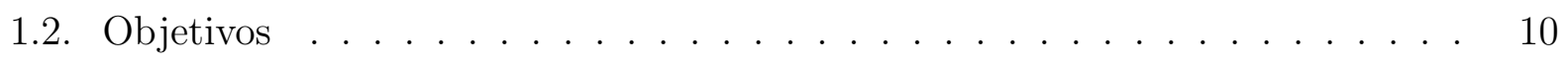

1.2.1. Objetivo general . . . . . . . . . . . . . . . 10

1.2.2. Objetivos particulares . . . . . . . . . . . . . . 10

1.3. Organización de la tesis . . . . . . . . . . . . . . . . . . 11

I Análisis de señales sintéticas y de difracción de rayos $X] 13$

\begin{tabular}{ll}
\hline 2. Señales sintéticas $1 / f^{\beta}$ & 14
\end{tabular}

2.1. Introducción . . . . . . . . . . . . . . . . . . . . 15 
Índice general

2.2. Metodología . . . . . . . . . . . . . . . . . . . . . 16

2.3. Resultados . . . . . . . . . . . . . . . . . . . . . . . . . 16

2.4. Conclusiones . . . . . . . . . . . . . . . . . . . . . 21

\begin{tabular}{ll}
\hline 3. Señales de intensidad de difracción de rayos $X$ & 23
\end{tabular}

3.1. Introducción . . . . . . . . . . . . . . . . . . . . . . . . . . . 23

3.2. Metodología . . . . . . . . . . . . . . . . . . . . . 26

3.3. Resultados . . . . . . . . . . . . . . . . . . . . . . 27

3.4. Conclusiones . . . . . . . . . . . . . . . . . . . . . . 32

\section{Monitoreo de columnas de destilación $y$ sistemas de} $\begin{array}{ll}\text { corrosión electroquímica } & 33\end{array}$

4. Columnas de destilación 34

4.1. Introducción . . . . . . . . . . . . . . . . . . . . . . . . 34

4.1.1. Factores que afectan la columna de destilación . . . . . . . . . . . 35

4.1.2. Variables a monitorear en columnas de destilación . . . . . . . . . . . 39

4.2. Metodología . . . . . . . . . . . . . . . . . . . . . . . . . . . . . 39

4.3. Resultados . . . . . . . . . . . . . . . . . . . . . . . . . . . 41

4.3.1. Análisis de entropía aproximada . . . . . . . . . . . . . . . . 41

4.3.2. Complejidad de señales . . . . . . . . . . . . . . . . . . . . 43

4.3.3. Perturbaciones en las condiciones de alimentación . . . . . . . . . . 46

4.4. Conclusiones . . . . . . . . . . . . . . . . . . . . . . . . . . . . 52

5. Corrosión electroquímica 54

5.1. Introducción . . . . . . . . . . . . . . . . . . . . . . 54

5.1.1. Monitoreo de la corrosión . . . . . . . . . . . . . . . . . 54

$5.1 .2 . \quad$ Corrosión uniforme . . . . . . . . . . . . . . . . . 56

5.1.3. Corrosión localizada . . . . . . . . . . . . . . . . . 57

$5.2 . \quad$ Metodología . . . . . . . . . . . . . . . . . . . . 58

\begin{tabular}{llc}
\hline Ernestina Moreno Rodríguez & Tesis Doctoral & Página II
\end{tabular} 
Índice general

5.3. Resultados . . . . . . . . . . . . . . . . . . . . . 60

5.3.1. Análisis visual del $E N$. . . . . . . . . . . . . . . . . . . . . . . . . 60

5.3.2. Análisis de entropía aproximada . . . . . . . . . . . . . . . . . . 62

5.4. Conclusiones . . . . . . . . . . . . . . . . . . . . . . . . . . 69

\begin{tabular}{ll}
\hline 6. Conclusiones & 71
\end{tabular}

\begin{tabular}{ll}
\hline Bibliografía & 74
\end{tabular}

\begin{tabular}{|ll}
\hline Apéndices & A-1
\end{tabular}

\begin{tabular}{|ll}
\hline A. Columnas de destilación & A-1
\end{tabular}

A.1. Casos de estudio . . . . . . . . . . . . . . . . . . . A A-1

A.2. $\operatorname{ApEn}(\tau)$ en función del tamaño de ventana $N_{w}$. . . . . . . . . . . . . . A-6

A.3. Entropía aproximada multiescala . . . . . . . . . . . . . . . . A-6

\begin{tabular}{ll}
\hline B. Entropía aproximada & B-1
\end{tabular}

\begin{tabular}{ll}
\hline C. Entropía aproximada sin tendencia & C-1
\end{tabular}

\begin{tabular}{|l|l}
\hline D. Entropía aproximada multiescala & D-1
\end{tabular} 
1.1. Señal de proceso $\ldots \ldots \ldots \ldots \ldots$

2.1. Ejemplos de algunas señales sintéticas de ruido $1 / f^{\beta} . \ldots \ldots \ldots$

2.2. $D E A$ para señales $1 / f^{\beta} \ldots \ldots \ldots \ldots \ldots \ldots \ldots \ldots$

2.3. Entropía aproximada multiescala de señales sintéticas $1 / f^{\beta}$. . . . . . . 19

2.4. DEA para el movimiento Browniano y su señal aleatoria. . . . . . . . . . . . 20

2.5. DEA para la señal de ruido blanco con acciones de control por (a) retroalimentación con amortiguación y (b) con retardos. . . . . . . . . . . . . 21

3.1. Intensidad de patrones $I(2 \theta)$ de rayos $X$ para alúmina calcinada a 200 y $500{ }^{\circ} \mathrm{C} .25$

3.2. (a) Intensidad de patrones $I(2 \theta)$ de rayos $X$ y (b) Patrones de entropía $E(2 \theta)$ de la señal de intensidad $I(2 \theta)$ para alúmina calcinada a $200^{\circ} \mathrm{C}$. . . . . . . 29

3.3. (a) Intensidad de patrones $I(2 \theta)$ de rayos $X$ y (b) Patrones de entropía $E(2 \theta)$ de la señal de intensidad $I(2 \theta)$ para alúmina calcinada a $500^{\circ} \mathrm{C}$. . . . . . . 30

3.4. Patrones de entropía $E(2 \theta)$ en función de la temperatura de calcinación para cuatro diferentes regiones angulares. . . . . . . . . . . . . . . . . . . 31

4.1. Localización de la línea $q$ para condiciones típicas de alimentación. . . . . . . 36

4.2. Relación de reflujo óptima . . . . . . . . . . . . . . . . . . . . . . 37

4.3. Características de operación en platos perforados. . . . . . . . . . . . . 38 
4.4. Diagrama esquemático de la columna de destilación binaria por etapas. . . . 40

4.5. Entropía aproximada $A p E n(\tau)$ como una función de la longitud del tamaño de ventana $N_{w}$, para cuatro diferentes escalas de tiempo $(\tau)$ para la señal de temperatura de la columna C. . . . . . . . . . . . . . . . . . . . . . . . . 42

4.6. Señal de temperatura en columna de destilación . . . . . . . . . . . . . . . . 44

4.7. Entropía a diferentes escalas $(\tau)$ de señales de temperatura para las secciones de rectificación y agotamiento de la columna C. . . . . . . . . . . . . . . . . 44

4.8. Entropía aproximada multiescala de la columna de destilación C. . . . . . . . 45

4.9. ApEn cuando se realizan perturbaciones en la composición de alimentación de la Columna C. . . . . . . . . . . . . . . . . . . . . . . . 48

4.10. Entropía aproximada multiescala de la columna de destilación C cuando se realizan cambios en la composición de alimentación. . . . . . . . . . . . . . . 49

4.11. ApEn en diferentes etapas de la columna cuando se llevan a cabo cambios en la composición de alimentación de la Columna C. . . . . . . . . . . . . . . . 51

4.12. ApEn cuando se realizan cambios en el flujo de alimentación en la Columna C. 52

4.13. ApEn cuando se llevan a cabo cambios de operación en la relación de reflujo y flujo de vapor de la Columna C. . . . . . . . . . . . . . . . . . . . 53

5.1. Serie de tiempo de (a) Voltaje y (b) Corriente del acero API X52 inmersa en solución acuosa $0.5 \mathrm{M} \mathrm{NaHCO}$ sin iones $\mathrm{Cl}^{-}$. . . . . . . . . . . . . . . . . . 60

5.2. Serie de tiempo de (a) Voltaje y (b) Corriente del acero API X52 inmersa en solución acuosa $0.5 \mathrm{M} \mathrm{NaHCO} 3$ con iones $\mathrm{Cl}^{-}$a una concentración $0.1 \mathrm{M}$. . . $\quad 61$

5.3. (a) Serie de tiempo de Voltaje y (b) Patrones de entropía $A p E n(\tau)$ de la serie de $E N$ de voltaje del acero $A P I X 52$ inmersa en solución acuosa $0.5 \mathrm{M}$ $\mathrm{NaHCO}_{3}$. . . . . . . . . . . . . . . . . . . . 63

5.4. (a) Serie de tiempo de Corriente y (b) Patrones de entropía $A p E n(\tau)$ para la señal de $E N$ de corriente del acero $A P I X 52$ inmersa en solución acuosa $0.5 \mathrm{M}$ $\mathrm{NaHCO}_{3}$. . . . . . . . . . . . . . . . . . . . . . . . . 64 
5.5. Valores de entropía a diferentes escalas de tiempo para la señal de $E N$ de (a) Voltaje y (b) Corriente del acero API X52 inmersa en solución acuosa 0.5M $\mathrm{NaHCO}_{3}$. . . . . . . . . . . . . . . . . . . 65

5.6. (a) Serie de tiempo de Voltaje y (b) Patrones de entropía $A p E n(\tau)$ para serie de $E N$ de voltaje del acero $A P I X 52$ inmersa en solución acuosa $0.5 \mathrm{M}$ $\mathrm{NaHCO}_{3}$ con iones $\mathrm{Cl}^{-}$a una concentración 0.1M. . . . . . . . . . . . . . . 66

5.7. (a) Serie de tiempo de Corriente y (b) Patrones de entropía para la señal de $E N$ de corriente del acero $A P I X 52$ inmersa en solución acuosa $0.5 \mathrm{M} \mathrm{NaHCO} 3$ con iones $\mathrm{Cl}^{-}$a una concentración $0.1 \mathrm{M} . \quad$. . . . . . . . . . . . . . . . . . . . 67

5.8. Valores de entropía a diferentes escalas de tiempo para la señal de $E N$ de (a) Voltaje y (b) Corriente del acero API X52 inmerso en solución acuosa 0.5M $\mathrm{NaHCO}_{3}$ con iones $\mathrm{Cl}^{-}$a una concentración $0.1 \mathrm{M} . . . . . . . . .68$

A.1. Entropía aproximada $A p E n(\tau)$ como una función de la longitud de la muestra $N_{s}$, para cuatro diferentes escalas de tiempo $\tau$ para la señal de temperatura de la columna C. . . . . . . . . . . . . . . . . . . . . . A-6

A.2. Entropía aproximada multiescala de la columna de destilación A. . . . . . A-6

A.3. Entropía aproximada multiescala de la columna de destilación B. . . . . . . . A-7 A.4. Entropía aproximada multiescala de la columna de destilación D. . . . . . . A-7 


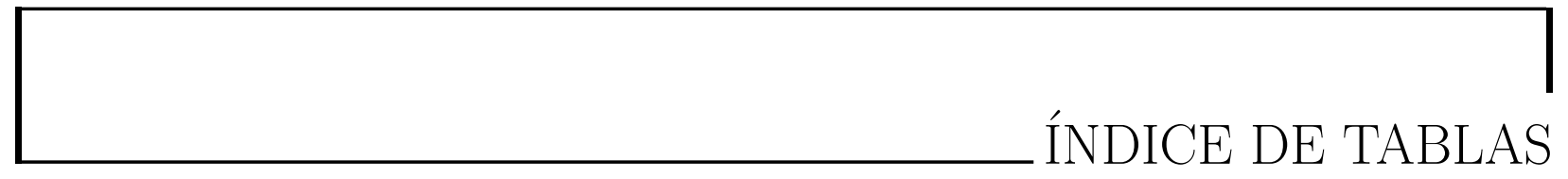

A.1. Datos en estado estacionario de la Columna A . . . . . . . . . . . . . . . A-2

A.2. Datos en estado estacionario de la Columna B . . . . . . . . . . . . . A-3

A.3. Datos en estado estacionario de la Columna C . . . . . . . . . . . . . . . A-4

A.4. Datos en estado estacionario de la Columna D . . . . . . . . . . . . A A-5 


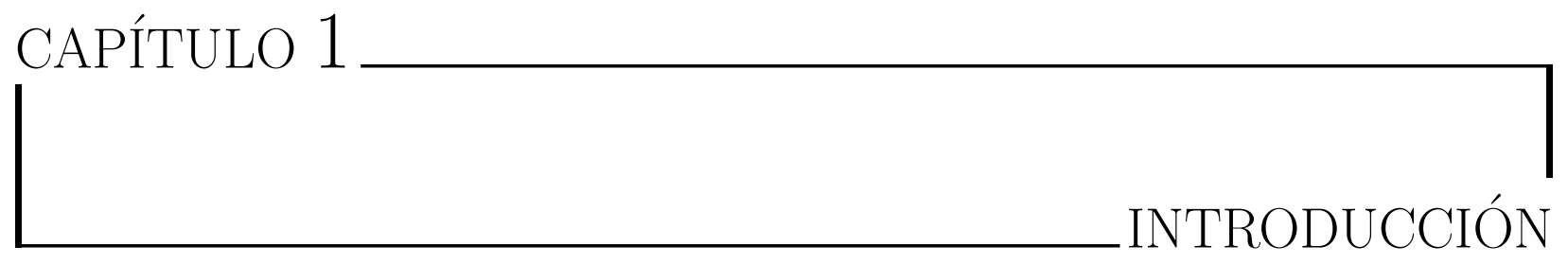

En este Capítulo 1 se presenta una introducción general de la investigación doctoral. En la Sección 1.1 se muestran los antecedentes bibliográficos de los temas mas relevantes a la investigación como son el monitoreo de procesos químicos y teoría de información. Posteriormente en la Sección 1.2 se describen los objetivos tanto el general como los específicos alcanzados en el presente trabajo de investigación. Finalmente en la Sección 1.3 se describe como se encuentra organizada la presente tesis doctoral.

\subsection{Antecedentes}

En plantas industriales se pueden llegar a manejar numerosas variables de proceso, las cuales deben de ser mantenidas dentro de límites específicos para una correcta operación del mismo. Sin embargo, desviaciones entre las variables claves más allá de los límites adecuados de operación podrían llegar a tener consecuencias directamente relacionadas con criterios económicos, calidad de producción, nivel de seguridad de la planta, medio ambiente, calidad del producto o rentabilidad. Por lo cual, la detección oportuna de eventos que pudieran afectar el rendimiento en los sistemas de operación requieren del monitoreo continuo de los mismo. 
Introducción

El monitoreo de procesos juega un papel clave en asegurar que el rendimiento del proceso cumpla con los objetivos de operación. Los objetivos generales del proceso de monitoreo son (Seaborg y col., 2004):

- Monitoreo de rutina. Asegurar que las variables del proceso se encuentren dentro de límites específicos.

- Detección y diagnostico. Detectar procesos de operación anormales y diagnosticar la causa.

- Monitoreo preventivo. Detectar tempranamente situaciones anormales, de tal forma que la acción correctiva se lleve a cabo antes de que el proceso se vea seriamente afectado.

Los procesos de operación anormales pueden ocurrir debido a una gran variedad de razones como son: problemas en equipos (fallas en intercambiadores de calor), instrumentos en mal funcionamiento (fallas en el control de válvulas, sensores inexactos), perturbaciones inusuales (reducción de la actividad catalítica, pequeños cambios en la composición de alimentación), entre otras causas (Seaborg y col., 2004).

Hoy en día dar seguimiento a un proceso determinado involucra el manejo de una gran cantidad de variables lo que se traduce de igual forma en una gran cantidad de información. La cual puede ser recopilada a través de series temporales que describen el comportamiento de variables discretas durante un lapso de tiempo determinado. En la Figura 1.1 se muestra que series temporales pueden esconder estructuras o correlaciones entre variables, que derivan en una valiosa fuente de información que podría ayudar a mejorar la operación y eficiencia del proceso.

Sin embargo, el análisis de series temporales puede involucrar el manejo de señales cuyo comportamiento presenta tendencia (tipo lineal o no lineal), variación cíclica, estacional o irregular. Además, debido a condiciones de operación el ruido está implícito en los procesos, por lo tanto se deben considerar sus efectos durante el análisis de señales. 
Introducción

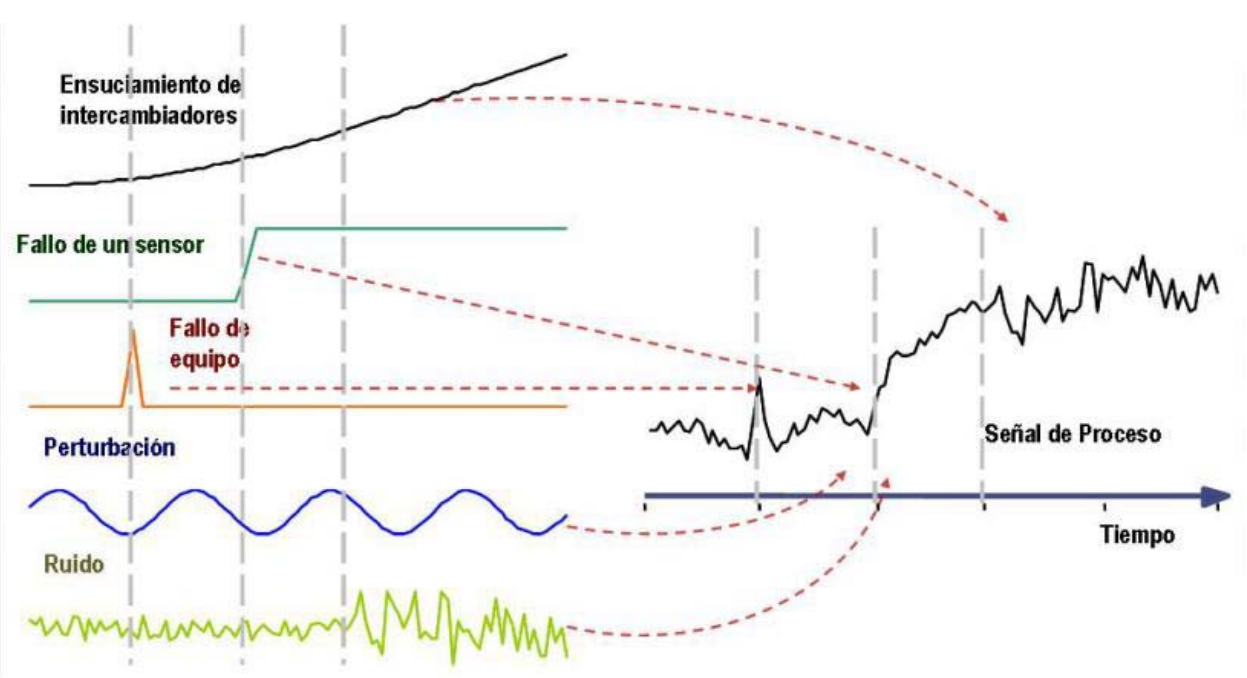

Figura 1.1. Señal de proceso

El campo de la teoría de información provee de técnicas que ayudan al pronóstico, cuantificación de complejidad, detección de irregularidades, identificación de comportamientos, así como clasificación de información en series de tiempo de sistemas dinámicos. La relevancia de la entropía, en este campo, consiste en su fácil interpretación física. Por ejemplo, la entropía propuesta por Shannon (1948) caracteriza la ganancia de información y mide el desorden e incertidumbre existente en una señal dinámica; o la entropía de Kolmogorov-Sinai mide la velocidad exponencial a la cual la información es obtenida en una señal dinámica.

En el presente trabajo de investigación se planteo el uso técnicas de entropía aproximada $(A p E n)$ durante la acción de monitoreo de procesos como columnas de destilación y procesos de corrosión electroquímica. El objetivo fue identificar inestabilidades o irregularidades como consecuencia de perturbaciones, fallas de operación, formación de picaduras, pasivación o pérdida de metal. Los análisis de entropía consistieron en evaluar las fluctuaciones y/o regularidades en las series de tiempo de los procesos analizados. La variabilidad de los sistemas dinámicos analizados se cuantificaron de acuerdo a valores de entropía, donde valores altos corresponden a señales complejas.

Se propuso el análisis de entropía aproximada sin tendencia $(D E A)$, el cual es una modificación de la entropía aproximada $(A p E n)$ propuesta por Pincus (1991). El análisis de 
Introducción

$D E A$ considera que los sistemas dinámicos bajo estudio pueden ser afectados por dinámicas no estacionarias que afectan su tendencia en las series de tiempo. Además se empleó el análisis de entropía multiescala $(M E A)$ para cuantificar la complejidad de señales dinámicas en un rango definido de escalas. El análisis de $M E A$ se basa en la suposición de que los sistemas dinámicos usualmente presentan dinámicas aleatorias que están lejos de la perfecta regularidad. Por consiguiente, en su lugar se presentan dinámicas complejas que normalmente revelan su estructura en múltiples escalas de espacio y tiempo.

\subsubsection{Monitoreo de procesos químicos}

El esquema de monitoreo se ha abordado fundamentalmente desde tres perspectivas distintas: métodos basados en conocimiento, analíticos y señales. Es importante señalar que estos métodos no plantean soluciones competitivas sino complementarias. Algunos ejemplos del monitoreo de procesos basados en conocimiento son los sistemas expertos, redes neuronales y los basados en la lógica difusa. Garces-Rosas (1999) plantea un método de detección y diagnóstico de fallas de dos etapas, basado en el conocimiento, donde a través de un indicador permite determinar la existencia de anomalías en el proceso y luego, a partir de la generación de un patrón se define el origen de dicho desajuste. Sin embargo, el inconveniente es que si no existen suficientes datos de fallas, el modelo no puede aprender a manejar el problema.

En cambio, los métodos analíticos emplean un modelo del proceso para identificar fallas que pueden conducir a inestabilidades (Pugjaner y col., 2006). Por ejemplo, Tarantino (1999) a partir del modelo de un sistema dinámico lineal variante en el tiempo, diseñó filtros y observadores para la detección de fallas. O bien, Sánchez (2002) utilizó la técnica de estructura de transición robusta para los sistemas con retraso de tiempo para generar modelos para representar las posibles anormalidades que se podrían presentar en el proceso. Sin embargo, aunque los sistemas lineales son bien establecidos, el modelado de sistemas no lineales es usualmente complicado.

Por otra parte, en el monitoreo de procesos basados en señales se emplean datos de 
variables físicas involucradas en el proceso como temperatura, velocidad de flujo, presión, voltaje, corriente, entre otras; las cuales pueden ser medidas fácilmente a través de sensores. El monitoreo basado en señales abarca los procesos de control estadístico $(S P C)$ cuyo objetivo principal es utilizar técnicas estadísticas para determinar si la operación de un proceso es normal o anormal a través de gráficos de control. Estos gráficos consisten de una línea central correspondiente a la calidad promedio a la cual debería funcionar el proceso cuando se encuentra presente el control estadístico y dos límites de control, denominados límites de control superior e inferior $(L C S, L C I)$.

La metodología del $S P C$ se basa en la idea fundamental de que procesos de operación normales pueden ser caracterizados por variaciones aleatorias alrededor de un valor medio. Si esta situación ocurre se dice que el proceso está en un estado de control estadístico, o bajo control, y las mediciones en el gráfico de control tienden a estar normalmente distribuidas alrededor de un valor medio. De tal forma que se generan gráficos de control y umbrales de operación para cada variable de manera independiente (Desborough y Harris, 1993; Harris y col., 1999). Por el contrario, si se presentan violaciones en el gráfico de control podría indicar un comportamiento anormal en el proceso o bien una situación fuera de control Seaborg y col., 2004).

El gráfico de control, propuesto por Shewhart (1926) es todavía uno de los mas empleados en la industria (Stoumbos y col. 2000). También se encuentran los gráficos de suma acumulativa (CUSUM) (Page, 1954, Barnard, 1959), promedio móvil ponderado exponencialmente (EWMA) (Roberts, 1959), y los gráficos de control de promedios móviles.

Los gráficos Shewhart son adecuados cuando ocurren cambios considerables y que, por lo tanto, son fácilmente detectables en un diagrama de control. Sin embargo, la mayor desventaja de estos gráficos es su insensibilidad a cambios pequeños en el proceso (frecuentemente $<1.5 \sigma$ ), ya que solo emplean la información de observaciones actuales. Por otra parte, si se requiere detectar cambios pequeños en etapas tempranas se cuenta con alternativas como son los gráficos de control de suma acumulativa (CUSUM) y de promedio móvil ponderado exponencialmente $(E W M A)$. Estos gráficos son más sensibles al 
Introducción

movimiento de los pequeños cambios sostenidos en la media del proceso ya que incorporan tanto las observaciones actuales como las del pasado reciente. En el caso del gráfico de control de promedios móviles tiene una sensibilidad intermedia entre los gráficos de control de Shewhart y de EWMA o CUSUM para detectar pequeños cambios graduales en la media del proceso.

En los gráficos de $S P C$ se estima que las propiedades estadísticas como la media o varianza de las variables medidas son repetibles para cualquier condición de operación. No obstante estos métodos ignoran los efectos de interdependencia entre variables, correlación espacial. Además se desprecian las relaciones de una variable medida en distintos instantes de tiempos, correlación temporal. Debido a estas razones estás técnicas son muy poco robustas en la detección de fallas.

Se han desarrollado diversas investigaciones en el área de monitoreo en columnas de destilación que comprenden las tareas de adquisición, análisis y presentación de datos mediante interfaces gráficas (Zamarreño y Rueda, 2003; Rodriguez, 2005).

\subsubsection{Teoría de información}

Los sistemas de control no siempre compensan satisfactoriamente los efectos de perturbaciones, fallas en sensores, actuadores y en componentes, lo que resulta en inestabilidades en los procesos (Bakshi, 1998). Una característica particular de las inestabilidades dinámicas es el comportamiento no lineal y no estacional en las series de datos de variables involucradas en los procesos (Vela-Martínez y col., 2009). Por lo que, el problema de extraer información útil de este tipo de datos se incrementa cuando además presentan ruido que es inherente a la operación del proceso, ya sea de naturaleza eléctrica, térmica, o bien por tiempos muertos o debido a la transmisión de señales.

El concepto de entropía, en teoría de información, es un concepto poderoso para caracterizar la diversidad de patrones contenidos en una serie de tiempo, el cual surge de la teoría matemática de la comunicación (Shannon, 1948). Donde la entropía puede verse como una medida de información que se transmite en un sistema (Ecuación 1.1). De esta forma, 
Introducción

cuanto mayor es la entropía, más compleja es la dinámica subyacente del sistema.

$$
H(P)=-\sum_{i=1}^{n} p(i) \ln p(i)
$$

El procesamiento de señales se realiza de distintas formas con el objetivo de encontrar características que puedan proveer de información importante en un proceso. Por ejemplo, la transformada rápida de Fourier $(F F T)$ es la base de muchas operaciones fundamentales en el procesamiento de señales. Es comúnmente aplicada para monitorear sistemas en la industria. Sin embargo, la FFT no es adecuada para analizar fallas presentes en la instrumentación de un proceso ya que se presentan características no estacionarias (fenómenos oscilantes localizados en el tiempo), transitorios y/o cambios abruptos que exhiben patrones de frecuencia variables en el tiempo (Guillen y col., 2005). Además, se pierde información temporal relacionada a la localización en el tiempo de la falla. Esto es, el método sólo es adecuado para señales lineales y estacionarias, con resultados aceptables en la práctica en una gran variedad de procesos (Jáuregui-Correa y Gonzalez-Brambila, 2010).

Por otra parte, los conceptos de entropía tienen ventaja sobre los métodos tradicionales de procesamiento de señales, ya que exhiben la estructura intrínseca de una serie de tiempo de un sistema, debido a que consideran estadísticamente la diversidad de patrones en un sistema. Por ejemplo, los conceptos de entropía de Shannon y Kolmogorov-Sinai caracterizan la ganancia de información, proporcionando un índice de desorden e incertidumbre. En particular, la entropía de Kolmogorov-Sinai indica el ritmo exponencial en el cual la información se obtiene cuando el sistema está dinámicamente perturbado.

En el campo de la Ingeniería Química, el análisis de entropía de Shannon desde la última década ha sido ampliamente empleado en el área de mecánica de fluidos. Esto es, la entropía de Shannon ha sido utilizada en la caracterización de comportamientos dinámicos de flujos multifásicos (Zhang, 1991; Zhang y Shi, 1999; Shi y col., 2000; Zhong y Zhang, 2005; Zhong y col. 2009: Yurusoy y col., 2006), cuantificación de la calidad de mezcla en diferentes sistemas Camesasca y col., 2006; Chang y Yang, 2006) y en la descripción de inestabilidades de ondas de densidad de flujo de dos fases (Shi y col., 2004). 
Por otra parte, en el área de control de procesos se ha empezado a emplear el concepto de entropía de Shannon en la detección e identificación de posibles fallas (Yan y Gao, 2007) y en la localización de sensores para la detección de fallas (Orantes y col., 2006, 2007, 2008). Por ejemplo, Orantes y col. (2006) proponen una metodología basada en técnicas de clasificación de la cantidad de información que se mide por el concepto de entropía Shannon, a fin de abordar el problema de la ubicación de sensores en la identificación de fallas en un reactor continuo de intensificación de placa abierta. Los autores definen un indicador de ganancia de información, donde un valor máximo de entropía corresponde al mínimo de información (total incertidumbre) y la entropía más pequeña equivale a la mayor información en la detección de fallas. En estudios posteriores Orantes y col. (2007, 2008) demostraron la utilidad de la metodología de localización de sensores para detectar fallas basada en la entropía de Shannon en procesos donde no se cuenta con un modelo matemático o estructural, o bien el modelo matemático es demasiado complejo.

Sin embargo, un inconveniente de los conceptos tradicionales de entropía es la necesidad de una serie infinita de datos, libres de ruido con una precisión y resolución infinitas (Dorfman, 1999). Esto es una desventaja ya que las mediciones de los sistemas reales son muestreadas con limitada resolución $(\varepsilon)$ y finita velocidad de muestreo $\left(1 / T_{S}\right)$, lo que conduce a series de datos finitos.

Para resolver el problema en el cálculo de la entropía de Shannon, Pincus (1995) estableció el empleo de la entropía aproximada ( $A p E n$, por sus siglas en ingles) como un índice de irregularidad y complejidad relacionada con la aleatoriedad en series de datos. Las ventajas en el cálculo de $A p E n$ es que puede manejar datos no lineales y no estacionarios, requiere un número relativamente pequeño de número de observaciones, y además puede ser usado en señales ruidosas.

Este nuevo concepto de $A p E n$ ha sido ampliamente estudiado e investigado en campos como biomédica (Goldberger y col., 2002, Hornero y col., 2005; Hassan y col., 2011), fisiológicas (Richman y Moorman, 2000), finanzas (Pincus y Kalman, 2004, Pincus, 2008; Alvarez-Ramirez y Rodriguez, 2011) y mecánica (Yan y Gao, 2007; Pérez-Canales y col. 
Introducción

2011). Donde se ha demostrado que el análisis de $A p E n$ es capaz de discriminar la información de las señales en estudio, mientras que estadísticos clásicos como la media o desviación estándar no muestran una clara distinción entre los casos de estudio.

El cálculo de $A p E n$ se basada en la probabilidad de que conjuntos de patrones en las series de tiempo, que son similares, continúan siendo semejantes en el próximo incremento de comparación. De tal manera que, valores bajos de ApEn reflejan series de tiempo con mayor regularidad y valores altos se asocian a series de tiempo con menos predictibilidad, más complejas, dentro de la escala de tiempo $(\tau)$ (Pincus y Singer, 1995).

El estadístico de ApEn potencialmente puede distinguir entre una gran variedad de sistemas determinísticos de baja dimensión, periódicos y múltiples periódicos, caóticos de alta dimensión, estocásticos y mezclados (estocásticos y determinísticos) (Pincus, 1991).

Pincus (1991) exploró los efectos de la tolerancia $(\varepsilon)$, longitud de datos $(N)$ y el vector de dimensión $(m)$ en el desempeño del cálculo computacional de $A p E n$, donde se encontraron estadísticos estables para $N>1000$. Donde además, se propuso el uso de $m=2$ y $\varepsilon=0.15 \sigma$, donde $\sigma$ es la desviación estándar de la serie de tiempo original.

Los sistemas dinámicos muestran patrones complejos temporales a través de un amplio rango de escalas de tiempo. Es decir, una serie de tiempo puede ser más regular en una escala de tiempo y más irregular en otra. Es por ello que, Costa y col. (2002) introdujeron el estadístico entropía multiescala ( $M S E$, por sus siglas en ingles) con el fin de cuantificar la complejidad de series de tiempo sintéticas y reales en diferentes escalas de tiempo. Este concepto de $M S E$ se basa en la ApEn propuesta por Pincus (1991). Costa y col. (2002) reportan que la $M S E$ es capaz de discriminar entre tipos de comportamientos de señales biológicas. Los autores también realizan un análisis de $M S E$ de personas sanas, jóvenes y ancianos, encontrando que la entropía para las personas jóvenes siempre es mayor que el de las personas ancianas para todas las escalas de tiempo. Concluyen que la edad es un factor importante en la eficiencia del sistema de control cardíaco, y que conforme envejecemos perdemos complejidad. 


\section{INTRODUCCIÓN}

\subsection{Objetivos}

\subsubsection{Objetivo general}

Emplear análisis de entropía aproximada $(A p E n)$ como herramienta durante el monitoreo de columnas de destilación y sistemas de corrosión electroquímica. Con el propósito de identificar inestabilidades o irregularidades como consecuencia de perturbaciones, fallas de operación, formaciones de picaduras, pasivación o pérdida de metal en los procesos analizados.

\subsubsection{Objetivos particulares}

Como parte del objetivo general de la tesis se plantean los siguientes objetivos particulares que ayudan a complementar la investigación doctoral.

- Analizar señales temporales sintéticas del tipo $1 / f^{\beta}$ con el fin de evaluar los análisis de entropía aproximada propuestos como son el $D E A$ y el $M A E$ en la identificación de fluctuaciones y/o regularidades.

- Emplear análisis de entropía aproximada en las señales de intensidad de difracción de rayos $X$ con ruido intenso. Con el propósito de validar la identificación de patrones 
Introducción

de regularidad de series temporales de procesos que son comunes en la caracterización fases de un material con cierto grado de amorficidad.

- Examinar la complejidad en series temporales de cada etapa en columnas de destilación empleando análisis de entropía aproximada, y de esta forma analizar su distribución de contenido de información.

\subsection{Organización de la tesis}

El Capítulo 1 corresponde a la introducción general de la tesis. En la Sección 1.1 se detallan los antecedentes más relevantes de la investigación doctoral en los campos de monitoreo de procesos químicos en el área de ingeniería química y teoría de información. Posteriormente en la Sección 1.2 se describen los objetivos tanto el general como los específicos de la investigación doctoral.

La tesis se divide principalmente en dos partes. En la Parte I] se evalúan los análisis de entropía en la identificación de fluctuaciones y/o regularidades en series temporales. Las señales que se emplearon en la validación fueron señales sintéticas del tipo $1 / f^{\beta}$ y señales reales de un proceso de difracción de rayos $X$ con ruido intenso. En la Parte II se presentan los resultados obtenidos en el monitoreo de columnas de destilación y de sistemas de corrosión electroquímica mediante análisis de entropía. En cada uno de los capítulos se presenta una breve introducción de los conceptos más importantes, así como de la metodología empleada y los resultados alcanzados. El contenido de cada capítulo se describe a continuación.

En el Capítulo 2 se analizan señales de los ruidos $1 / f^{\beta}$, ya que representan patrones característicos de muchos fenómenos naturales, de tal manera que reflejan la estructura de los sucesos donde se manifiestan. Se analizan este tipo de señales sintéticas con la finalidad de obtener información de variaciones en las señales a distintas escalas de tiempo, y así mostrar el rendimiento de los análisis de entropía en la identificación de fluctuaciones y/o regularidades en series temporales.

En el Capítulo 3 se describe el análisis realizado a señales empleadas en la caracterización 
de fases como son las señales de difracción de rayos $X$ con ruido intenso. El objetivo de este capítulo es demostrar que los análisis de entropía son capaces de obtener información valiosa de señales temporales de fenómenos físicos, aparentemente aleatorias y con ruido intenso, como son las señales de difracción de rayos $X$. Los resultados muestran que el estadístico de ApEn permite obtener información relevante de señales aleatorias y aparentemente carentes de información.

En el Capítulo 4 se presentan las características de las columnas de destilación estudiadas, la metodología, así como los resultados obtenidos en los análisis de entropía para fines de monitoreo en los sistemas de destilación.

En el Capítulo 5 se aborda el análisis de series de tiempo del acero inoxidable sumergido en solución de bicarbonato que se obtienen a partir de la técnica de ruido electroquímico $(E N)$. Los resultados obtenidos en los análisis de entropía muestran que el estadístico de entropía obtiene información acerca del comportamiento del sistema que puede ser asociado con la formación de productos de corrosión, pasivación, re-pasivación del metal o inicio de picaduras. Además los resultados de entropía se contrastan contra los obtenidos con el análisis visual de las señales crudas.

Y finalmente en el Capítulo 6 se presentan las conclusiones generales de la investigación doctoral. 


\section{Parte I}

\section{Análisis de señales sintéticas y de difracción de rayos $X$}


CAPÍTULO 2

SENALES SINTÉTICAS $1 / F^{\beta}$

En este Capítulo 2 se analizaron señales de ruido $1 / f^{\beta}$ ya que presentan patrones característicos asociados a muchos fenómenos naturales como son terremotos, comportamientos en bolsas de valores, distribución de montañas y muchos más. Se realizaron análisis de entropía aproximada sin tendencia $(D E A)$ y a multiescala $(M A E)$ con el objetivo de demostrar que los análisis de entropía son capaces de proveer de información en la identificación de fluctuaciones y/o regularidades en series temporales sintéticas a distintas escalas de tiempo. En la Sección 2.1 se presenta una breve introducción de algunas características generales de las señales de ruido $1 / f^{\beta}$, las cuales abarcan desde el ruido blanco hasta de movimiento browniano. Posteriormente en la Sección 2.2 se explica la metodología empleada en el cálculo computacional de la entropía. Finalmente en la Sección 2.3 se presentan los resultados obtenidos en los análisis de entropía ( $D E A$ y $M A E)$, demostrando de esta forma que dichos análisis son capaces de reconocer o identificar patrones estructurados en las señales analizadas. 


\subsection{Introducción}

Muchos fenómenos físicos exhiben fluctuaciones los cuales pueden ser descritos por medio de una función de frecuencia $f$ que se comporta como $p(f)=1 / f^{\beta}$, donde exhiben características relacionadas con la dinámica de las propiedades de disipación e irreversibilidad de sistemas físicos, debido a que reflejan la estructura de los sucesos donde aparecen (Kuzovlev, 2012). Este tipo de señales han sido empleadas para representar características asociadas a fenómenos naturales (Gardner, 1978; Milotti, 1996; nslij genetics, 2011). Algunos ejemplos de su empleo han sido en la caracterización de patrones de terremotos Wassermann, 2002), sistemas de corrosión (Castañeda y col., 2010), comportamientos en mercados financieros (Valderas y col., 2002), tráfico en redes informáticas (Pugliese y Castellano, 2009), entre otros.

En la función de frecuencia $1 / f^{\beta}$, cuando el valor del exponente es $\beta=0$ se tiene el ruido blanco $\left(1 / f^{0}\right)$ y cuando $\beta=2$ se llama ruido browniano $\left(1 / f^{2}\right)$. En medio existen una gran cantidad de fenómenos que tienen un espectro de frecuencia que esta entre el ruido blanco $\left(1 / f^{0}\right)$ y de movimiento browniano $\left(1 / f^{2}\right)$, de tal forma que se tiene un espectro de frecuencia de la forma $1 / f^{\beta}$ donde $0>\beta<2$, llamados ruidos de centelleo Miramontes, 1999).

El ruido blanco o aleatorio se presenta cuando una señal tiene un comportamiento aleatorio (estocástico), que se caracteriza por el hecho de que sus valores en la señal en dos tiempos diferentes no guardan correlación estadística (Figura 2.1a). Una de las propiedades mas importantes de este tipo de ruido es que el espectro de frecuencias tiene una distribución $1 / f^{0}$. Y como consecuencia, la gráfica de su densidad espectral de potencia ( $P S D$, siglas en inglés de Power Spectral Density) es plana; esto significa que la señal contiene todas las frecuencias y todas ellas muestran la misma potencia.

En el caso del ruido de movimiento browniano o café (Figura 2.1c), tiene la propiedad de que su espectro de frecuencia tiene una distribución $1 / f^{2}$. De tal forma que, cuando la frecuencia vale 1 la amplitud vale $1 / 1^{2}=1$. Cuando la frecuencia vale 2 la amplitud vale $1 / 2^{2}$ o sea $1 / 4$ y así sucesivamente. Por lo que, en el ruido de movimiento browniano las 
componentes con frecuencia mas altas son prácticamente insignificativas Valderas y col. 2002 ).

\subsection{Metodología}

Las señales sintéticas $1 / f^{\beta}$ son señales cuyo comportamiento es ampliamente estudiado. Además de que representan el comportamiento de sistemas físicos, financieros o biológicos muestran características fractales que pueden ser caracterizadas a través de este tipo de señales. Por lo cual, para validar los análisis de entropía se emplearon señales sintéticas de ruido $1 / f^{\beta}$ que abarcan desde el ruido blanco, pasando por señales $1 / f^{\beta}$ donde $0>\beta<2$, hasta el ruido de movimiento browniano, disponibles en la base de datos PhysioNet (2011).

Se realizaron análisis de $D E A$ con el objetivo de revelar información a cerca de fluctuaciones o irregularidades en las series temporales sintéticas $1 / f^{\beta}$. Además, también se llevaron a cabo análisis de $M E A$ para evaluar la complejidad de las señales a distintas escalas de tiempo. En ambos casos, los valores de entropía se interpretan de la siguiente forma: valores bajos reflejan una mayor regularidad en las series temporales, mientras que valores altos están asociados con una menor predictibilidad o mayor complejidad en la señal.

Para el cálculo computacional de la entropía (Apéndice B, C y D se utilizaron datos con una longitud de $N=30000$, el vector de dimensión $m=2$ y la tolerancia $\varepsilon=0.15 \sigma$, donde $\sigma$ es la desviación estándar de la serie de tiempo original. Tal como lo propone Pincus (1991) en el análisis de los parámetros de $A p E n$.

\subsection{Resultados}

En la Figura 2.1 se muestran algunas señales sintéticas $1 / f^{\beta}$ analizadas que abarcan desde el ruido blanco, pasando por señales $1 / f^{\beta}$ donde $0>\beta<2$, hasta la señal de ruido de movimiento browniano.

Los resultados de los análisis de $D E A$ a diferentes escalas de tiempo para las señales sintéticas $1 / f^{\beta}$ se muestran en la Figura 2.2. Obsérvese que para la secuencia de ruido 


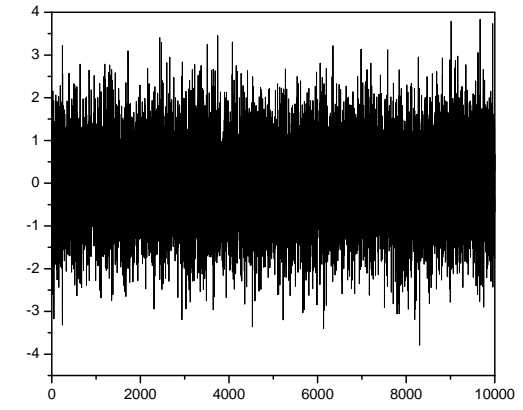

(a) Señal de ruido blanco $(\beta=0)$

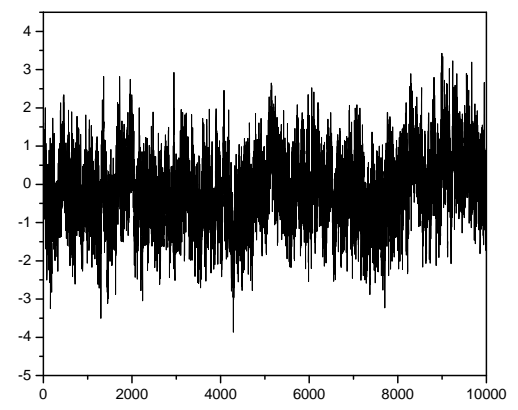

(b) Señal sintética $(\beta=0.8)$

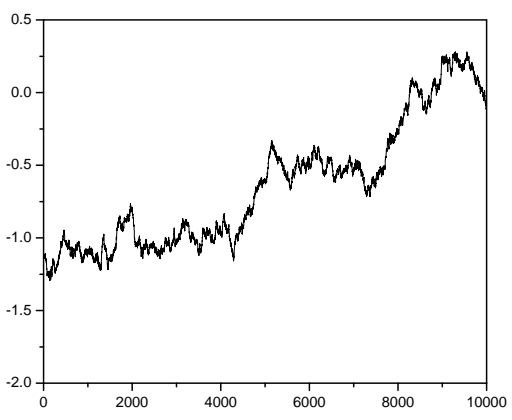

(c) Señal de movimiento browniano $(\beta=2)$

Figura 2.1. Ejemplos de algunas señales sintéticas de ruido $1 / f^{\beta}$.

blanco o aleatorio $\left(1 / f^{0}\right)$ se tienen los mayores valores de entropía a distintas escalas de tiempo, lo cual indica una falta orden o patrón estructurado en cualquier escala. Esto es, el ruido blanco presenta un comportamiento aleatorio de tal forma que el valor de la señal en un instante no tiene ninguna relación con el valor de la señal en el siguiente instante, por lo cual se dice que no tiene memoria o que es anticorrelacionado. En el caso de las señales $1 / f^{\beta}$, cuando $\beta$ se encuentra entre valores que corresponden a $0>\beta<2$, el valor de $D E A(\tau)$ es una función que decae monotónicamente en función de la escala de tiempo. Para el caso del movimiento browniano $(\beta=2)$ se observa una mayor rapidez de disminución de entropía, lo cual significa que a escalas pequeñas, microestructura, se presentan menos patrones estructurados. Mientras que a escalas mayores, macroestructura, se observa una 
disminución en la entropía, ya que presenta mas patrones estructurados, por lo cual se dice que la señal está correlacionada.

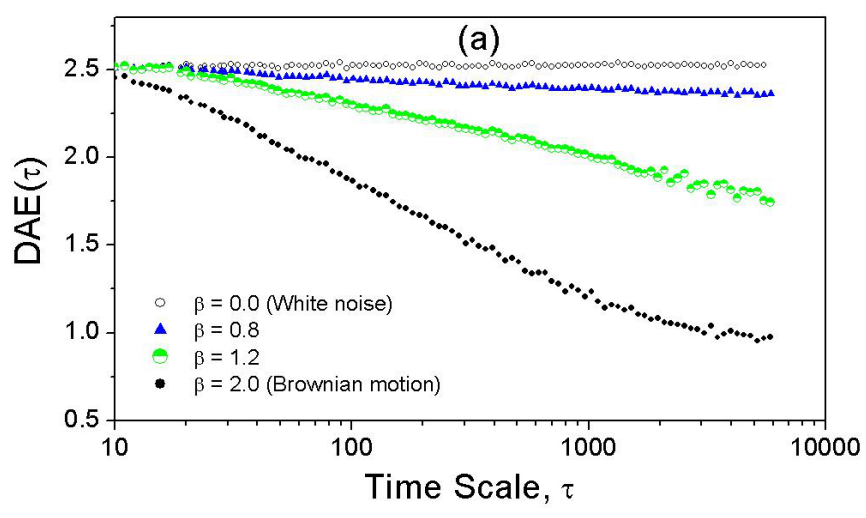

Figura 2.2. $D E A$ para señales $1 / f^{\beta}$

La Figura 2.3 muestra los resultados de los análisis de entropía multiescala ( $M A E$ ) realizado a las señales sintéticas $1 / f^{\beta}$. Donde puede observarse que la señal de ruido blanco mostró los mayores valores de entropía. Mientras que, en la señal de ruido de movimiento browniano se tienen los menores valores de entropía a cualquier escala de tiempo. Los resultados anteriores concuerdan con las conclusiones obtenidas en los análisis de $D E A$, donde se encuentra que la señal de ruido blanco es una señal anticorrelacionada, sin patrones estructurados. En contraste, en la señal de ruido de movimiento browniano se presentan patrones o correlaciones que pueden ser detectadas a través de los análisis de entropía.

Por lo tanto, se demuestra que los métodos de entropía aproximada propuestos en la presente investigación pueden detectar patrones o irregularidades en señales sintéticas que presentan ruido, no son estacionarias y que además presentan tendencia.

Por otra parte, para demostrar que el valor de la entropía disminuye como consecuencia de la estructura intrínseca de la señal, la serie de tiempo de movimiento browniano se barajeo aleatoriamente para destruir correlaciones y, en consecuencia, degradar el contenido de información en la señal de tiempo original. La Figura 2.4 muestra el $D E A$ como una función de la escala de tiempo para la señal de movimiento browniano original $(\beta=2)$ y su 


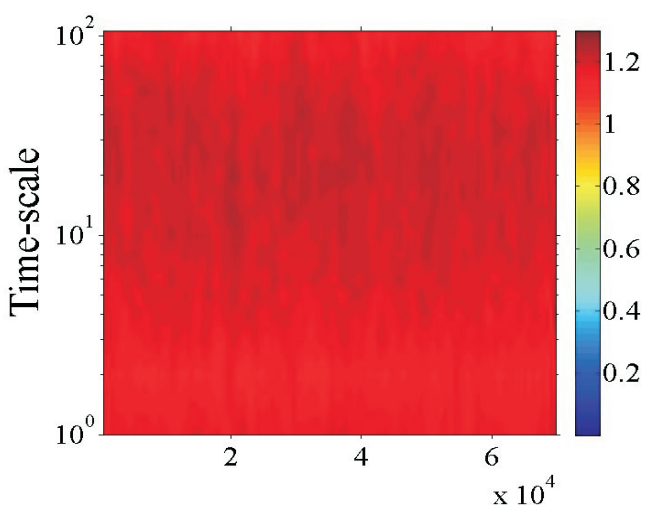

(a) Señal de ruido blanco $(\beta=0)$

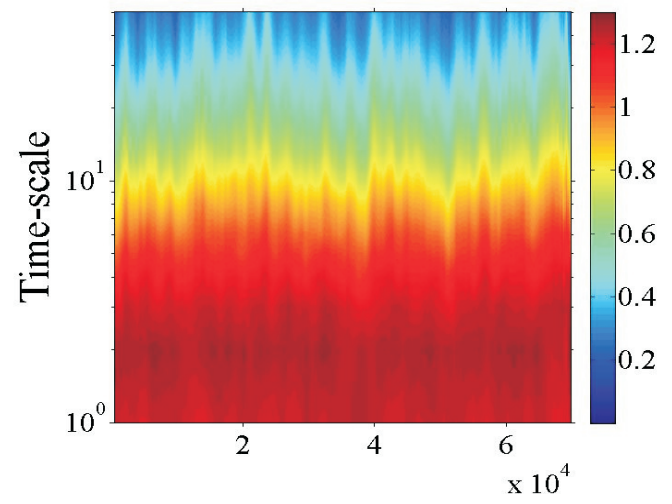

(b) Señal sintética $(\beta=0.8)$

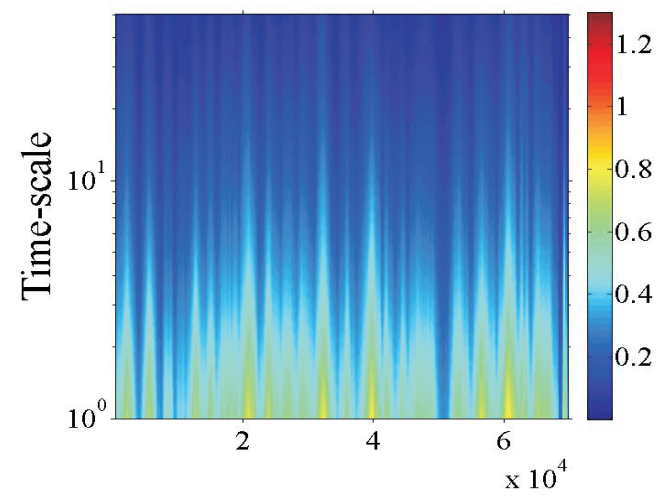

(c) Señal de movimiento browniano $(\beta=2)$

Figura 2.3. Entropía aproximada multiescala de señales sintéticas $1 / f^{\beta}$.

correspondiente señal de tiempo barajeada.

La serie de tiempo barajeada presentó los mayores valores de entropía a diferencia de la señal original. Esto se debe a que durante el barajeo de la señal original se destruyen correlaciones, por consiguiente se tiene una falta de orden o patrones en la estructura subyacente de la señal barajeada. La función de $D E A$ para la señal barajeada es constante para escalas de tiempo > 300, donde además muestra un comportamiento similar al ruido blanco observado en la Figura 2.2. Por otra parte, la señal de movimiento browniano original presenta una pendiente negativa para las distintas escalas de tiempo, lo cual sugiere una contribución de correlaciones o patrones estructurados en la señal. 


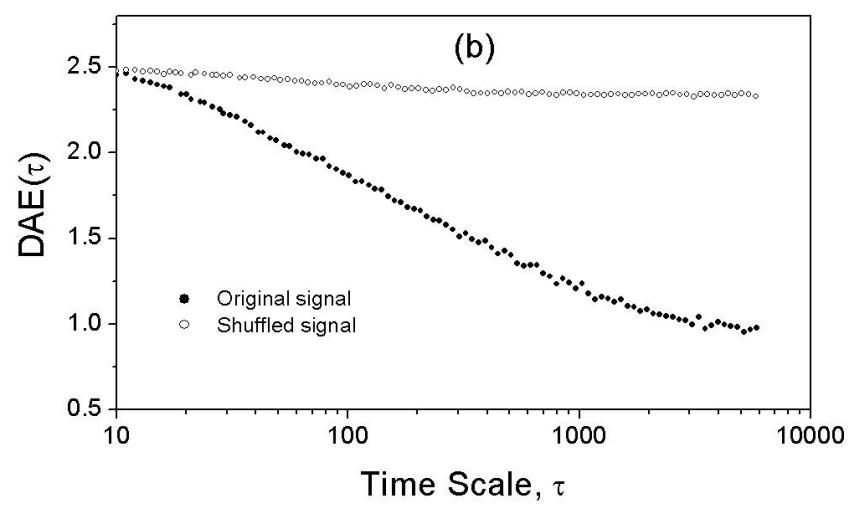

Figura 2.4. $D E A$ para el movimiento Browniano y su señal aleatoria.

Por otra parte, también se planteo la siguiente pregunta: ¿Se podrán detectar perturbaciones en señales temporales a través de análisis de entropía aproximada?. Por lo tanto, se investigó, sí los análisis de entropía propuestos son capaces de detectar perturbaciones en señales temporales sintéticas con una acción de control. Para explorar los efectos de perturbaciones en un sistema de control retroalimentado, se considero el modelo simple de regresión media $x_{i}=x_{r}+K\left(x_{i-1}-x_{r}\right)+w_{i-1}$. Donde $w_{i-1}$ representa una perturbación de ruido blanco, $x_{r}$ es una señal de referencia, $K\left(x_{i-1}-x_{r}\right)$ es la señal de control retroalimentado y $K$ es la ganancia del control retroalimentado. En ausencia de disturbios, la señal $x_{i}$ converge al valor requerido $x_{r}$ para $-1<K<+1$. En la Figura 2.5 a se muestra el comportamiento de $D E A$ para tres valores distintos de ganancia $K$. Donde se observa una reducción de la entropía para escalas de tiempo altas, lo cual está relacionado con la acción de control que ejerce la ganancia en el control retroalimentado.

Además se consideró que, no solamente las perturbaciones en un proceso afectan la acción de control del mismo; también las acciones de retraso en los controladores retroalimentados pueden limitar seriamente la capacidad de un sistema para reducir efectos adversos de disturbios (Morari y E. 1989). Se exploró el comportamiento de las señales temporales bajo condiciones de retrasos en el control retroalimentado a través de la entropía aproximada. 
Para lo cual, se consideró el modelo de control $K\left(x_{i-d}-x_{r}\right)$, donde $d>1$ representa el retraso. La Figura 2.5b muestra el comportamiento de $D E A$ para $K=0.5$ y $d=6$. Se observa que el retraso en la retroalimentación es incapaz de reducir la incertidumbre para escalas de tiempo altas. De esta forma, el sistema es operado en condiciones inestables donde el valor deseable no puede alcanzarse. Finalmente, es interesante observar que la curva de $D E A$ para el caso de control retroalimentado con retrasos se comporta de manera similar a la del ruido blanco. Esto sugiere que la acción de control retroalimentado con retrasos representa una condición de control deteriorado.

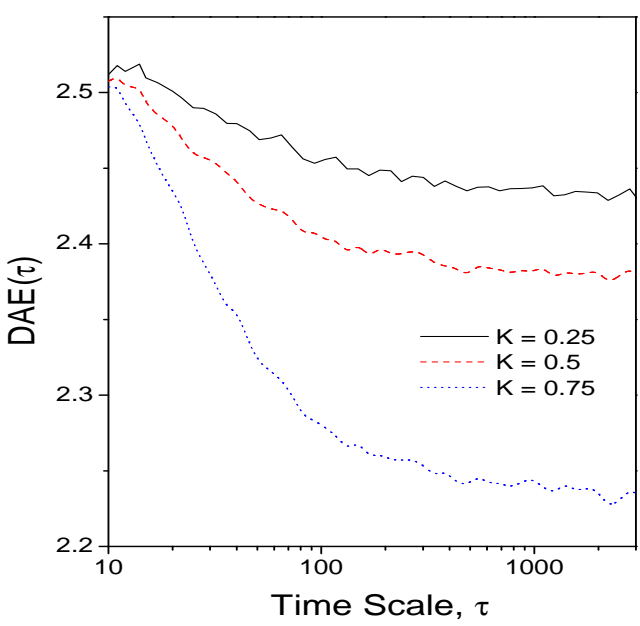

(a)

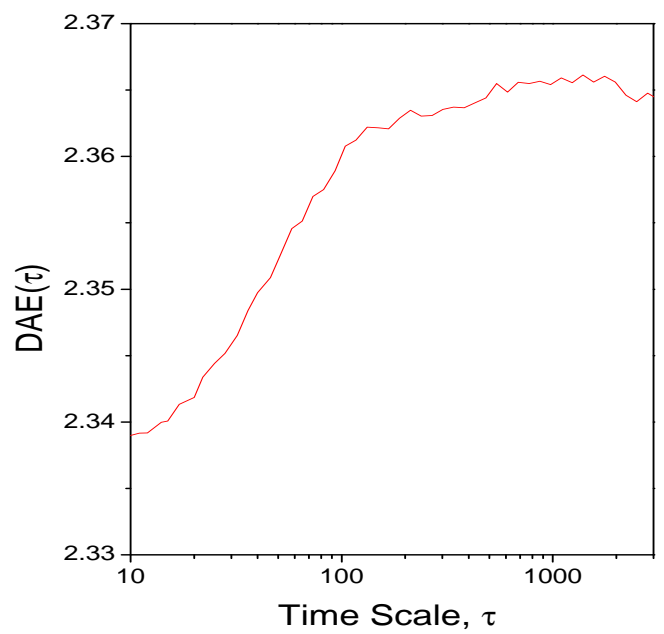

(b)

Figura 2.5. DEA para la señal de ruido blanco con acciones de control por (a) retroalimentación con amortiguación y (b) con retardos.

\subsection{Conclusiones}

Se evaluó el rendimiento de los análisis de entropía a través de señales sintéticas $1 / f^{\beta}$, cuyo comportamiento es conocido. Se utilizaron este tipo de señales debido a que muchos sistemas que presentan características fractales pueden ser caracterizadas a través de estas.

Los resultados de los análisis de $D E A$ y $M A E$ de la señal de ruido blanco $\left(1 / f^{0}\right)$ 
muestran que la señal presenta valores altos de entropía, lo cual indica una falta de orden o patrones estructurados en la señal en cualquier escala de tiempo. Lo anterior concuerda con el comportamiento aleatorio o anticorrelacionado del ruido blanco. En el caso de la señal de ruido browniano $\left(1 / f^{2}\right)$ presenta una tendencia negativa en los valores de entropía a escalas mayores de tiempo, de tal forma que se dice que la señal está correlacionada. Lo anterior coincide con el comportamiento descrito por este tipo de señal. Por otra parte, los resultados del análisis realizado a la señal de movimiento browniano barajeada muestran que la señal barajeada presentó valores altos de entropía. Esto es, debido a que durante el barajeo de la señal original se destruyen correlaciones existentes y por lo cual se destruyen patrones estructurados existentes en la señal original.

Además se exploraron los efectos de un sistema de control retroalimentado, donde se observa una reducción de la entropía para escalas de tiempo altas, lo cual está relacionado con la acción de control que ejerce la ganancia en el control retroalimentado. También se examino el comportamiento de entropía bajo condiciones de retrasos en el control retroalimentado. Los resultados muestran que el retraso en la retroalimentación es incapaz de reducir la incertidumbre para escalas de tiempo altas. De esta forma, el sistema es operado en condiciones inestables donde el valor deseable no puede alcanzarse.

Los resultados anteriores demuestran que los análisis de entropía ( $D E A$ y $M A E$ ) propuestos en la investigación pueden detectar correlaciones o patrones estructurados en series temporales considerando efectos de tendencia a diferentes escalas de tiempo. 


\section{CAPÍTULO 3 \\ LSEÑALS DE INTENSIDAD DE DIFRACCIÓN DE RAYOS $X$}

En este Capítulo 3 se empleó el análisis de entropía aproximada multiescala para caracterizar los patrones de intensidad de difracción de rayos $X$ con ruido intenso. En la Sección 3.1 se presenta una introducción en el uso de este tipo de señales en la caracterización de materiales con cierto grado de amorficidad, donde la identificación de picos están asociados a ciertas fases del material. En la Sección 3.2 se presenta la metodología empleada en el análisis de entropía aproximada multiescala $(M A E)$. Para ilustrar la aplicabilidad del método se utilizaron como casos de estudio distintas muestras de alúmina calcinada a diferentes temperaturas, debido a la gran información existente sobre la estabilidad de fases y transiciones. Finalmente en la Sección 3.3 se muestran los resultados obtenidos en el análisis de entropía aproximada multiescala $(M A E)$. Donde se demostró que el análisis de entropía es capaz de detectar regiones angulares en las señales de intensidad asociadas a fases de alúmina correspondientes a fichas de difracción de polvos $(P D F)$.

\subsection{Introducción}

La difracción de rayos $X$ en polvos $(X R D)$ es tal vez la técnica con mayor aceptación y confiabilidad en la caracterización de materiales. En general, la muestra a estudiar debe 
3. Señales de intensidad de difracción de rayos $X$

encontrarse en forma de granos finos del material cristalino siendo el comportamiento de los patrones de $X R D$ consecuencia de la simetría y orientación de los cristales en la muestra de estudio. Por lo tanto, cuando los patrones de difracción $2-D$ son obtenidos estos muestran aros concéntricos de picos dispersos correspondientes a diferentes espacios-d en la estructura cristalina. Donde la posición e intensidad de los picos se emplean para identificar la estructura o fase principal del material.

Una ventaja adicional de la XRD implica una preparación no complicada del material y una caracterización no destructiva del mismo, lo cual es conveniente en la caracterización de materiales desconocidos. La caracterización de materiales a partir de datos de $X R D$ se consigue por comparación de los patrones de difracción contra un estándar conocido o con los datos proporcionados por The International Center for Diffraction Data.

Los sólidos cristalinos exhiben patrones de difracción distintivos con fuertes picos de intensidad que son fácilmente observados. Ambos, la posición que corresponde a la configuración de espacios y la intensidad relativa de las líneas son indicadores de una fase particular del material, los cuales se emplean como patrones de comparación. En contraste a los sólidos cristalinos donde los patrones de $X R D$ exhiben marcados picos, los materiales no cristalinos pueden conducir a señales con ruido intenso. Como ejemplos de materiales con algún grado de amorficidad están varios polímeros y semiconductores, donde la cristalinidad está limitada a una parte de la estructura molecular. Un calcinado incompleto a bajas temperaturas de materiales cerámicos puede producir estructuras amorfas donde las fases cristalinas pueden estar mezcladas con material no cristalino dando configuraciones geométricas complejas con patrones no regulares.

Las Figuras 3.1. a y 3.1.b muestran patrones de difracción de rayos $X$ correspondientes a las temperaturas de calcinación de 200 y $500^{\circ} \mathrm{C}$ para alúmina preparada por el método sol-gel (Wang y col., 1999). De acuerdo a la ruta de transformación de fases más probable para las condiciones de preparación de la alúmina (Gates y col., 1979), la señal de intensidad correspondiente a $200^{\circ} \mathrm{C}$ muestra tres picos prominentes que sugieren la presencia de $\gamma^{*}$ bohemita a $\sim 10$ grados (Wang y col., 1999), grupos de óxidos a $\sim 22$ grados (Liu y col., 
2008) y bayerita a $\sim 40$ grados (PDF Card $20-0011)$. A pesar de la presencia de estos picos, los patrones de $X R D$ presentan ruido intenso para los cuales las regiones angulares de intensidad regular no pueden ser fácilmente distinguidas.
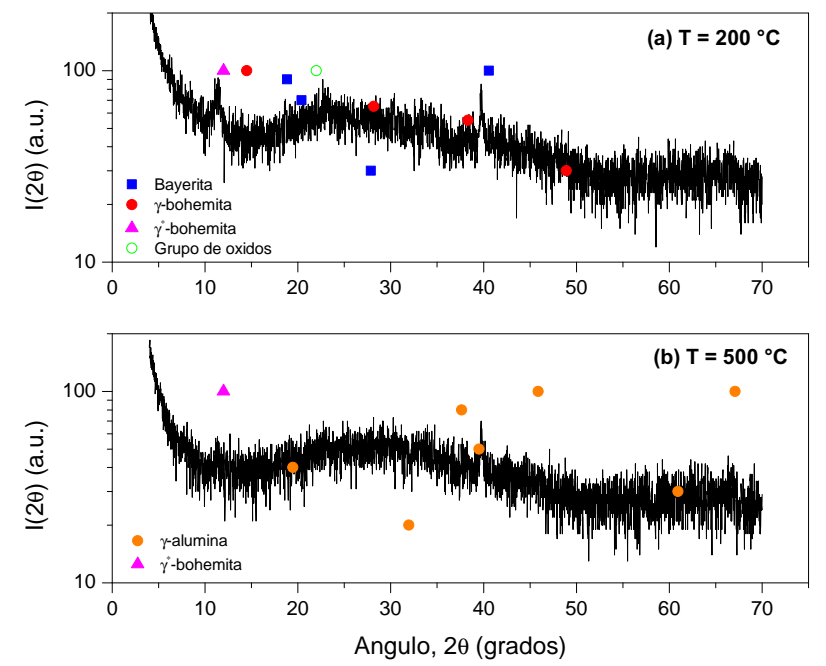

Figura 3.1. Intensidad de patrones $I(2 \theta)$ de rayos $X$ para alúmina calcinada a 200 y $500{ }^{\circ} \mathrm{C}$.

Para el caso de la temperatura de calcinación a $500^{\circ} \mathrm{C}$ se presenta un análisis de mayor reto donde sólo un pico prominente puede observarse. Para estas condiciones de calcinación, los grupos de bayerita y bohemita se convierten en las fases $\eta-$ y $\gamma$-alúmina. El único pico prominente a $\sim 39.5$ grados (50\% de intensidad, PDF Card $10-0425)$ indica la presencia de $\gamma$-alúmina. Sin embargo, otro importante pico de $\gamma$-alúmina (por ejemplo, la línea de intensidad de $100 \%$ a 45.9 grados) no se muestra en los patrones de XRD. En general, los patrones de ruido en estas señales de $X R D$ reflejan la presencia de estructuras espaciales desordenadas. Como consecuencia, la interpretación de los patrones de XRD llega a ser, en muchos casos, un reto para los ojos inexpertos o profesionales no especializados.

Avances continuos en hardware y software, en particular mejoras ópticas y detectores rápidos, han mejorado drásticamente la capacidad analítica de los análisis de difracción de rayos $X$ (por ejemplo, la velocidad de los análisis). Sin embargo, la técnica aún requiere de futuras mejoras para refinar e interpretar los patrones de $X R D$ con ruido intenso como 
3. Señales de intensidad de difracción de rayos $X$

aquellos mostrados en la Figura 3.1. Avances en estos temas pueden permitir a los usuarios de $X R D$ una mayor precisión en la caracterización del material y sus fases.

En este capítulo se propone el enfoque del análisis de entropía aproximada para analizar los patrones de $X R D$ con ruido intenso. Esta técnica ha sido empleada en diversas disciplinas desde economía (Pincus, 2008; Martina y col., 2011; Alvarez-Ramirez y Rodriguez, 2011), ciencias biomédicas (Pincus, 2006; Pan y col., 2011; Hassan y col., 2011) hasta ciencia de materiales (Yan y Gao, 2007; Pérez-Canales y col., 2011) con éxito en la caracterización de señales complejas.

La motivación radica en que el ruido contenido en la señal puede proporcionar información de las fases del material. Es decir, las señales no son aleatorias del todo. Existen regularidades, menores fluctuaciones en la señal que pueden detectarse con el análisis de entropía aproximada sugiriendo la existencia de estructuras más ordenadas, es decir fases cristalinas que pueden ser detectadas en las señales de intensidad. De esta forma, un fuerte pico de intensidad en un ángulo dado se manifiesta porque la radiación dispersa de rayos $X$ se concentra en el detector por geometrías regulares que corresponden a estructuras cristalinas. En contraste, para materiales amorfos no se consigue concentrar la radiación, debido a la ausencia de regularidad.

La idea fundamental es que el patrón de ruido intenso no es tan complejo del todo, sino que contiene patrones internos que pueden asociarse a la estructura del material. Estos patrones pueden ser comparados con fichas de difracción de polvos $(P D F)$ para concluir la presencia o no de probables fases del material. Se seleccionaron como casos de estudio alúmina calcinada a diferentes temperaturas por la gran cantidad de información con respecto a la estabilidad de fases y transiciones disponibles en la literatura científica.

\subsection{Metodología}

La metodología usa el análisis de entropía aproximada multiescala para detectar regiones angulares en los patrones de $X R D$ con ruido intenso, $I(2 \theta)$, que contienen patrones asociados a la regularidad en la estructura del material. La idea fundamental es que patrones 
3. Señales de intensidad de difracción de rayos $X$

regulares de $I(2 \theta)$ reflejan un cierto grado de orden en la geometría del material. En esta forma, un fuerte pico en el patrón de intensidad $I(2 \theta)$ que refleja estructuras de un material altamente organizado corresponde a patrones con mayor regularidad asociados a una menor complejidad. Por otra parte, geometrías altamente desorganizadas corresponden a un comportamiento aleatorio en las fluctuaciones de intensidad de la difracción de rayos $X$.

Basado en estas ideas, la metodología propuesta para analizar los patrones de $X R D$ con ruido intenso consiste en transformar la señal de intensidad $I(2 \theta)$ en un patrón de entropía $E(2 \theta)$ mediante el análisis de entropía aproximada multiescala; es decir,

$$
I(2 \theta) \rightarrow E(2 \theta)
$$

De este manera, el análisis para caracterizar la presencia de fases en el material se llevó a cabo con los patrones de entropía $E(2 \theta)$ en lugar de la señal de intensidad $I(2 \theta)$. Así, bajos valores de $E(2 \theta)$ están asociados con estructuras más regulares, cristalinas, y posibles fases el material. Mientras que altos valores de $E(2 \theta)$ se asocian con estructuras menos regulares, amorfas.

\subsection{Resultados}

Para cada corrida, los datos experimentales de XRD consistieron de 3301 observaciones. El tamaño de la ventana deslizante fue seleccionado como 250 observaciones, fijándose 21 escalas para realizar el análisis.

La Figura 3.2, a muestra la intensidad $I(2 \theta)$ de patrones de $X R D$ para la temperatura de calcinación de $200^{\circ} \mathrm{C}$. Para esta temperatura de calcinación, la alúmina está compuesta probablemente por bayerita y ciertas formas de bohemita, como $\gamma^{*}$-bohemita que tiene la misma estructura cristalina que la $\gamma$-alúmina (Wang y col., 1999). La figura 3.2. a también muestra la localización, extraídas de tarjetas PDF, para bayerita (Card 20-0011) y bohemita (Card $21-1307$ ), indicando que los picos a $\sim 40$ grados pueden ser relacionados a bayerita.

Siguiendo el estudio realizado por Wang y col. (1999), los picos a $\sim 10$ grados pueden ser 
3. Señales de intensidad de difracción de rayos $X$

relacionados a la presencia de $\gamma^{*}$-bohemita. Se ha sugerido que los picos a $\sim 22$ grados pueden estar relacionados a la presencia de grupos de óxidos complejos (Liu y col., 2008). A pesar de que los fuertes picos en los patrones de XRD pueden ser explicados por la presencia de algunas fases de alúmina, la localización de otras fases probables, como bayerita, es incierta debido al intenso ruido que afecta la interpretación de los resultados experimentales. La Figura 3.2.b muestra los correspondientes patrones de entropía $E(2 \theta)$ obtenidos por el análisis de entropía aproximada multiescala. Las siguientes características pueden ser observadas:

1. Se planteó la hipótesis de que fuertes picos de intensidad deberían corresponder a mayores regularidades, menor complejidad en la estructura del material, en términos del análisis de entropía aproximada multiescala, $E(2 \theta)$. Los resultados en la Figura 3.2. b respaldan esta idea mostrando que la intensidad marcada en los picos $\sim 10,22$ y 40 grados corresponden a los menores valores de entropía y por lo tanto a una menor complejidad en la estructura del material que pueden asociarse a fases cristalinas.

2. El patrón de entropía $E(2 \theta)$ no muestra marcados picos como en los patrones de intensidad $I(2 \theta)$ de $X R D$. En lugar, el comportamiento del patrón $E(2 \theta)$ obtenido del análisis de entropía multiescala muestra zonas de mínimos y máximos. Esto sugiere que las fases cristalinas en la estructura de la alúmina no se encuentran aisladas sino que coexisten en una distribución geométrica compleja, reflejada en el ruido intenso de los patrones de intensidad $I(2 \theta)$ de $X R D$. Por ejemplo, la región angular en la cercanía de los 20 grados sugiere un patrón de cristalinidad conformado por bayerita y grupos óxidos. Similarmente, en la cercanía de los 40 grados pueden ser relacionada a un patrón de cristalinidad complejo conformado por bayerita y bohemita.

3. El análisis de entropía aproximada multiescala puede revelar la existencia de regiones asociadas a regularidades no triviales que no son exhibidas por los patrones originales de $X R D$. De hecho, el análisis de entropía aproximada multiescala reveló la existencia de patrones regulares en la cercanía de los 50 grados, con valores de entropía similar 
3. Señales de intensidad de difracción de rayos $X$

a los patrones en la cercanía de los 40 grados. En contraste al patrón de intensidad original $I(2 \theta)$, el análisis de entropía aproximada multiescala sugiere que la región de 50 grados puede estar relacionado a la fase de bohemita.

4. El menor valor de entropía está localizado en la cercanía de los 10 grados, lo cual puede ser atribuido a la presencia de la fase $\gamma^{*}$-bohemita como ya se ha sugerido por Wang y col. (1999).
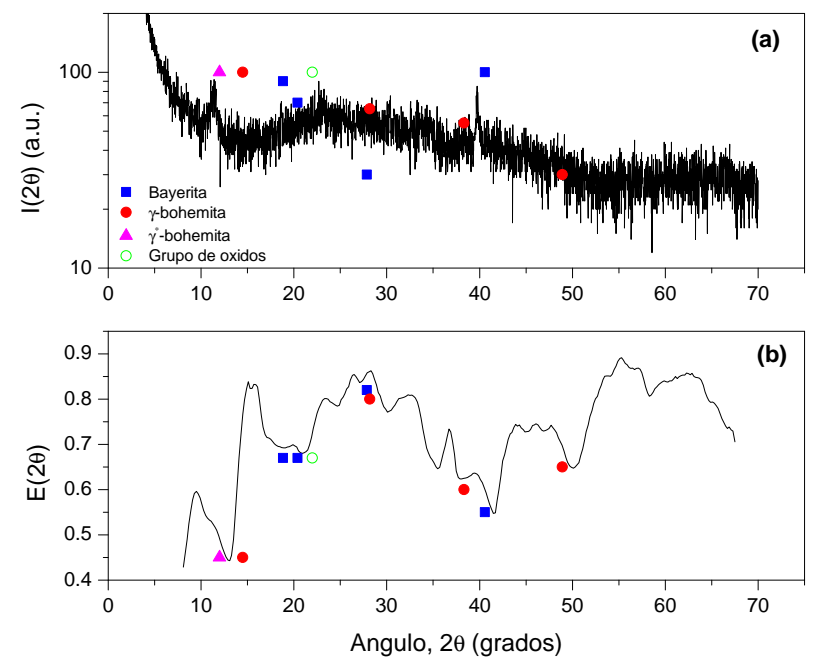

Figura 3.2. (a) Intensidad de patrones $I(2 \theta)$ de rayos $X$ y (b) Patrones de entropía $E(2 \theta)$ de la señal de intensidad $I(2 \theta)$ para alúmina calcinada a $200^{\circ} \mathrm{C}$.

Los resultados anteriores muestran la habilidad del análisis de entropía aproximada multiescala para detectar regiones angulares en señales de intensidad con ruido intenso, que pueden ser atribuidas a las fases del material. El caso de la temperatura de calcinación a $500^{\circ} \mathrm{C}$ es más desafiante ya que los patrones de $X R D$ en la Figura 3.3. a muestran un sólo pico fuerte cerca de los 40 grados. La presencia de $\gamma^{*}$-bohemita cerca de los 10 grados se observa ligeramente como un pico pequeño en tal región angular. La Figura 3.3. b muestra el comportamiento de los patrones de entropía $E(2 \theta)$ obtenido por el análisis de entropía aproximada multiescala del patron de XRD de la Figura 3.3. a. Donde puede observarse 
3. Señales de intensidad de difracción de rayos $X$

regiones de mínimos, interesantemente estas regiones pueden estar asociadas a la presencia $\gamma$-alúmina en el material calcinado. El valor mínimo de entropía está localizado en la región de los 40 grados que corresponde a un fuerte y único pico, mostrado en la señal de intensidad de $X R D$. También se observa la presencia de $\gamma^{*}$-bohemita en el patrón de entropía $E(2 \theta)$ cerca de los 10 grados. Interesantemente, la entropía de la $\gamma^{*}$-bohemita aumentó de 0.44 a 0.75 en comparación con la temperatura de calcinación a $200^{\circ} \mathrm{C}$. Esto sugiere, como se esperaba, que fases de bohemita son transformadas a $\gamma$-alúmina conforme la temperatura de calcinación se incrementa dentro del rango de $100-500^{\circ} \mathrm{C}$.
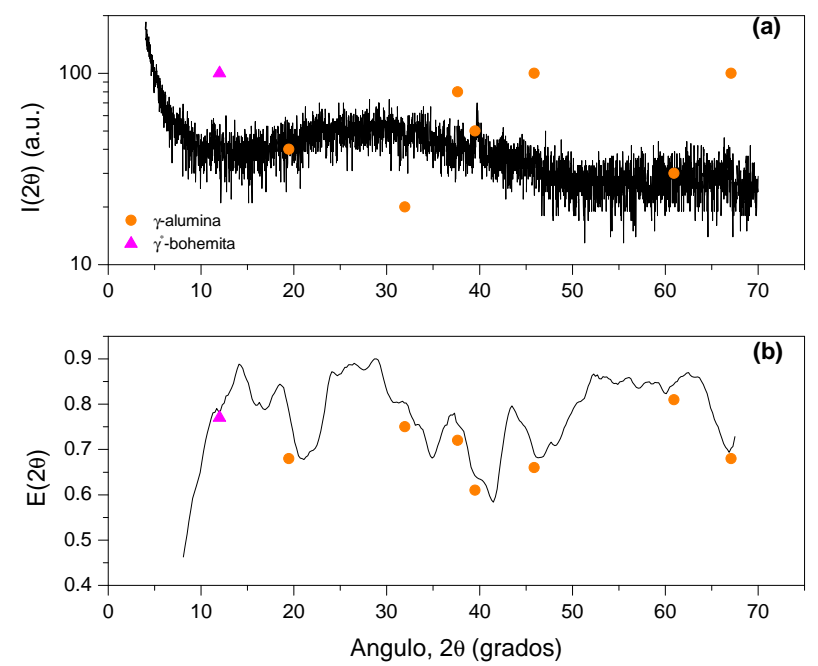

Figura 3.3. (a) Intensidad de patrones $I(2 \theta)$ de rayos $X$ y (b) Patrones de entropía $E(2 \theta)$ de la señal de intensidad $I(2 \theta)$ para alúmina calcinada a $500^{\circ} \mathrm{C}$.

Conforme la temperatura de calcinación se cambio, nuevas fases fueron obtenidas por la transformación a otras fases. De esta forma, la entropía de ciertas fases fue reducida como efecto de la transformación en la estructura de la materia. La Figura 3.4 presenta la evolución de la entropía para regiones en la cercanía de cuatro diferentes ángulos. Lo siguiente puede ser comentado:

3.4.a La entropía para la región cerca de 12 grados aumenta después de $200^{\circ} \mathrm{C}$, indicando una gradual destrucción de la $\gamma^{*}$-bohemita para producir $\gamma$-alúmina conforme la 

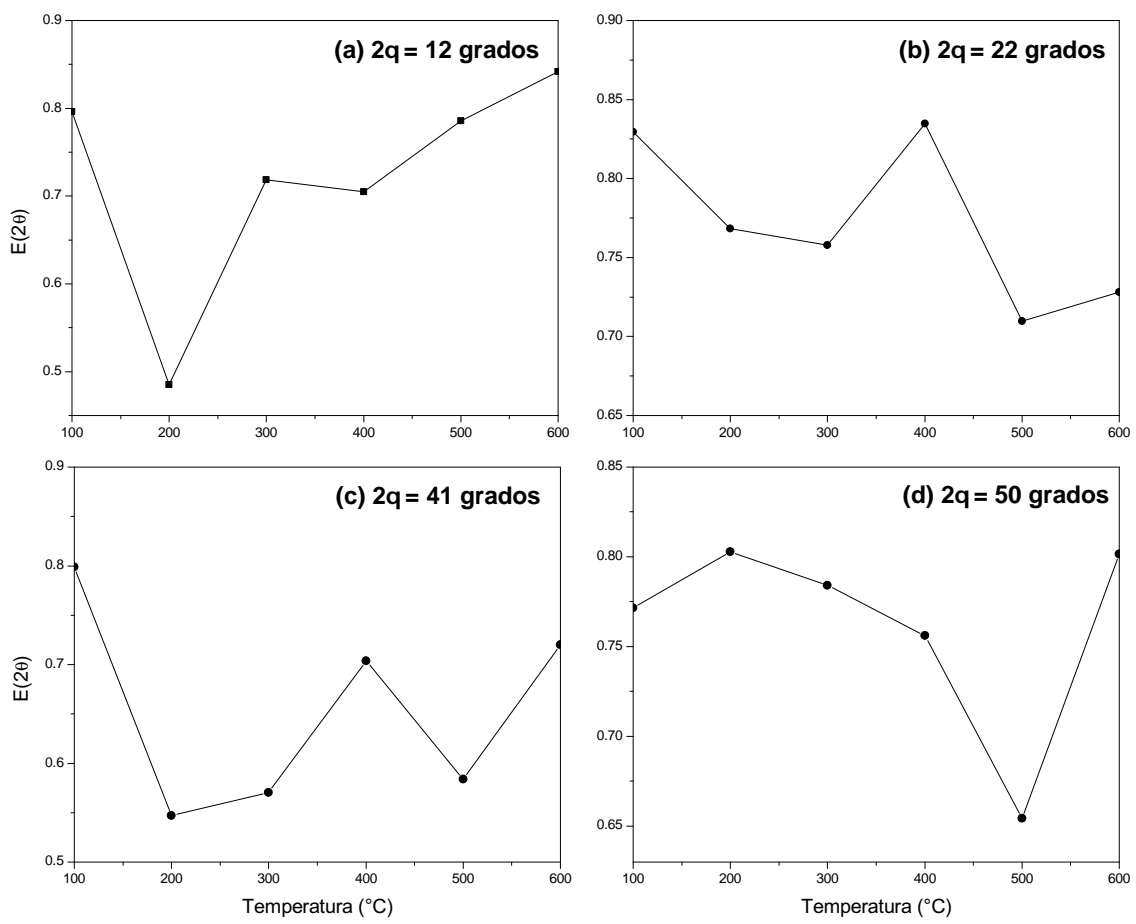

Figura 3.4. Patrones de entropía $E(2 \theta)$ en función de la temperatura de calcinación para cuatro diferentes regiones angulares.

temperatura se incrementa. Es evidente que $\gamma^{*}$-bohemita es el principal precursor para la formación de $\gamma$-alúmina ya que estas fases tienen una estructura cristalina semejante.

3.4.b La entropía de los grupos óxidos, localizado cerca de 22 grados, muestra un comportamiento no monótono, el cual podría reflejar transiciones complejas de estas fases a la fase $\gamma$-alúmina.

3.4.c La entropía cerca de los 40 grados muestra un comportamiento no monótono, el cual refleja la transición gradual de bayerita y $\gamma$-bayerita a la fase $\gamma$-alúmina. Observe que la entropía sufre un fuerte aumento cuando la temperatura de calcinación está por arriba de $500^{\circ} \mathrm{C}$, lo cual sugiere una extensa destrucción de la geometría cristalina. 
3. Señales de intensidad de difracción de rayos $X$

Esta observación apoya la práctica de usar temperaturas de calcinación no mayores a $500^{\circ} \mathrm{C}$ para preparar catalizadores con base alúmina (Gates y col., 1979).

3.4. d La entropía para la región cerca de los 50 grados muestra un incremento hasta un máximo en la temperatura de $200^{\circ} \mathrm{C}$ y posteriormente una disminución hasta un mínimo para la temperatura de $500^{\circ} \mathrm{C}$ y después un fuerte incremento en su comportamiento, indicando la gradual destrucción de $\gamma$-bohemita a estructuras de fases $\gamma$ conforme la temperatura de calcinación se incrementa hasta los $500^{\circ} \mathrm{C}$.

Los resultados anteriores muestran que el análisis de entropía aproximada multiescala puede ser usado como una herramienta para monitorear los cambios de fases de diferentes materiales durante el proceso de calcinación.

\subsection{Conclusiones}

Este Capítulo3 se presentó una metodología basada en el análisis de entropía aproximada multiescala para caracterizar los patrones de difracción de rayos $X$ con ruido intenso. La idea es detectar regiones angulares donde las fluctuaciones de intensidad de difracción de rayos $X$ no son aleatorias del todo, sino que exhiben cierto grado de regularidad. Para lograrlo, regularidades en las señales de intensidad se cuantificaron mediante un análisis de entropía aproximada multiescala que pueden ser asociados con patrones geométricos contenidos en la compleja estructura del material. El enfoque del análisis fue ilustrado con alúmina calcinada a diferentes temperaturas, mostrando que el método puede proveer información sobre la localización de fases de alúmina corroborados contra tarjetas PDF. 


\section{Parte II}

\section{Monitoreo de columnas de destilación y sistemas de corrosión electroquímica}


CAPÍTULO 4

COLUMNAS DE DESTILACIÓN

En este Capítulo 4 se presenta el estudio de monitoreo realizado a columnas de destilación binarias a través de análisis de entropía aproximada. En la Sección 4.1 se presenta una introducción de conceptos relevantes, factores que afectan su desempeño dinámico y así como de sistemas de monitoreo en columnas de destilación. Además en la Sección 4.2 se describen las características, así como la metodología empleada en el monitoreo de columnas de destilación binarias a través de análisis de entropía aproximada. Y por último, en la Sección 4.3 se muestran los resultados obtenidos en el análisis de entropía de señales de tiempo de las columnas de destilación analizadas.

\subsection{Introducción}

La destilación es una técnica común para la separación de corrientes líquidas con dos o más componentes y es una de las operaciones unitarias más importantes en la industria química, petroquímica, alimenticia y farmacéutica (Luyben, 1996). Su objetivo principal es separar de una mezcla sus componentes aprovechando sus distintas volatilidades, o bien separar los materiales volátiles de los no volátiles. Su modelación dinámica se caracteriza por ser multivariable, no lineal, con fuertes interacciones entre variables y sujeta a perturbaciones. 
4. Columnas de destilación

Por consiguiente, presenta respuestas dinámicas que dependen de combinaciones en los valores de los flujos, lo que implica un sistema altamente interactivo. Además a lo anterior puede sumarse la existencia de ruido inherente a las mediciones, tiempos de retardos en la medición de variables o en las acciones de control del sistema.

Por tales motivos, una columna de destilación binaria representa un interesante problema en el diseño de control no lineal, como resultado de la dinámica compleja que envuelve el sistema, el equilibrio termodinámico que envuelve las interacciones entre corrientes externas e internas del sistema, la alta no linealidad y el carácter distributivo de una columna de destilación que son representados por los perfiles de composición y temperatura Skogestad, 1997; Gupta y col., 2009).

El comportamiento dinámico en columnas de destilación puede ser afectado por una variedad de factores que afectan su desempeño dinámico, algunos ejemplos son:

- Condiciones de alimentación.

- Condiciones de flujos de líquido y vapor.

- Condiciones de platos.

- Condiciones mecánicas, ambientales, etc...

\subsubsection{Factores que afectan la columna de destilación}

\section{Cambios en las condiciones de alimentación}

Durante el monitoreo de columnas de destilación se pueden presentar perturbaciones en la corriente de alimentación y por lo tanto afectar directamente la composición de salida de productos. En la Figura 4.1 (Treybal, 1998) puede observarse que la condición de alimentación está directamente relacionada con el número de etapas necesarias para una separación. Por tal motivo, perturbaciones o cambios realizados en las condiciones de alimentación, estado y/o composición, tendrán un efecto negativo en la operación adecuada del sistema. Esto es, se necesitaría realizar una modificación en el número de etapas 
requeridas o en la localización del punto de alimentación, adaptación de boquillas para alcanzar las purezas especificadas en los productos.

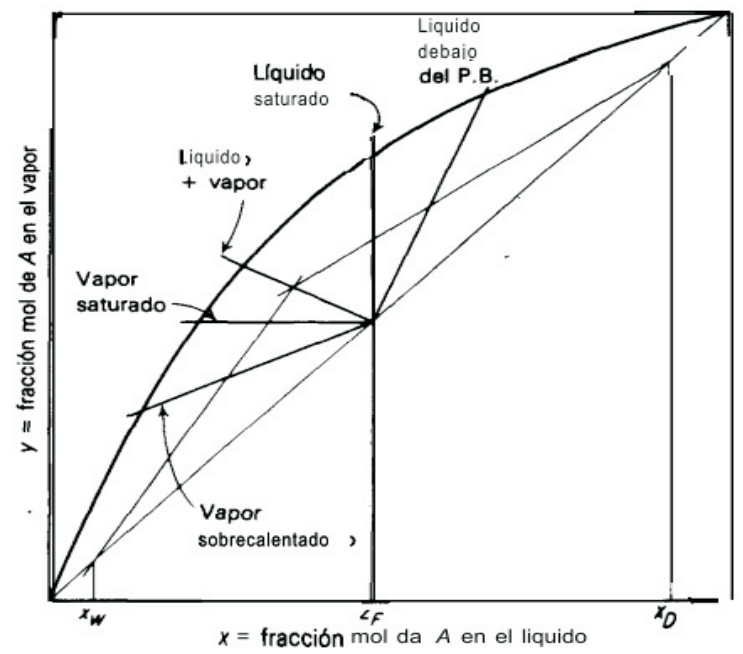

Figura 4.1. Localización de la línea $q$ para condiciones típicas de alimentación.

\section{Cambios en la relación de reflujo}

Cuando se realizan cambios en la relación de reflujo se pueden tener dos condiciones límites: reflujo mínimo o total. En la primera de ellas, cuando se tiene una relación mínima de reflujo se necesita un número infinito de etapas, $N=\infty$. Por lo tanto, la columna requerirá un número infinito de platos y en consecuencia el costo fijo es infinito, pero son mínimos los costos de operación, calor para el rehervidor, agua de enfriamiento, para el condensador, potencia para la bomba de reflujo. Mientras que, cuando se regresa como reflujo todo el producto principal a la columna y rehirviendo todo el producto residual (reflujo total) se requiere un mínimo de platos, $N_{m}$. Por consiguiente al aumentar la relación de reflujo, el número de platos decrece rápidamente, en cambio el diámetro de la columna, el condensador, la bomba de reflujo y el rehervidor deben de ser mayores. Por lo tanto, los costos fijos disminuyen hasta un mínimo y crecen nuevamente hasta infinito en el reflujo total (Treybal, 1998).

En la Figura 4.2 se muestra la relación directa entre los costos fijos, de funcionamiento y 
4. Columnas de destilación

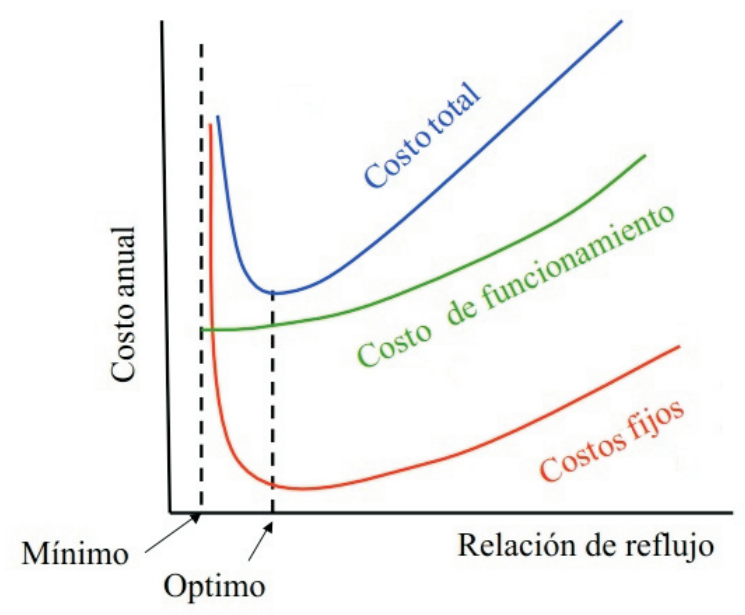

Figura 4.2. Relación de reflujo óptima

totales en función con la relación de reflujo. Por lo tanto, durante el diseño de una columna de destilación debe elegirse la relación de reflujo óptima o más económica, considerando un costo mínimo.

\section{Cambios en la relación de flujo de vapor}

En columnas de destilación el flujo entre el gas y el líquido se lleva a cabo a contracorriente, donde ocurre la difusión interfacial y los fluidos se separan. Sin embargo cuando se establecen valores incorrectos en los flujo de operación se presentan fenómenos como formación de espuma (foaming), arrastre (entrainment), lloriqueo (weeping/dumping) e inundación (flooding). Las relaciones entre los flujos de líquido y gas se muestran en forma esquemática en la Figura 4.3 (Treybal, 1998).

Pueden resumirse estos fenómenos como sigue. La profundidad elevada del líquido en los platos proporciona eficiencias de platos elevadas mediante tiempos largos de contacto, pero también causa una alta caída de presión por plato causando inundación. O bien, las velocidades elevadas del gas, dentro de límites razonables, proporcionan buen contacto vaporlíquido mediante dispersión excelente, pero ocasionan excesiva entrada del líquido al gas y una alta caída de presión; lo cual puede acarrear la condición de arrastre por espuma, que también es una situación inoperante (Treybal, 1998). 
4. Columnas de destilación

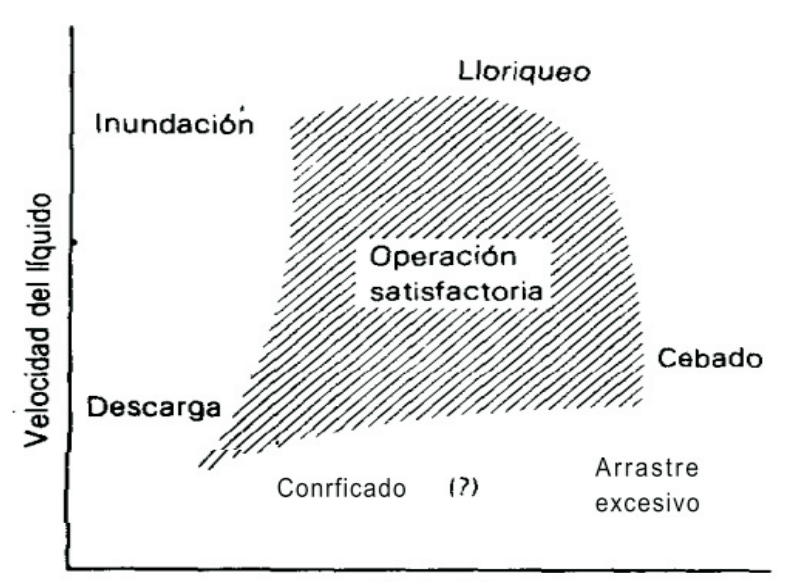

Flujo del gas

Figura 4.3. Características de operación en platos perforados.

Pueden ocurrir otras condiciones indeseables. A muy baja rapidez del líquido, el gas ascendente a través de los orificios del plato puede empujar al líquido hacia afuera y es deficiente el contacto entre el gas y el líquido. Si el flujo del gas es muy bajo, parte del líquido caerá a través de los orificios del plato (lloriqueo) (Treybal, 1998). Todos los tipos de platos están sujetos a alguna de estas dificultades. Por lo tanto, se deberá seleccionar los diferentes arreglos, dimensiones o condiciones de operación que de acuerdo a la experiencia establecen un término adecuado de funcionamiento.

\section{Otros factores}

Durante la operación de columnas de destilación se pueden presentar dificultades mecánicas, corrosión, ensuciamiento, desgaste del equipo, entre otras, que afectan la eficiencia de los platos y en consecuencia su rendimiento. Lo que contribuye a que no se alcancen las especificaciones de los productos con las purezas deseadas. Otro factor que afecta el rendimiento de la columna son los cambios en las condiciones climáticas pueden afectar directamente a los rehervidores. Esto es, deben de ser capaces de generar o regular una cantidad suficiente vapor durante estaciones con temperaturas muy altas o muy bajas. 
4. Columnas de destilación

\subsubsection{Variables a monitorear en columnas de destilación}

El diseño, monitoreo y control de una columna de destilación es de gran importancia, ya que permite mantener corrientes de productos con las purezas requeridas dentro de regulaciones ambientales, restricciones de economía y de operación. El objetivo de un sistema de monitoreo es detectar, identificar y corregir variaciones o fallas en el proceso en tiempo real que puedan afectar el rendimiento del sistema.

En el caso de la columna de destilación se miden, recopilan y analizan variables como: flujo de agua al condensador, presión de columna, concentración por etapa, temperatura de alimentación, entre otras variables. Lo cual implica una serie de datos de variables que se deben considerar para su monitoreo. También se debe considerar que los componentes de un sistema de destilación (tanques de depósito, válvulas, tuberías, rehervidores, condensadores, entre otros) frecuentemente se encuentran en contacto con el medio ambiente, haciendo susceptible al sistema a oscilaciones o fluctuaciones en las variables a controlar. Además se suma el ruido en las mediciones durante la operación del sistema, ya sea de origen eléctrico, térmico, por tiempos muertos o transmisión de señales. Lo cual puede implicar el manejo de señales dinámicas no lineales y no estacionarias, que además presentan ruido.

\subsection{Metodología}

En una columna de destilación se lleva a cabo un proceso de separación por etapas, lo que implica que se tengan balances de masa total, de componente y energía en cada etapa o plato (Doherty y Perkins, 1982). Sin embargo, existen muchos sistemas para los cuales es suficiente modelar la dinámica de masa total y de componente en cada plato, es decir, no es necesario incluir la dinámica de los balances de energía (Skogestad y Morari, 1988), bajo la llamada hipótesis de flujo equimolar (Halvorsen y Skogestad, 2000). Las columnas a analizar se modelaron bajo la suposición anteriormente descrita, y en el modelo matemático considerado solo se tomo en cuenta el balance de masa global y por componente, el balance hidráulico y el equilibrio termodinámico. 
4. Columnas de destilación

Se consideraron columnas como las que se muestran en la Figura 4.4, cuya composición $X_{F}$ es alimentada a una tasa $F$ en el plato $N_{F}$. Donde se producen dos efluentes fondo $(B)$ y destilado $(D)$ con composiciones $X_{B}$ y $X_{D}$, respectivamente. En cada caso se cuenta con NT platos incluyendo el rehervidor, un condensador total y una etapa de alimentación.

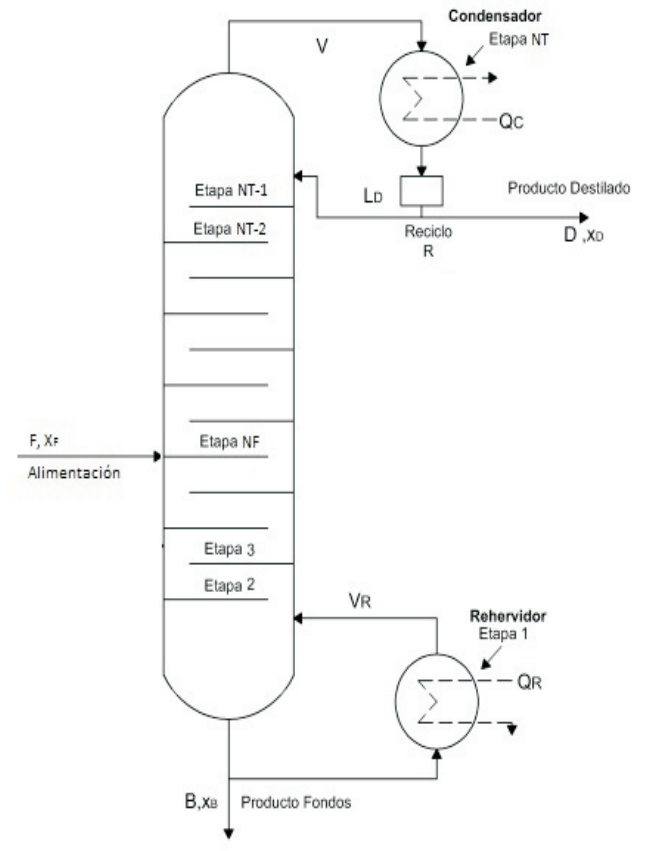

Figura 4.4. Diagrama esquemático de la columna de destilación binaria por etapas.

Como casos de estudio se consideran la separación de las siguientes mezclas: volatilidad relativa $\alpha=1.5$ (Columna A), benceno-tolueno (Columna B y C) y metanol-agua (Columna D). Las especificaciones de las columnas de destilación en estado estacionario se describen en el Apéndice A.1, donde se consideran flujos equimolares, presión constante, equilibrio termodinámico y mezclado perfecto en todos los platos, control de nivel perfecto en el rehervidor y el tanque de condensados, condensador total y masa despreciable de vapor en cada plato (Doherty y Perkins, 1982; Halvorsen y Skogestad, 2000).

Las señales de temperatura de las columnas fueron analizadas utilizando algoritmos de entropía implementado un esquema de ventana deslizante para estimar el contenido de entropía en la serie de tiempo (Pincus, 1991). Se seleccionaron este tipo de señales 
4. Columnas de destilación

para el análisis porque se consideró que, durante la operación este tipo de señales pueden ser recopiladas fácilmente en tiempo real y en forma continua a través de sensores de temperatura.

La metodología emplea el análisis de entropía para identificar perturbaciones o cambios asociados a inestabilidades en la operación de la columna. La idea principal es que patrones regulares contenidos en la serie temporal reflejan un cierto grado de orden en la dinámica de la señal. De esta forma, una señal temporal del sistema que se encuentra operando establemente corresponde a patrones con mayor regularidad asociados a una menor complejidad. Por otra parte, perturbaciones o inestabilidades en la señal corresponden a comportamientos aleatorios en la señal temporal de temperatura.

Basado en lo anterior, la metodología propuesta para realizar el monitoreo de columnas de destilación consiste en analizar series temporales de temperatura de los casos analizados (Apéndice A.1) mediante análisis de entropía aproximada. De esta forma, bajos valores de entropía serán asociados con procesos estables de operación. Mientras que, altos valores de entropía serán relacionados con fallas en sensores, inestabilidades del sistema o perturbaciones, o cualquier condición que pudiera afectar el rendimiento del sistema.

\subsection{Resultados}

\subsubsection{Análisis de entropía aproximada}

El análisis de entropía aproximada $(A p E n)$ se emplea en señales temporales con el objetivo de identificar inestabilidades o irregularidades como consecuencia de perturbaciones, cambios de operación o fallas de operación que se producen durante la operación en columnas de destilación. Como se mencionó anteriormente en la Sección 4.2, el método se basa en examinar las variaciones de entropía con respecto a una escala de tiempo y una ventana deslizante de tamaño $N_{w}$. De esta manera, como requisito previo para realizar cálculos de entropía, se debe seleccionar un tamaño adecuado de ventana deslizante $N_{w}$.

Para evaluar los efectos del tamaño de ventana, la entropía se calculó a través de todos 
los tamaños de ventana $N_{w}$ contenidos en toda la serie temporal, después se empleó un filtro pasa-bajas para una escala de tiempo $\tau=n$. Posteriormente se promedió sobre todas las posibles ventanas contenidas en una serie de tiempo $Y_{f}$. La Figura 4.5 presenta el comportamiento de la entropía aproximada $\operatorname{ApEn}(\tau)$ de la señal de temperatura de la columna C como una función del tamaño de ventana $N_{w}$ para cinco diferentes escalas de tiempo $(\tau)$ de 1, 3, 6, 9 y 12 horas que corresponden a 1000, 1510, 2500, 3520 y 4000 datos respectivamente.

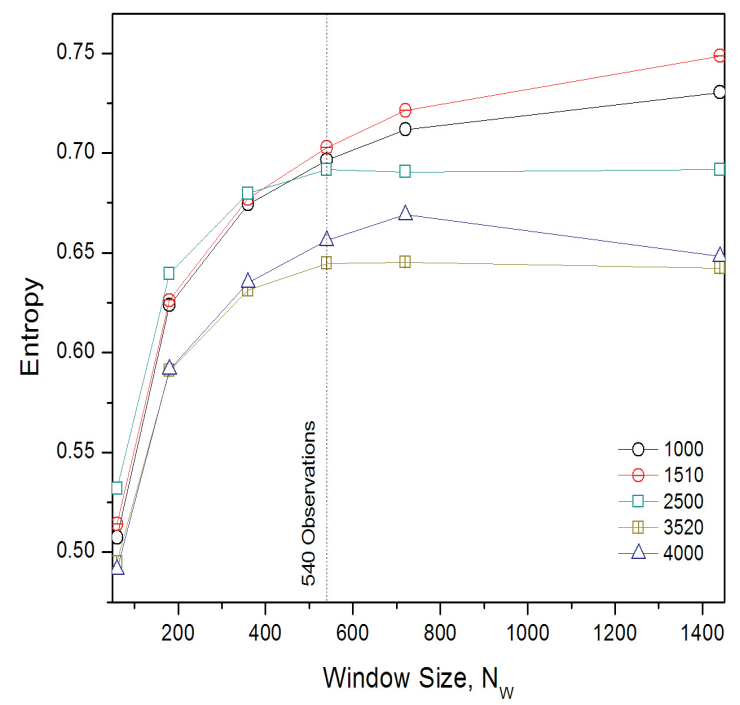

Figura 4.5. Entropía aproximada $A p E n(\tau)$ como una función de la longitud del tamaño de ventana $N_{w}$, para cuatro diferentes escalas de tiempo $(\tau)$ para la señal de temperatura de la columna C.

En las escalas de tiempo seleccionadas, la entropía presenta un comportamiento bien definido de tal manera que aumenta con respecto a la escala de tiempo $(\tau)$ a partir de aproximadamente $N w>540$. Esto es, la entropía es una función creciente de la ventana de tamaño $N_{w}$, donde los patrones existentes no son destruidos a medida que aumenta $N_{w}$. Por ejemplo, para $N_{w}>540$, la entropía que corresponde a 1 hora es menor que la entropía de 3 horas. Se seleccionó un tamaño de ventana de $N_{w}=540$ datos $(\approx 1 / 2 \mathrm{hr})$ para los cálculos de entropía de la columna A, lo que garantiza que la entropía media está disminuyendo con respecto a $\tau$. Un valor más alto de $N_{w}$ puede ser elegido, aunque puede reducir la localidad 
4. Columnas de destilación

de las fluctuaciones de la entropía con respecto al tiempo. En la Figura 4.5 se observa que los cálculos de entropía se estabilizan a partir de aproximadamente 540 datos. Resultados similares fueron encontrados en los otros casos de estudio (Apéndice A.2).

Una vez seleccionado el tamaño de ventana para los cálculos de entropía, se investigaron los siguientes aspectos en las columnas de destilación como son complejidad de señales, irregularidades de señales, así como cambios realizados en: condiciones de alimentación, relación de reflujo y flujo de vapor. El sistema de monitoreo debe de ser capaz de detectar tales situaciones durante la operación de las columnas de destilación.

\subsubsection{Complejidad de señales}

La composición de alimentación $\left(X_{F}\right)$ se sometió al efecto de ruido (secuencias de ruido blanco con desviación estándar de 0.0005, inyectadas cada 0.50 min) para simular el ruido inherente a la operación del sistema. Esto es, la perturbación realizada puede asociarse al ruido presente en procesos reales durante la operación o medición de sus variables. La Figura 4.6 muestra una señal temporal de temperatura de la columna de destilación, la cual es aparentemente aleatoria sin ninguna correlación entre sí. Para lo cual, el estadístico de entropía ha demostrado que es capaz de manejar datos en presencia de ruido, con tendencia y que es capaz de proveer información necesaria para caracterizar al sistema (Goldberger y col., 2002; Richman y Moorman, 2000; Pincus y Kalman, 2004; Yan y Gao, 2007).

Para el análisis de complejidad en señales por medio de análisis de entropía aproximada se parte de la siguiente idea. Se considera que la columna de destilación, cuyo objetivo es separar una mezcla de sustancias en sus componentes, actuará como un sistema ordenador de estados, reduciendo la gama de probabilidades de patrones de moléculas. Donde la sección del domo y fondo procuraran una mezcla con mayor cantidad del componente mas volátil y pesado, respectivamente. De tal forma que, el destilado y el producto de fondos son sustancias refinadas que contienen mas información ya que se reduce la gama de probabilidades y además tienen una menor entropía de mezcla al restringirse la gama de microestados posibles.

En la Figura 4.7 se muestran los resultados del análisis de $A p E n$ de las señales de 


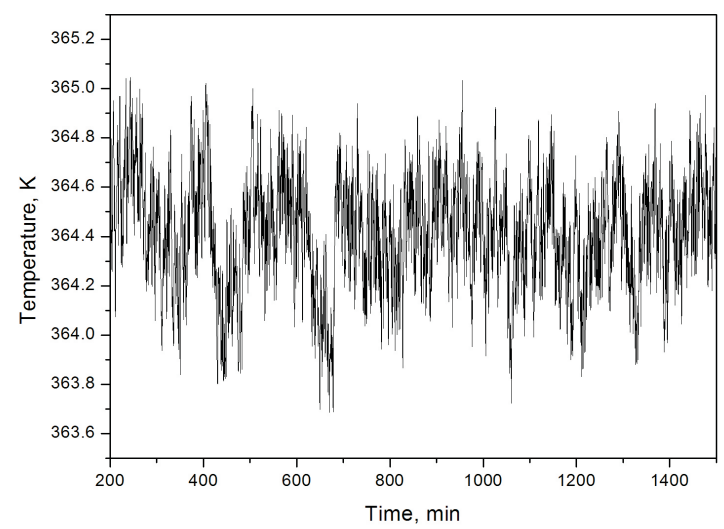

Figura 4.6. Señal de temperatura en columna de destilación

temperaturas en cada plato de la columna de destilación C. Puede observarse que para la sección de rectificación y agotamiento (Figuras 4.7a y 4.7b, respectivamente) la señal de mayor entropía (mayor complejidad) corresponde al plato de alimentación. Lo anterior se debe a que en esa etapa se encuentra una mezcla aleatoria de microestados con diferentes patrones o configuraciones de los componentes a separar. Por lo cual, es lógico encontrar que los mayores valores de entropía (mayor complejidad) se encuentran en la etapa de alimentación.

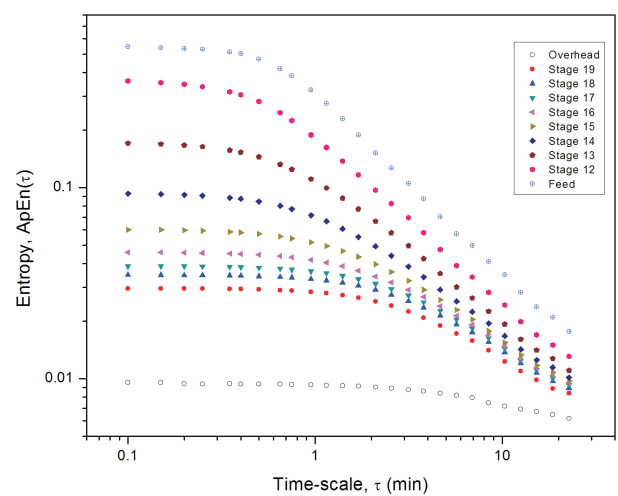

(a) Rectifying section

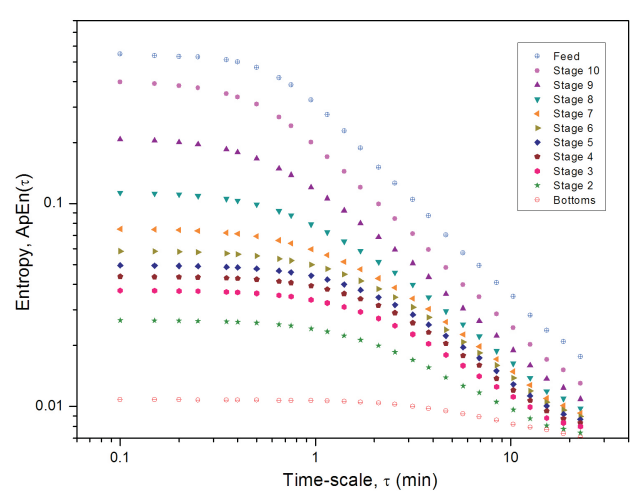

(b) Stripping section

Figura 4.7. Entropía a diferentes escalas $(\tau)$ de señales de temperatura para las secciones de rectificación y agotamiento de la columna C. 
Mientras que en las secciones de enriquecimiento y agotamiento se presentan los menores valores de entropía asociados con menores irregularidades o fluctuaciones en las series temporales, lo cual refleja una tendencia hacia la uniformidad de patrones. Esto es, en el caso de las señales de temperatura del destilado y fondo indican una uniformidad de patrones (menor entropía) asociados con el componente más volátil y menos volátil, respectivamente.

Por otra parte, se realizaron análisis de entropía aproximada multiescala a las señales de temperatura de la columna C. Los resultados se muestran en la Figura 4.8, donde puede observarse claramente que la señal de alimentación presenta el mayor valor de entropía en la microescala, a escalas de tiempo menores $\left(\tau<10^{0}\right)$. Es decir, la alimentación corresponde a la ausencia de patrones estructurados y por tanto mayor entropía.

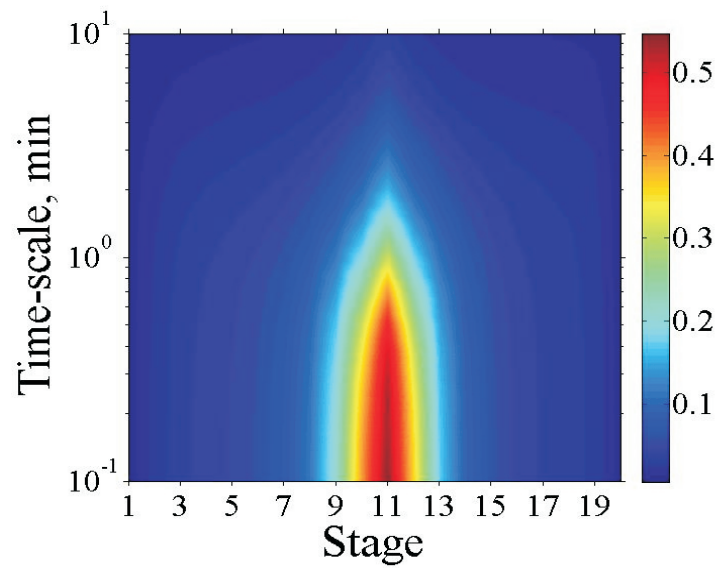

Figura 4.8. Entropía aproximada multiescala de la columna de destilación C.

Mientras que las señales del domo y fondo de la columna presentan menores valores de entropía, las cuales corresponden a señales de mezclas ricas en el componente más volátil y pesado, respectivamente y que por lo tanto corresponderán a la presencia de patrones estructurados (menor entropía).

Los resultados obtenidos con el análisis de MAE (Figura 4.8) concuerdan con los obtenidos con el análisis de ApEn (Figura 4.7). Lo anterior demuestra que, de acuerdo a la localización de las señales de temperatura en la columna, su distribución de complejidad 
4. Columnas de destilación

está asociada a su localización en la misma. El análisis de entropía para evaluar la complejidad de señales en la columna se extendió a los otros casos de estudio (Columnas A, B y D), encontrándose resultados similares a los de la columna C (Apéndice A.3).

Estos resultados demuestran que series temporales aparentemente aleatorias son señales dinámicas de flujo de materia-energía que siempre transporta información. De forma tal que, existe una relación entre la estructuración material (termodinámica) y la estructuración conceptual (informativa) del sistema.

\subsubsection{Perturbaciones en las condiciones de alimentación}

En esta sección se utilizó el análisis de entropía aproximada para identificar perturbaciones o factores, presentados en la Sección 4.1.1, que pueden afectar el control de la composición en las columnas de destilación, cuyo objetivo es mantener las purezas especificadas de los productos.

Usualmente en el sistema binario, las variables manipuladas son la relación de reflujo y el flujo de vapor, las variables controladas son las composiciones en el domo y fondo de la columna y la entrada de alimentación se mantiene constante, y sus variaciones alrededor del valor nominal son consideradas como perturbaciones.

Se emplearon series de tiempo de temperaturas de las etapas de las columnas, las cuales son fáciles de medir a través de sensores. El objetivo del análisis de entropía con fines de monitoreo es detectar situaciones que afecten el rendimiento de la columna, en este caso debido a perturbaciones o variaciones en el flujo y la composición de alimentación.

Para mostrar el desempeño del análisis de $A p E n$ se realizaron algunas simulaciones numéricas para la columna de destilación binaria C. Las condiciones en estado estacionario se describen en el Apéndice A.1. En la Figura 4.9 se muestran las señales de la composición de destilado y la entropía cuando la corriente de alimentación se sometió al ruido blanco y al siguiente conjunto de perturbaciones en escalón:

Figura 4.9a en t=100 min, se disminuyó la composición de alimentación $X_{F}$ de acuerdo a un $20 \%$ con respecto a su valor nominal; en t=300 min, la composición de alimentación 
4. Columnas de destilación

$X_{F}$ regresó a su valor nominal.

Figura $4.9 \mathrm{~b}$ cada $100 \mathrm{~min}$, se disminuyó la composición de alimentación a $X_{F}=0.35$ y despúes de 15 min la composición de alimentación $X_{F}$ regresó a su valor nominal; cada 200 min, se aumentó la composición de alimentación a $X_{F}=0.65$ y despúes de 15 min la composición de alimentación $X_{F}$ regresó a su valor nominal.

En la Figura 4.9a se muestran los efectos de las perturbaciones, así como el análisis de entropía de la serie de tiempo de temperatura del destilado cuando se realizaron cambios en la composición de alimentación de forma persistente por un largo periodo de tiempo. Donde puede observarse que, el estadístico de entropía puede detectar cambios en las condiciones de alimentación de manera continua. Lo cual se ve reflejado con altos valores de entropía, asociados con irregularidades o fluctuaciones en la señal temporal de temperatura del destilado como consecuencia de perturbaciones en forma continua en la composición de la alimentación de la columna de destilación. Además en la Figura 4.9b se exhibe el comportamiento de la señal temporal de temperatura en el destilado cuando se llevan a cabo pequeñas perturbaciones en cortos periodos de tiempo ( $<15 \mathrm{~min})$. Los resultados de entropía muestran que los mayores cambios de entropía están asociados a los tiempos en que se efectúan las perturbaciones en la alimentación, y por lo tanto corresponden a irregularidades en la señal temporal de temperatura del destilado.

Por otra parte para examinar los efectos de los cambios en la composición de alimentación en la columna se llevo a cabo el análisis de entropía aproximada multiescala. Se analizaron las señales temporales de temperatura de toda la columna en dos condiciones de operación. La primera de ellas es cuando se disminuye la composición de alimentación a $X_{F}=0.40$ y la segunda es cuando se aumenta a $X_{F}=0.60$. En la Figura 4.10 puede observarse el perfil de entropía de toda la columna a diferentes escalas de tiempo. En ambos casos cuando se aumenta o disminuye la composición de alimentación se observa que la distribución de entropía se desbalancea, es decir la distribución no se centra alrededor de la etapa de alimentación como ocurre cuando la columna se encuentra operando correctamente (Figura 4.8). Por el 
4. Columnas de destilación
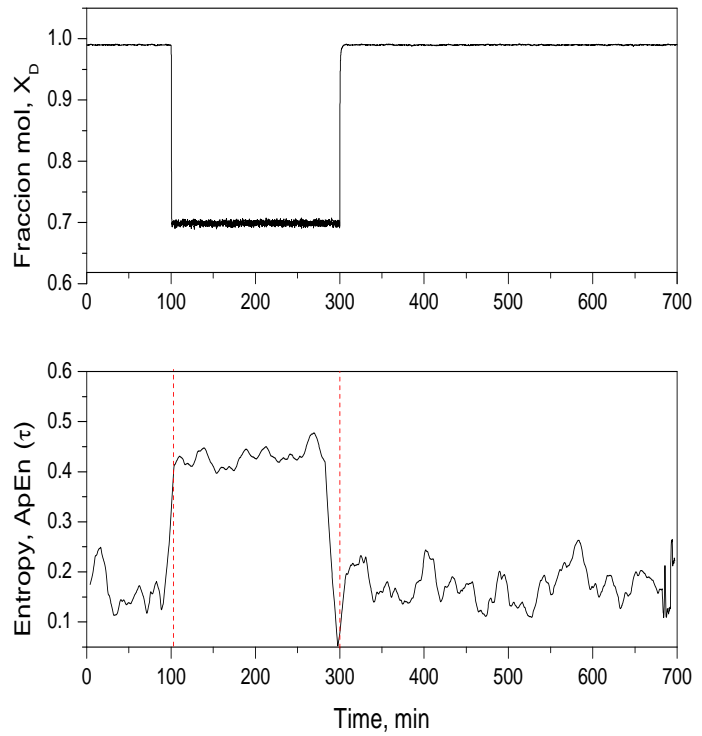

(a) Persistente
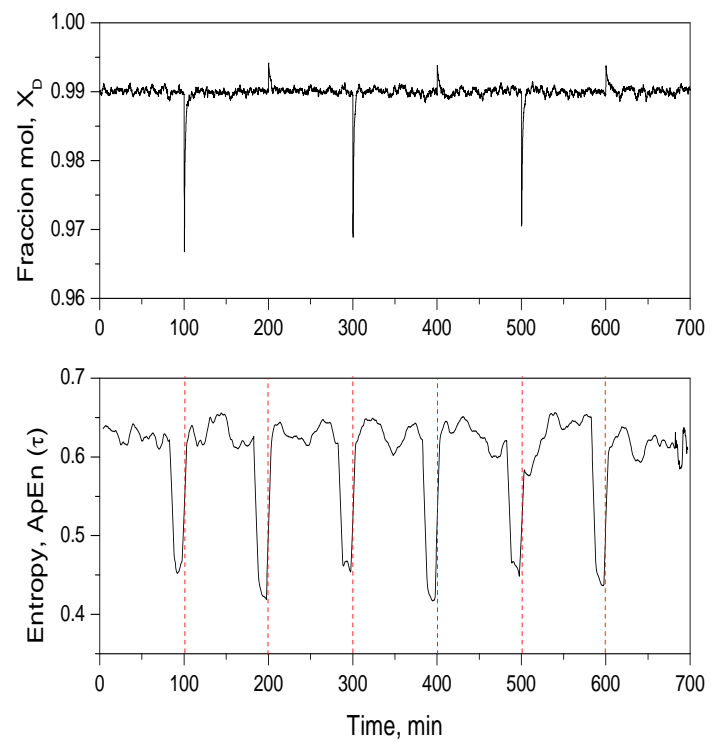

(b) Corta

Figura 4.9. ApEn cuando se realizan perturbaciones en la composición de alimentación de la Columna C.

contrario tiende a cargarse hacia un extremo, ya sea la sección de enriquecimiento o agotamiento, según sea el caso. 


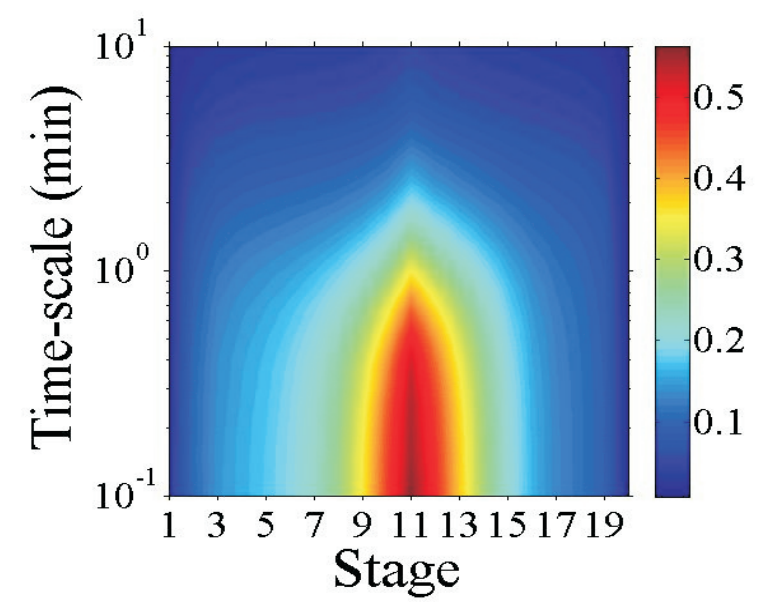

(a) $X_{F}=0.40$

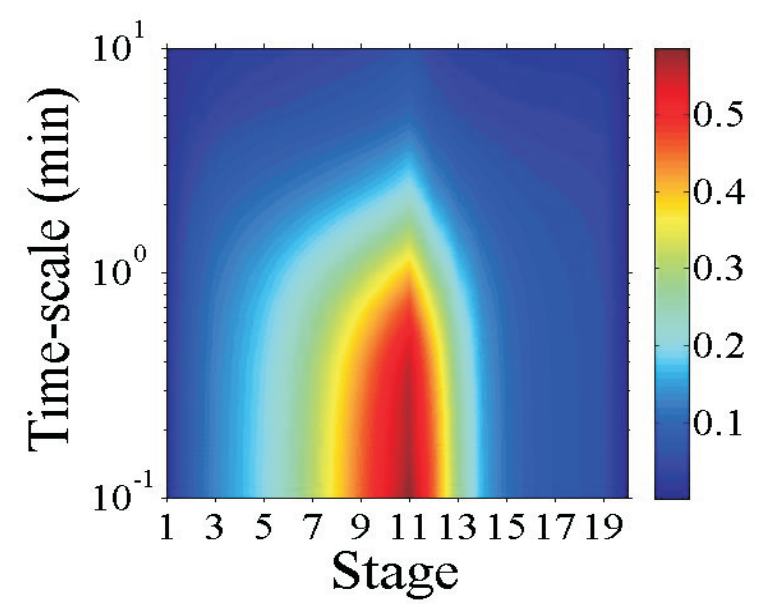

(b) $X_{F}=0.60$

Figura 4.10. Entropía aproximada multiescala de la columna de destilación $\mathrm{C}$ cuando se realizan cambios en la composición de alimentación.

Posteriormente se analizaron señales temporales en diferentes etapas de la columna con el objetivo de corroborar si los análisis de entropía detectaban de igual manera cambios en las condiciones de alimentación en cualquier etapa de la columna de destilación. Para lo cual se llevaron a cabo cambios en la composición de alimentación del $\pm 50 \%$ cada 500 min, además del ruido blanco impuesto.

La Figura 4.11 muestra los resultados del monitoreo de la columna mediante el análisis 
4. Columnas de destilación

de entropía en 4 diferentes etapas de la columna de destilación C. Puede observarse que cuando se llevaron a cabo las perturbaciones en las condiciones de alimentación estas se ven reflejadas como altos valores de entropía, lo cual indica que en ese punto la señal presenta fluctuaciones o irregularidades que pueden ser asociadas a perturbaciones en la corriente de alimentación.

Por último se llevaron a cabo los siguientes cambios en el flujo de alimentación de la columna:

- en t=100 min el flujo de alimentación se aumentó a $F=1.0 \mathrm{~mol} / \mathrm{min}$ y en t=300 min el flujo regresó a su valor nominal.

- en t=400 min el flujo de alimentación se disminuyó a $F=0.4 \mathrm{~mol} / \mathrm{min}$ y en t=600 min el flujo regresó a su valor nominal.

En la Figura 4.12 se muestra la señal temporal de temperatura del destilado y su correspondiente valor de entropía. Puede observarse que, cuando se lleva a cabo la perturbación en el flujo de alimentación, el estadístico de entropía es capaz de detectar dicha perturbación. Es decir, cuando se presenta la perturbación está se ve reflejada con altos valores de entropía lo que corresponde a irregularidades en la señal.

Por lo tanto, de acuerdo a los resultados obtenidos en esta sección se concluye que el estadístico de entropía es capaz de detectar fluctuaciones o irregularidades en las series de tiempo relacionados con cambios de operación en el flujo o composición de la corriente de alimentación.

\section{Cambios en la relación de reflujo y flujo de vapor}

En esta sección se estudia el monitoreo de la columna de destilación cuando se llevan a cabo cambios en las variables manipuladas como son la relación de reflujo y el flujo de vapor. 
4. Columnas de destilación

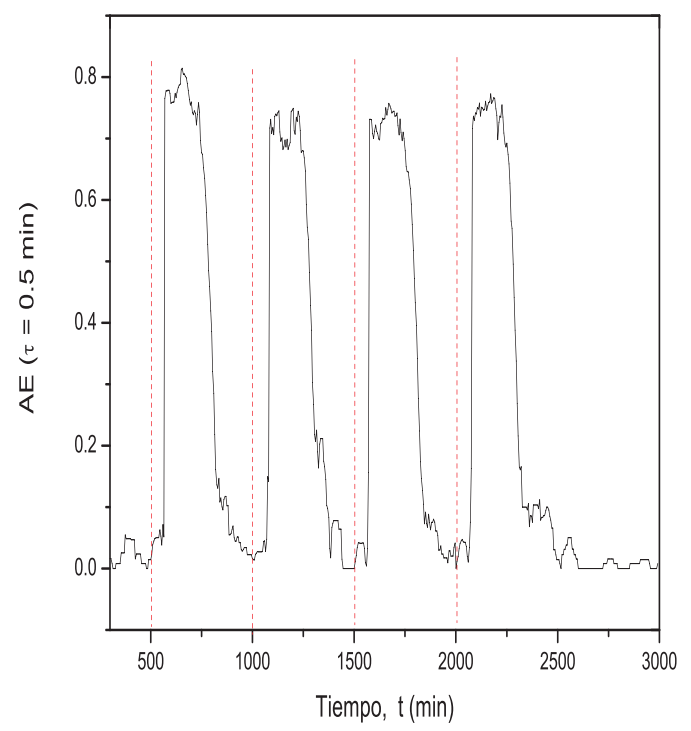

(a) NF-6

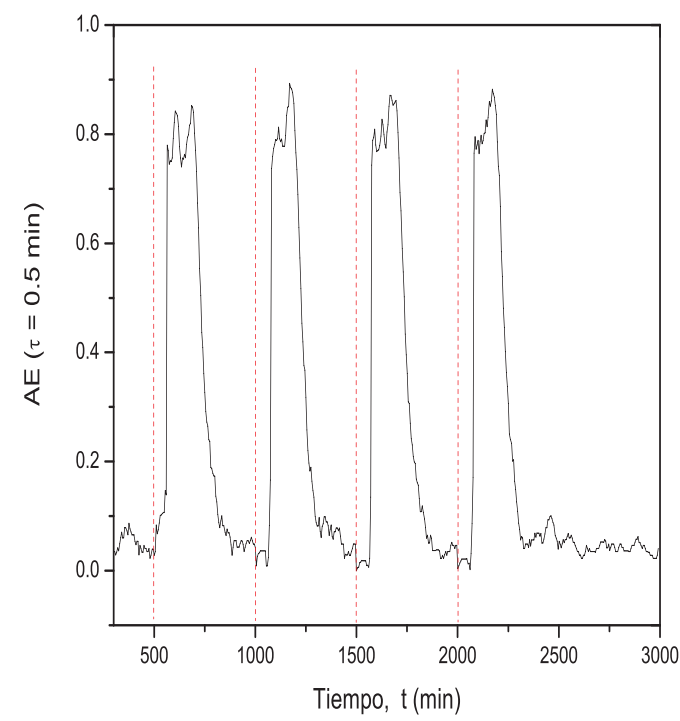

(c) $\mathrm{NF}+3$

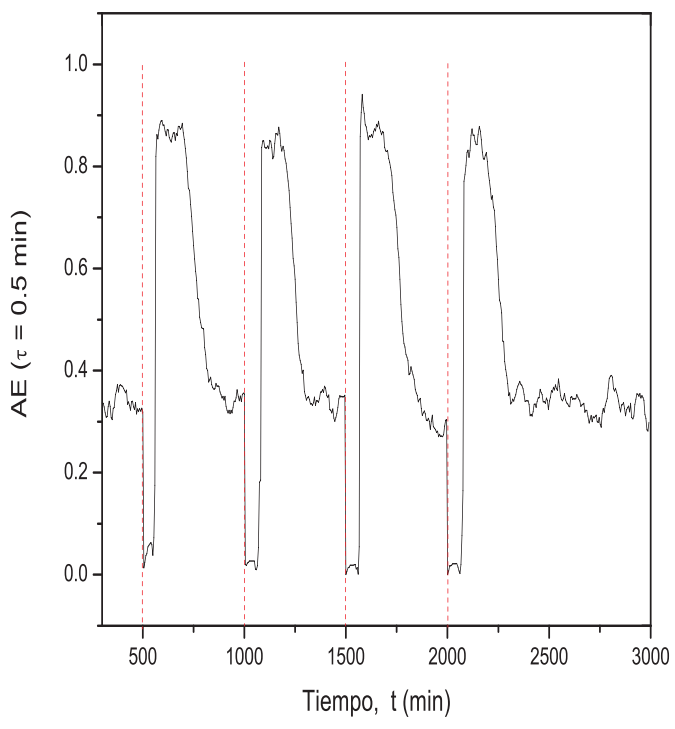

(b) NF-3

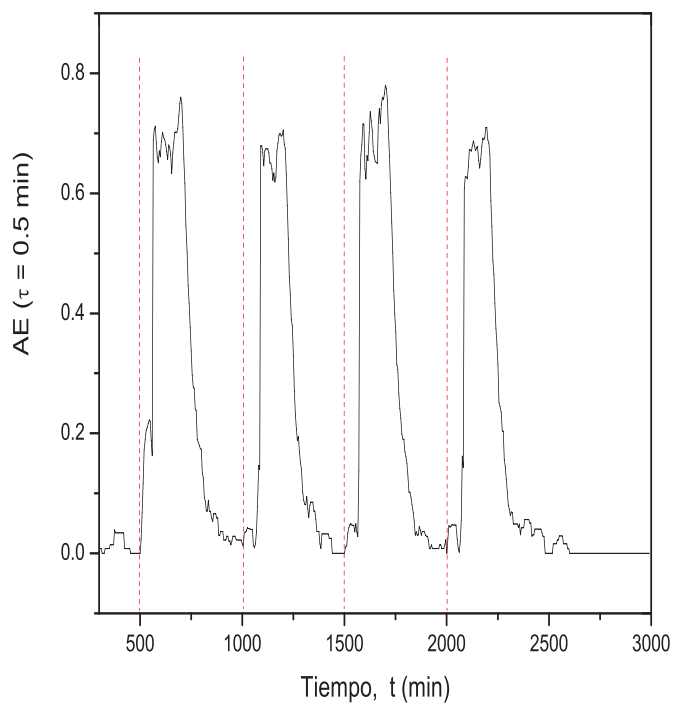

(d) $\mathrm{NF}+6$

Figura 4.11. ApEn en diferentes etapas de la columna cuando se llevan a cabo cambios en la composición de alimentación de la Columna C. 
4. Columnas de destilación
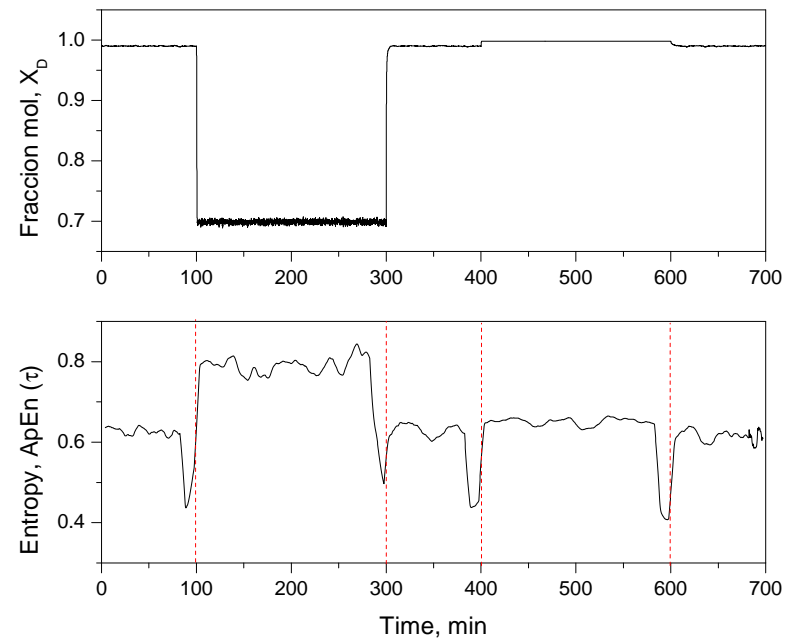

Figura 4.12. ApEn cuando se realizan cambios en el flujo de alimentación en la Columna C.

Se llevaron a cabo cambios en las variables manipuladas alrededor del $\pm 30 \%$ de sus valores nominales cada 500 min.

En la Figura 4.13 se muestran los resultados obtenidos con el análisis de entropía multiescala de algunas señales de la columna $\mathrm{C}$ cuando se llevan a cabo cambios en la variables manipuladas (relación de reflujo y flujo de vapor). Puede observarse que los mayores valores de entropía están asociados a los cambios en las variables y que por lo tanto no exhiben una regularidad de patrones.

\subsection{Conclusiones}

Los resultados obtenidos en este capítulo demuestran que el análisis de entropía aproximada puede ser utilizado como sistema de monitoreo en columnas de destilación. Debido a que dicho análisis puede detectar patrones contenidos en las series de tiempo de temperatura de columnas de destilación. La idea principal para interpretar los resultados de entropía son los siguientes: altos valores de entropía se asociaron a cambios o perturbaciones (irregularidades o fluctuaciones) en la señal monitoreada como consecuencia de cambios de operación en las variables manipuladas (ejem., relación de reflujo y flujo de vapor) 
4. Columnas de destilación

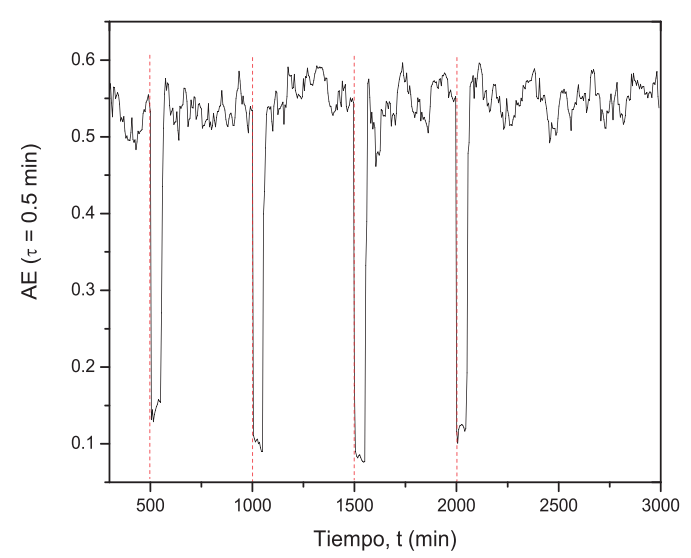

(a) NF-6

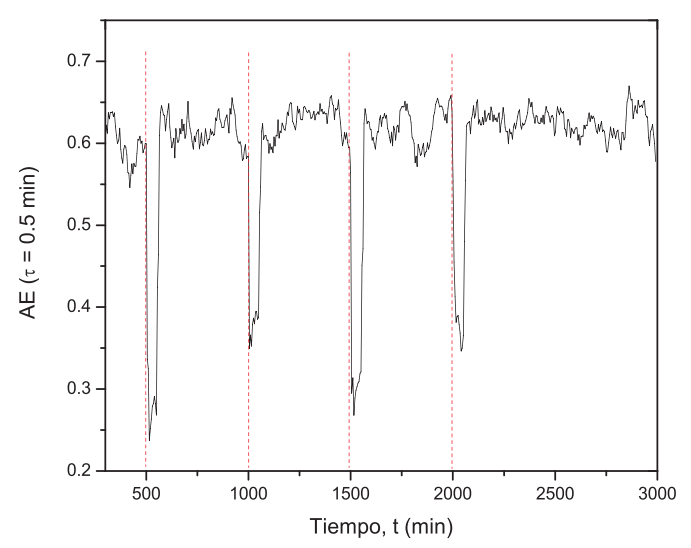

(c) $\mathrm{NF}+3$

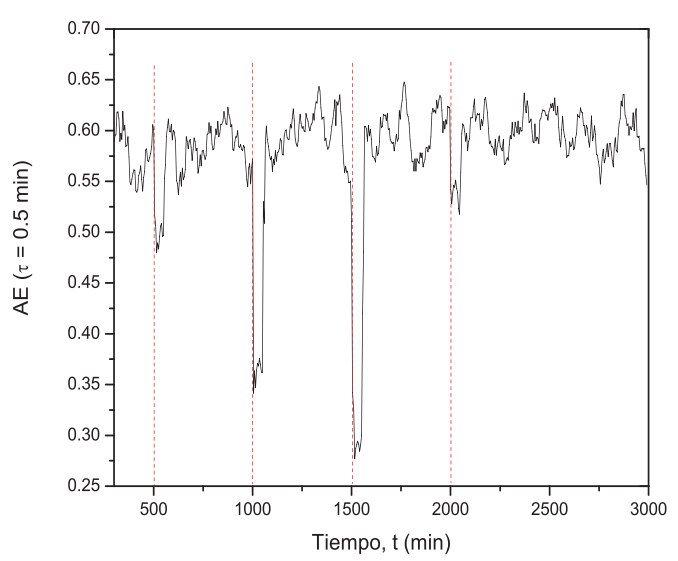

(b) NF-3

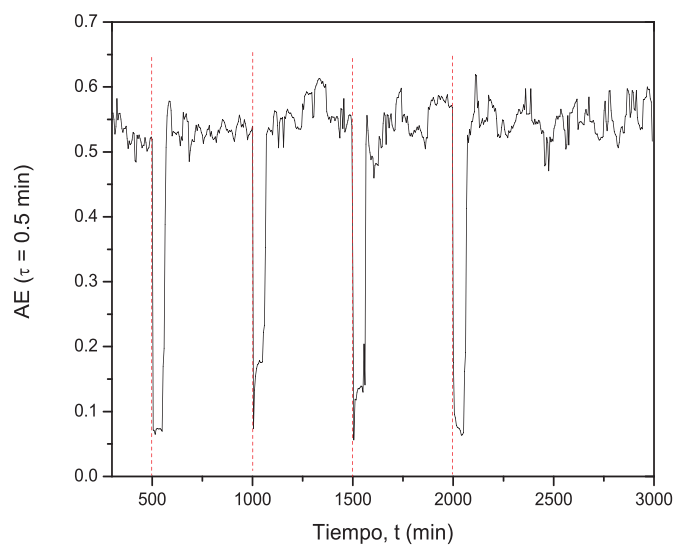

(d) $\mathrm{NF}+6$

Figura 4.13. ApEn cuando se llevan a cabo cambios de operación en la relación de reflujo y flujo de vapor de la Columna C.

o perturbaciones (ejem., alimentación). Mientras que bajos valores de entropía (patrones estructurados) correspondieron a situaciones normales de operación donde se presentan estructuras regulares, que corresponde a un sistema en operación normal en ausencia de irregularidades o cambios abruptos en la señal de proceso. 


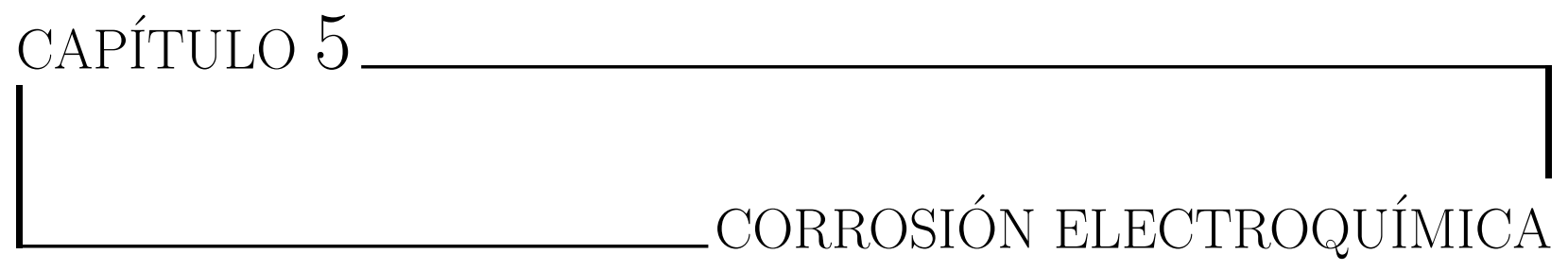

En este Capítulo 5 se aborda el análisis de las series de tiempo que se obtienen a partir de la técnica de Ruido Electroquímico ( $E N$, Electrochemical Noise) del acero inoxidable $A P I$ $X 52$, sumergido en solución de bicarbonato con y sin iones $C l^{-}$. En la Sección 5.1 se presenta una pequeña introducción de temas como son el monitoreo de la corrosión, tipos de corrosión, así como de algunas investigaciones importantes relacionadas con el tema. Posteriormente en la Sección 5.2 se explica la metodología empleada en el análisis de series de $E N$ de la señal cruda, así como de técnicas de entropía aproximada. Despúes en la Sección 5.3 se presentan los resultados del análisis de entropía aproximada y se comparan contra los obtenidos en el análisis de la señal cruda. Y finalmente en la Sección 5.4 se presentan las conclusiones obtenidas en este Capítulo.

\subsection{Introducción}

\subsubsection{Monitoreo de la corrosión}

Otro caso interesante de estudio es el monitoreo de corrosión en estructuras con protección catódica o anódica, aleaciones y en procesos con altos niveles de presión, temperatura, 
5. Corrosión electroquímica

toxicidad, inflamabilidad o altamente corrosivos. La medición de la corrosión permite obtener información acerca de que tan corrosivo es el ambiente del sistema y a que tasa o rapidez se experimenta la pérdida de metal. El monitoreo de la corrosión se mantiene como una herramienta indispensable en la industria papelera, petroquímica y química, producción de gas y petróleo, refinería y en servicios auxiliares.

La corrosión es la destrucción de un material que resulta de la exposición e interacción con el ambiente. Es un proceso electroquímico mediante el cual los metales reaccionan con el ambiente para formar óxidos u otros compuestos. Cuando el metal es expuesto a un electrólito, los átomos del metal en el sitio del ánodo pierden electrones, los cuales son absorbidos por otros átomos del metal en el sitio del cátodo.

Existe un gran número de técnicas para el monitoreo de la corrosión, las cuales pueden ser clasificadas como: intrusivas/no intrusivas y métodos directos e indirectos Kane, 2007; Eden y col., 1992). Las pruebas no destructivas como ultrasonido, radiografía, termografía son consideradas como técnicas de monitoreo indirectas, ya que son utilizadas para inferir el ambiente corrosivo que pueda existir. Los métodos intrusivos y directos se basan en la exposición con el ambiente corrosivo para obtener información acerca de la pérdida de metal y/o tasa de corrosión del sistema. Algunos ejemplos de tales métodos incluyen los cupones de corrosión (medición de pérdida de peso), resistencia eléctrica (probetas $(E / R)$, resistencia de polarización lineal (probetas LPR), análisis de ruido electroquímico (electrochemical noise, $E N)$, entre otras.

El EN es definido generalmente como variaciones estocásticas en señales de potencial y corriente de los sistemas de corrosión electroquímica. La corrosión es considerada como un proceso en equilibrio y de cinética constante; mientras que, en el caso del $E N$ se registran desequilibrios en las reacciones electroquímicas en intervalos de tiempos muy cortos. La interfase metal (capa de productos de corrosión) y electrólito es el punto donde se producen en forma continua las señales de $E N$ en forma de pequeñas variaciones (transientes) de potencial y corriente.

El $E N$ es una de las técnicas que ha demostrado proveer información valiosa acerca de los 
5. Corrosión electroquímica

tipos de corrosión, nucleación, propagación y repasivación de los procesos de picados. Esto es, permite obtener información en tiempo real sobre la morfología de la corrosión (corrosión localizada por picaduras o uniforme) en electrólitos, a través del análisis de la velocidad e intensidad de señales de EN, tipo de material, características de su superficie en contacto con el medio, así como diferentes factores de diseño (Goellner, 2004).

Existen varios tipos de técnicas para el análisis de $E N$ para extraer información útil como tipos de corrosión y velocidades de corrosión desde las señales electroquímicas. Entre las más comúnmente usadas se encuentran el dominio estadístico como la media, varianza, kurtosis, skewness y la densidad espectral de potencia en el dominio de la frecuencia.

Diversas investigaciones han empleado el $E N$ en el análisis de propiedades de diversos parámetros que han sido propuestos como indicadores de tipos de corrosión en metales (Cottis y col. 2001).

\subsubsection{Corrosión uniforme}

La corrosión uniforme es una forma de corrosión electroquímica que tiene lugar con igual intensidad en la totalidad de la superficie expuesta y que con frecuencia deja una escama o depósito residual. A nivel microscópico, las reacciones de oxidación y reducción tienen lugar de manera aleatoria sobre la superficie (Callister, 2007).

Dos etapas teóricas del mecanismo de corrosión uniforme son las siguientes (Jirarungsatian y Prateepasen, 2010):

a La etapa inicial en el que la superficie del metal es atacado por soluciones químicas. Los sitios anódicos y catódicos son virtualmente inseparables.

b Un núcleo de la corrosión se propaga en la superficie del metal como un motor de corrosión uniforme. Después de que este fenómeno se ha producido, la re-pasivación de la película de protección no puede tener lugar. 
5. Corrosión electroquímica

\subsubsection{Corrosión localizada}

La corrosión localizada, en contraste, se concentra en pequeñas áreas de la superficie expuesta donde se forman agujeros o pequeños hoyos.

Las picaduras o pittings o son un proceso electroquímico en el cual pequeños huecos crecen rápidamente en la superficie de un metal bajo la influencia de un anión electrolítico y un campo eléctrico. El origen de los sitios de nucleación depende del número de factores de acuerdo al sistema. La técnica de $E N$ ha probado ser sensitiva para detectar cambios espontáneos en los procesos de corrosión llamados iniciación de picaduras y ataques de cavitación. Algunas estructuras microscópicas pueden inducir la preferencia al picado, como por ejemplo el acero inoxidable en solución con iones $\mathrm{Cl}^{-}$. Otras formas de corrosión incluyen corrosión intergranular, corrosión bajo esfuerzo, corrosión galvánica, etc (Callister, 2007).

La propagación inicial de la corrosión por picadura (pitting) en acero inoxidable en presencia de iones $\mathrm{Cl}^{-}$esta descrita en términos electroquímicos y pasos de corrosión. Por simplicidad, el proceso de picadura puede ser dividido en tres fases. En primer lugar, la nucleación de la picadura conduce a la formación de una pequeña área de picado, sin una superficie pasiva en el metal. En segundo lugar, el desarrollo de una picadura meta estable conduce a la disolución local de una parte significativa del metal subyacente. El resultado es la formación de un hoyo estable o repasivación. Posteriormente, en el tercer paso, el crecimiento de un pozo estable conduce a un daño en el metal y a la propagación de la picadura (Jirarungsatian y Prateepasen, 2010).

La nucleación, crecimiento y muerte de picaduras meta-estables produce transitorios de corriente con una duración del orden de unos pocos segundos. Las Picaduras meta-estables suelen consistir en un aumento de corriente lenta seguida de una fuerte caída de corriente (en el acero inoxidable) o por un aumento moderado de corriente seguido por una caída más lenta (en el acero al carbono y aleaciones de aluminio) (Cottis, 2001).

Las picaduras meta estables en metales y aleaciones han sido estudiadas por muchos autores con el objetivo de comprender el mecanismo de la corrosión y encontrar un posible método que prediga las tendencias a las picaduras en los metales. Por ejemplo, Cheng y 
5. Corrosión electroquímica

col. (1998) estudiaron las fluctuaciones de potencial y corriente de $E N$ para $A 516$ - 70 y $16 \mathrm{Mn}$ del acero al carbono en solución $\mathrm{NaHCO}_{3}+\mathrm{NaCl}$. Los autores sugieren que el rápido ascenso y la lenta recuperación de las fluctuaciones en la corriente reflejan el inicio, crecimiento y repasivación de picados meta estables. Mientras que las posibles fluctuaciones de potencial reflejan la respuesta de la capacidad del electrodo al crecimiento de picaduras.

La corrosión por picaduras meta estables de acero al carbono en solución alcalina carbonatada en presencia de iones $\mathrm{Cl}^{-}$ha prestado mucha atención. Bertocci y Ye (1984) analizaron las fluctuaciones de corriente en ausencia y presencia de iones $\mathrm{Cl}^{-}$. Concluyeron que los iones $\mathrm{Cl}^{-}$podrían aumentar la probabilidad de ruptura local de la película pasiva. Cheng y col. (1998) estudiaron la corrosión por picadura en el acero al carbono en solución con iones $\mathrm{Cl}^{-}$y encontraron que la velocidad de iniciación de picaduras se incrementa al comienzo de la inmersión. Después se alcanzo un máximo, seguido de una disminución con el tiempo de la tasa de iniciación debido al agotamiento de sitios activos. Muniandy y col. (2011) analizaron el $E N$ de potencial y corriente proveniente de la corrosión de acero al carbono (ASTM A106) en agua destilada usando análisis multifractal, encontrando diferentes etapas de corrosión del sistema.

\subsection{Metodología}

El metal estudiado fue acero API X52, el cual es un material comúnmente utilizado en las tuberías que transportan hidrocarburos. El uso de este material, en condiciones de trabajo, corresponde a emulsiones del tipo agua-aceite (hidrocarburo) con elevada solubilidad de gases corrosivos $\left(\mathrm{H}_{2} \mathrm{~S}, \mathrm{CO}_{2}\right.$ y $\left.\mathrm{O}_{2}\right)$ en la fase acuosa del hidrocarburo. Dichas características contribuyen a la intensificación del proceso de corrosión (Vasquez y col., 2003).

\section{Celda electroquímica}

El experimento se llevó a cabo en una celda de dos electrodos idénticos, que constan de discos de tubería de acero $A P I X 52$ con una superficie de $0.50 \mathrm{~cm}^{2}(\varnothing=0.8 \mathrm{~cm})$. Los discos de acero fueron puestos en una base de Nailamin integrados con resina de poliéster. 
5. Corrosión electroquímica

Las mediciones de ruido en corriente y voltaje se llevaron a cabo en dos electrodos idénticos (electrodos de trabajo). La corriente que fluye entre los dos electrodos de disco de acero, así como el potencial entre el electrodo de disco de acero y un electrodo de referencia son monitoreados.

Se utilizó un electrodo de referencia $(R E)$ de $\mathrm{Ag} / \mathrm{AgCl}$ en una solución saturada de $\mathrm{KCl}$. Los electrodos de trabajo fueron acero API X52 con una composición química de $0.06 \%$ C, $0.3 \%$ Si, $1.05 \%$ Mn, $0.002 \%$ S, $0.013 \%$ P, $0.25 \%$ Cu, $0.02 \%$ Ni, $0.02 \%$ Cr, $0.008 \%$ Mo, $0.05 \% \mathrm{~V}, 0.05 \% \mathrm{Nb}, 0.02 \% \mathrm{Ti}$ y Fe como equilibrio. Las superficies de los electrodos de trabajo fueron mecánicamente pulidas con papel de $\mathrm{SiC}$ (grado 400 y 600), y posteriormente con una pasta de grafito de $0.05 \mu$. La superficie se enjuagó con alcohol etílico. Los electrodos fueron usados inmediatamente después de su preparación.

\section{Medio electrolítico}

La solución base utilizada en el experimento fue $\mathrm{NaHCO}_{3}$ 0.5M. Además se añadió solución $\mathrm{NaCl} 0.2 \mathrm{M}$ a la solución base para producir dos soluciones $0.1 \mathrm{M}$ con iones $\mathrm{Cl}^{-}$. Las soluciones fueron preparadas utilizando reactivos de grado analítico y agua desionizada.

\section{Caracterización electroquímica}

Las mediciones de $E N$ en corriente y voltaje fueron registradas con un equipo Solartron SI 1250 con interfaz electroquímica en auto resistencia cero del amperímetro $(Z R A)$ sistema de medición con una frecuencia de muestreo de 1 punto por segundo durante 10 horas. Las mediciones de EN se llevaron a cabo en una celda electroquímica que consta de un frasco de vidrio de $150 \mathrm{ml}$ y dos electrodos idénticos de tubería API X52. Todos los experimentos se realizaron a temperatura ambiente para el electrodo estático y rotativo. 
5. Corrosión electroquímica

\subsection{Resultados}

En esta Sección 5.3 se presentan los resultados obtenidos del análisis visual y de entropía aproximada de los espectros de ruido electroquímico en función del tiempo.

\subsubsection{Análisis visual del $E N$}

La información obtenida a partir de las señales de $E N$ se llevó a cabo por medio del análisis visual de los espectros de $E N$ en función del tiempo. Ya que el sistema bajo análisis es un sistema ampliamente conocido y estudiado, Sección 5.1. por lo cual nos permite de forma sencilla y rápida obtener información del sistema. Además, a través de este tipo de análisis se puede lograr una valoración cualitativa del estado electroquímico del mismo.
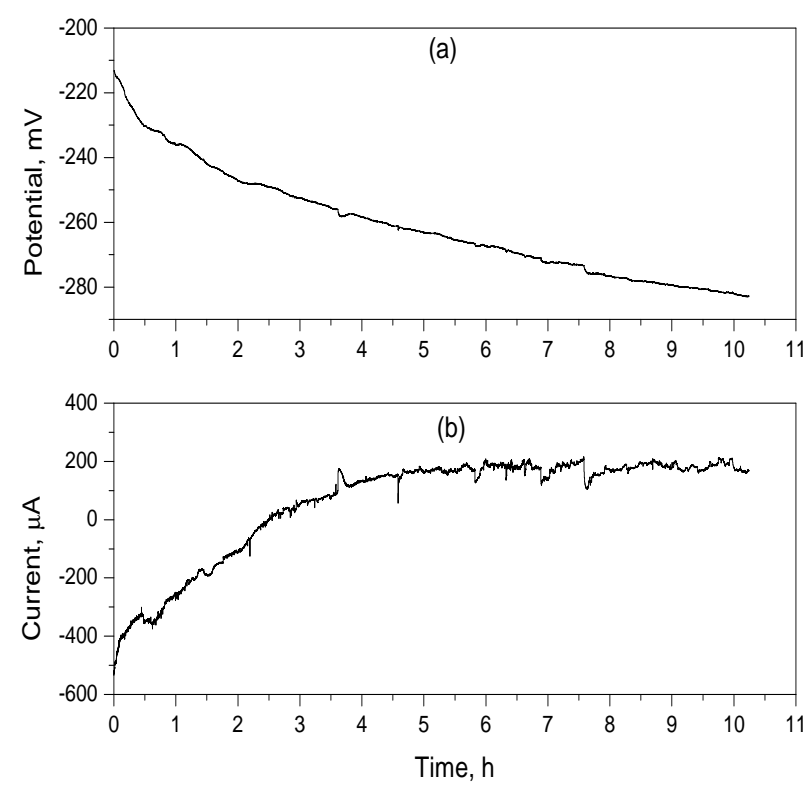

Figura 5.1. Serie de tiempo de (a) Voltaje y (b) Corriente del acero API X52 inmersa en solución acuosa $0.5 \mathrm{M} \mathrm{NaHCO}$ sin iones $\mathrm{Cl}^{-}$.

La Figura 5.1. a muestra las señales de $E N$ de voltaje del acero API X52 inmersa en solución acuosa $0.5 \mathrm{M} \mathrm{NaHCO}_{3}$, puede observarse un comportamiento monótono que 
5. Corrosión electroquímica

disminuye con el tiempo y no se observan transientes importantes en la señal. La serie de ruido de corriente para la solución de bicarbonatos sin iones $\mathrm{Cl}^{-}$(Figura 5.1.b) aumenta ligeramente y muestra fluctuaciones muy frecuentes de baja amplitud $(2-5 \mu \mathrm{A})$, presentando además transientes a partir de $\sim 3.5$ horas. La señal de $E N$ de voltaje del acero API X52 inmersa en solución acuosa 0.5M $\mathrm{NaHCO}_{3}$ sin iones $\mathrm{Cl}^{-}$(Figura 5.1. a) no muestra fluctuaciones importantes y disminuyen con el tiempo.
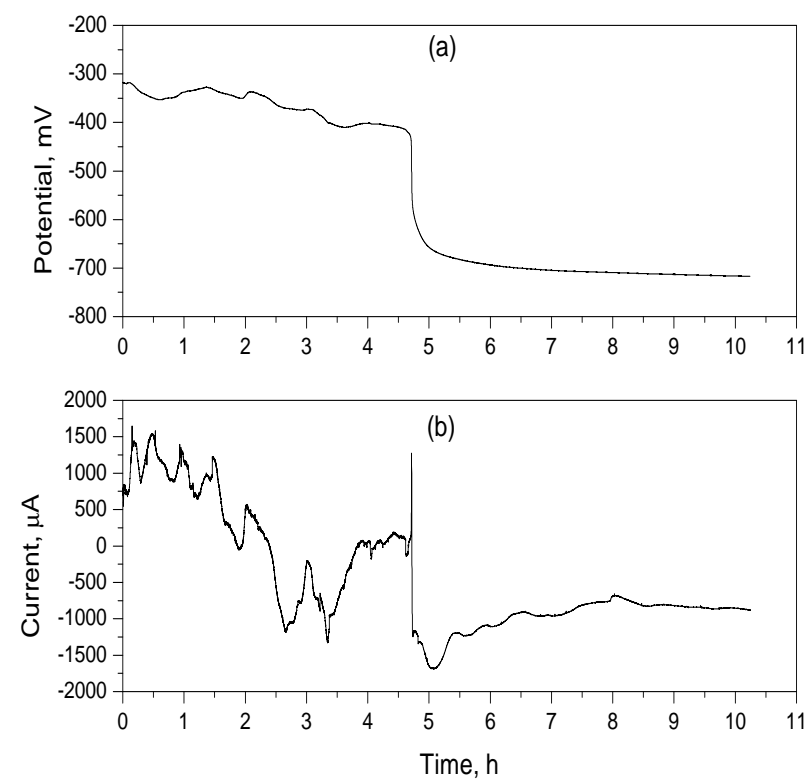

Figura 5.2. Serie de tiempo de (a) Voltaje y (b) Corriente del acero API X52 inmersa en solución acuosa $0.5 \mathrm{M} \mathrm{NaHCO}$ con iones $\mathrm{Cl}^{-}$a una concentración $0.1 \mathrm{M}$.

En la Figura 5.2 se muestran las series de tiempo de $E N$ de voltaje y corriente del acero API X52 inmersa en solución acuosa $0.5 \mathrm{M} \mathrm{NaHCO} 3$ con iones $\mathrm{Cl}^{-}$a una concentración 0.1M. Puede observarse que cuando se adiciona iones $\mathrm{Cl}^{-}$a la solución de bicarbonatos, se presenta una caída súbita en el potencial a 4.8 horas aproximadamente sin transientes importantes en la señal (Figura 5.2.a). Mientras que en la señal de corriente (Figura 5.2.b) se observa que las fluctuaciones de corriente registradas durante las primeras 5 horas además de ser de mayor magnitud (intensidad) tienen un patrón característico de 
5. Corrosión electroquímica

corrosión localizada (Uruchurtu y Dawson, 1987; Uruchurtu y Malo, 1997; Cottis y col., 2001). Este comportamiento pudiera ser debido a las presencia de iones $\mathrm{Cl}^{-}$en el sistema, los cuales aunque en baja concentración, podrían llegar con mayor facilidad a la interfase metal/electrólito. Lo cual contribuye a aumentar el coeficiente de transferencia de masa de estos aniones, disminuyendo por tanto la resistencia difusional y favoreciendo el proceso de la corrosión localizada por picaduras.

\subsubsection{Análisis de entropía aproximada}

Para cada corrida, los datos experimentales de $E N$ consistieron de 36864 observaciones. El tamaño de la ventana deslizante fue seleccionado como 540 observaciones, fijándose 21 escalas para realizar el análisis.

\section{Acero $\boldsymbol{A P I} X 52$ sin iones $C l^{-}$}

La Figura 5.3 muestra los patrones de entropía y su respectiva señal de $E N$ de voltaje del acero API X52 inmerso en solución acuosa $0.5 \mathrm{M} \mathrm{NaHCO}$. A simple vista la señal de $E N$ de voltaje no presentan transientes o fluctuaciones importantes. Sin embargo el análisis de entropía proporciona información a cerca de dos secciones donde ocurren cambios importantes:

Sección 1 En esta sección se registran los mayores valores de entropía, durante las primeras horas, los cuales pueden asociarse a la iniciación de picados meta estables en el sistema.

Sección 2 Puede observase que los valores de entropía presentan fluctuaciones indicando una posible formación y rompimiento local de la película pasiva formada sobre el sistema.

En la Figura 5.4 se observa la señal de $E N$ de corriente del acero API X52 inmersa en solución acuosa $0.5 \mathrm{M} \mathrm{NaHCO}$ y sus patrones de entropía. La serie de corriente para la solución de bicarbonatos sin iones $\mathrm{Cl}^{-}$(Figura 5.4 a) presenta un comportamiento que aumenta monotónicamente a través del tiempo. Observándose fluctuaciones muy frecuentes 
5. Corrosión electroquímica
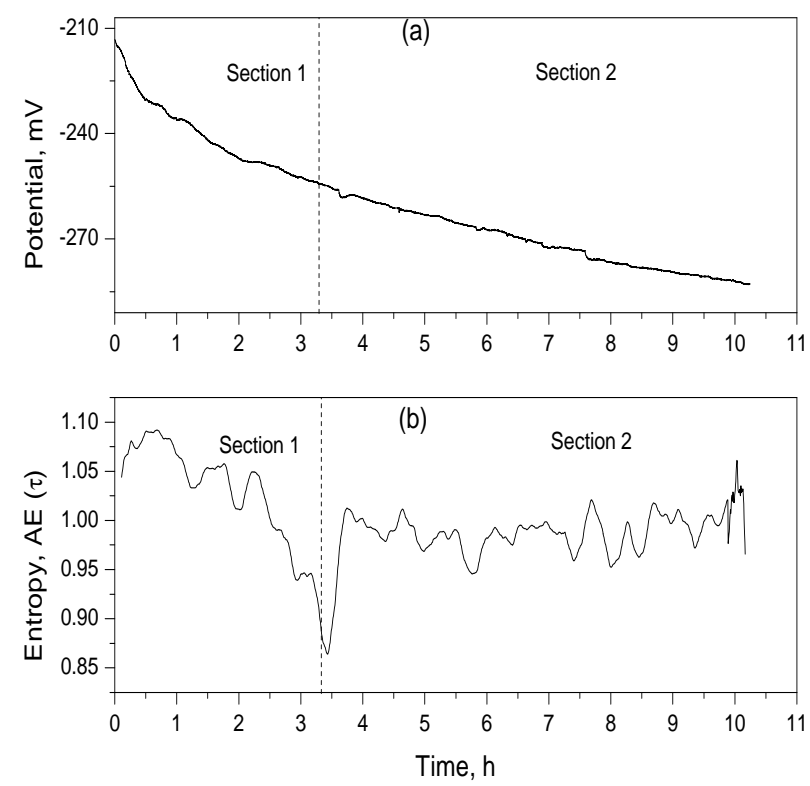

Figura 5.3. (a) Serie de tiempo de Voltaje y (b) Patrones de entropía $A p E n(\tau)$ de la serie de $E N$ de voltaje del acero API X52 inmersa en solución acuosa 0.5M $\mathrm{NaHCO}_{3}$.

de baja amplitud $(2-5 \mu \mathrm{A})$ durante las primeras horas y mayores transientes en la corriente después de $\sim 3.5$ horas. En la Figura 5.4.b puede observarse lo siguiente:

Sección 1 Se obtienen los mayores valores de entropía durante las primeras horas, los cuales pueden asociarse con la formación de picados meta estables en el sistema.

Sección 2 Se observan valores mínimos en esta sección, asociados al rompimiento y repasivación local de la película pasiva de la picadura formada a tiempos muy cortos.

La entropía es un concepto empleado para cuantificar la incertidumbre o irregularidades de un sistema dinámico. En general, la complejidad de un sistema real no es considerada a una solo escala. De esta forma, la entropía es dependiente de la escala, es decir, una señal es más regular para ciertas escalas de tiempo y mas irregular para otras. Es por ello que se analizaron las señales de ruido $E N$ empleando el enfoque a multiescala. Los resultados obtenidos se muestran en la Figura 5.5. Puede observarse que en la Figura 5.5a para escalas 
5. Corrosión electroquímica
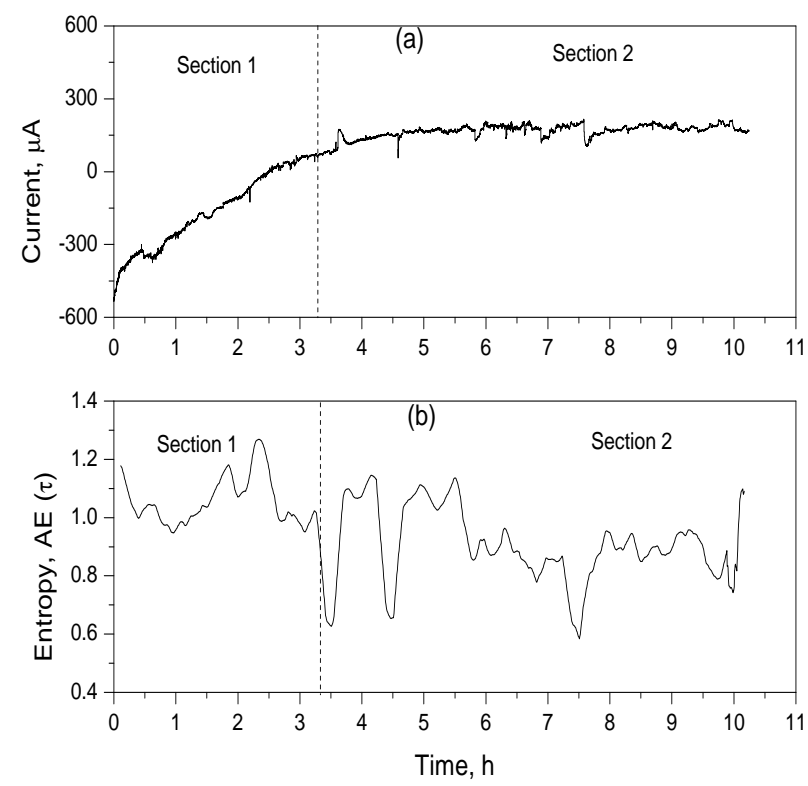

Figura 5.4. (a) Serie de tiempo de Corriente y (b) Patrones de entropía $A p E n(\tau)$ para la señal de $E N$ de corriente del acero $A P I X 52$ inmersa en solución acuosa $0.5 \mathrm{M} \mathrm{NaHCO}_{3}$.

bajas de tiempo se presentan valores altos de entropía asociados irregularidades en la señal. Sin embargo, a escalas $>0.015 \mathrm{seg}$ se muestran dos patrones correspondientes a valores bajos de entropía $\approx 3.5$ y 7.5 horas. Los cuales pudieran explicarse como la formación de picados meta estables que aparentemente se forman y posteriormente se destruyen con el tiempo.

Por otra parte, la Figura $5.5 \mathrm{~b}$ muestra los valores de entropía de la señal de corriente. Pueden observarse patrones de baja entropía a bajas escalas $\approx 3.5,4.5$ y 7.5, dos ellos concuerdan con los observados con la señal de voltaje (Figura 5.5a). Lo anterior, puede interpretarse como uniformidades en la señal relacionados con bajos valores de entropía, los cuales son mas evidentes a altas escalas de tiempo. En términos de corrosión se podría explicar que cuando se presentan los valores más bajos de entropía $\approx 3.5,4.5$ y 7.5, se esta formando la repasivación del metal y posteriormente se lleva a cabo el rompimiento, los cuales son asociados con altos valores de entropía. 


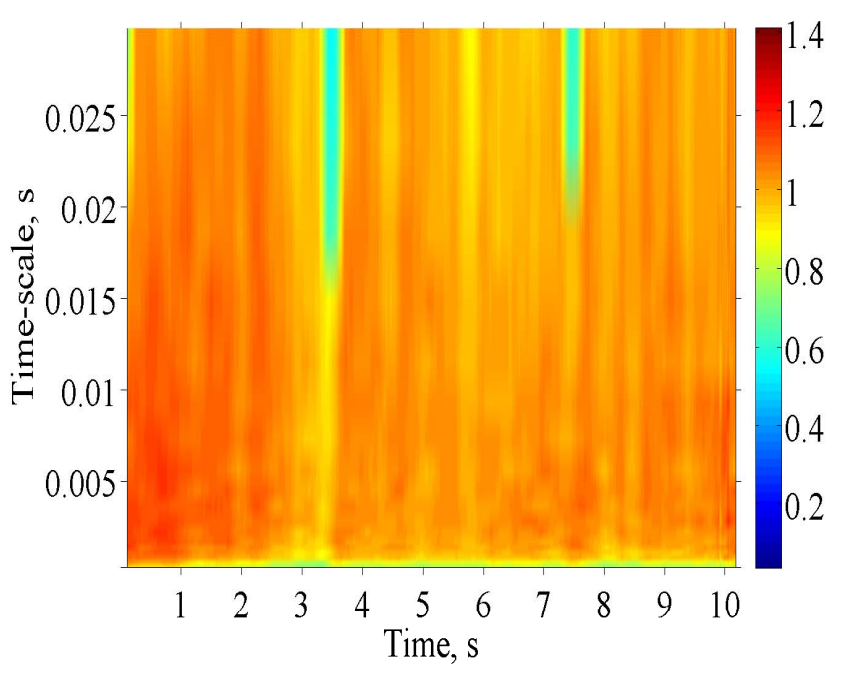

(a)

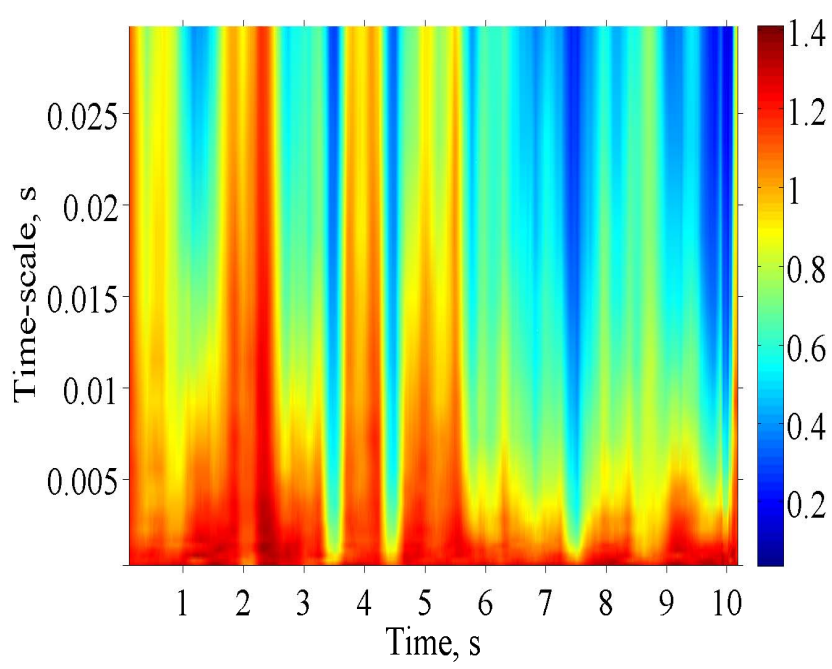

(b)

Figura 5.5. Valores de entropía a diferentes escalas de tiempo para la señal de $E N$ de (a) Voltaje y (b) Corriente del acero $A P I X 52$ inmersa en solución acuosa $0.5 \mathrm{M} \mathrm{NaHCO}$.

\section{Acero $\boldsymbol{A P I} X 52$ con iones $\mathrm{Cl}^{-}$}

En la Figura 5.6 se observa la serie de tiempo de voltaje del acero API X52 inmersa en solución acuosa $0.5 \mathrm{M} \mathrm{NaHCO}$ con iones $\mathrm{Cl}^{-}$a una concentración $0.1 \mathrm{M}$ y su patrón de entropía. Una caída súbita de potencial se observa a partir de $\sim 4.3$ horas en la solución 
5. Corrosión electroquímica
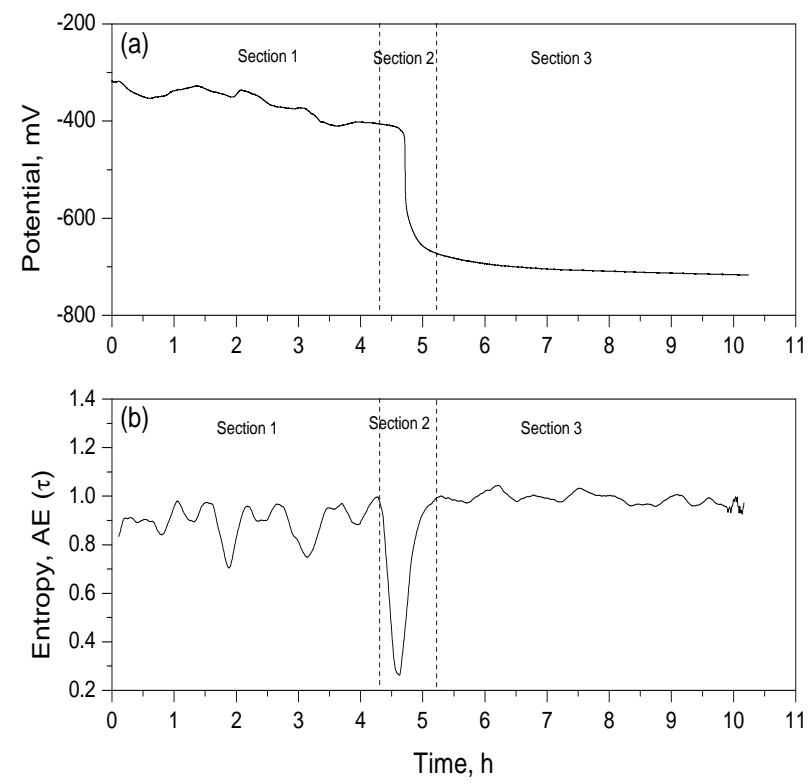

Figura 5.6. (a) Serie de tiempo de Voltaje y (b) Patrones de entropía $A p E n(\tau)$ para serie de $E N$ de voltaje del acero $\mathrm{API} X 52$ inmersa en solución acuosa $0.5 \mathrm{M} \mathrm{NaHCO}_{3}$ con iones $\mathrm{Cl}^{-}$a una concentración $0.1 \mathrm{M}$.

de $0.1 \mathrm{M}$ con iones $\mathrm{Cl}^{-}$(Figura 5.6. a). Mientras que el patrón de entropía muestra un valor mínimo (Sección 2) indicando un posible rompimiento local de la película pasiva formado sobre el acero. Mientras que en la Sección 3 no se muestran fluctuaciones de valores de entropía.

La serie de EN de corriente del acero API X52 inmersa en solución acuosa 0.5M $\mathrm{NaHCO}_{3}$ con iones $\mathrm{Cl}^{-}$a una concentración $0.1 \mathrm{M}$ y sus patrones de entropía se muestran en la Figura 5.7. La serie de corriente que se presenta en solución con iones $\mathrm{Cl}^{-}$(Figura 5.7.a) presenta oscilaciones entre $120-680 \mu \mathrm{A}$ durante las primeras 5 horas de inmersión. Después de este tiempo se observan fluctuaciones menores y un decaimiento de la corriente a valores por debajo al obtenido en la solución de bicarbonato sin iones $\mathrm{Cl}^{-}$. En la Figura 5.7.b se divide en tres secciones:

Sección 1 Muestra oscilaciones durante las primeras horas, las cuales pueden asociarse a la 
5. Corrosión electroquímica
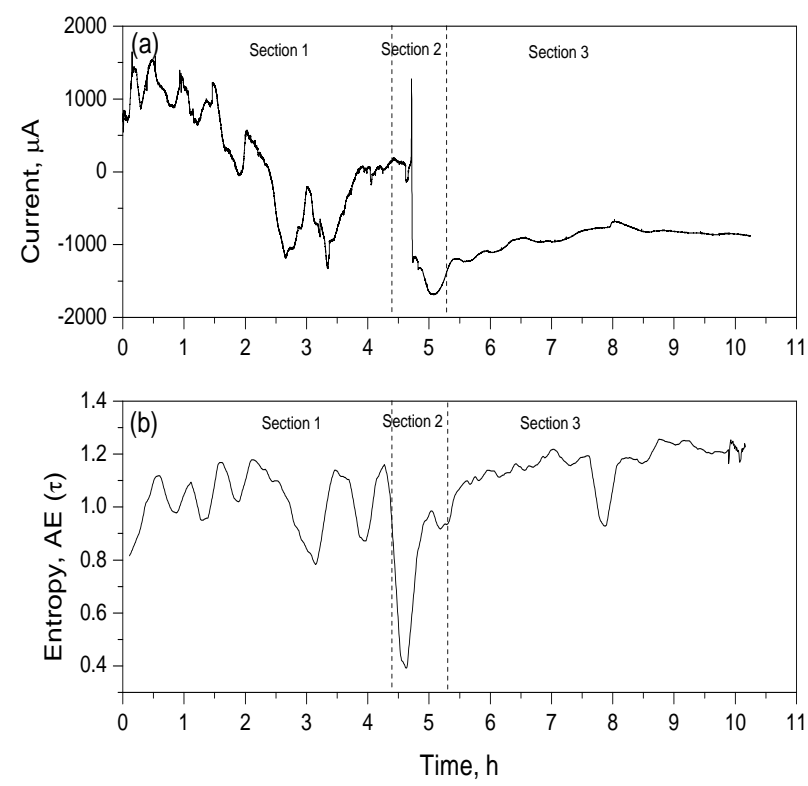

Figura 5.7. (a) Serie de tiempo de Corriente y (b) Patrones de entropía para la señal de EN de corriente del acero $\mathrm{API} X 52$ inmersa en solución acuosa $0.5 \mathrm{M} \mathrm{NaHCO}$ con iones $\mathrm{Cl}^{-}$a una concentración $0.1 \mathrm{M}$.

iniciación de picados meta estables.

Sección 2 Se observa una disminución súbita de la corriente indicando un posible rompimiento local de la película pasiva formada sobre el acero.

Sección 3 Disminución de la corriente al valor promedio original se debe a una repasivación de la picadura formada a tiempos muy cortos.

En las señales de $E N$ de corriente puede observarse transientes a través del tiempo, los cuales pueden ser asociados a diferentes mecanismos de corrosión. En estudios de $E N$ de aceros inmersos en carbonatos, las fluctuaciones o transientes de ruido de potencial y corriente indican la iniciación de picados meta estables. Donde aumentos súbitos de potencial y su correspondiente disminución súbita de la corriente indican el rompimiento local de la película pasiva formada sobre el acero. Y la disminución de la corriente al valor promedio original se debe a una repasivación de la picadura formada a tiempos muy cortos. 


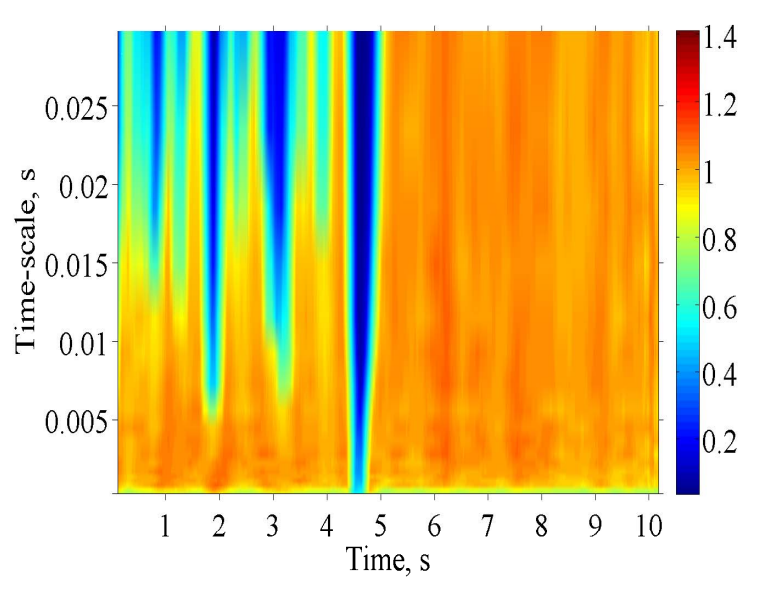

(a)

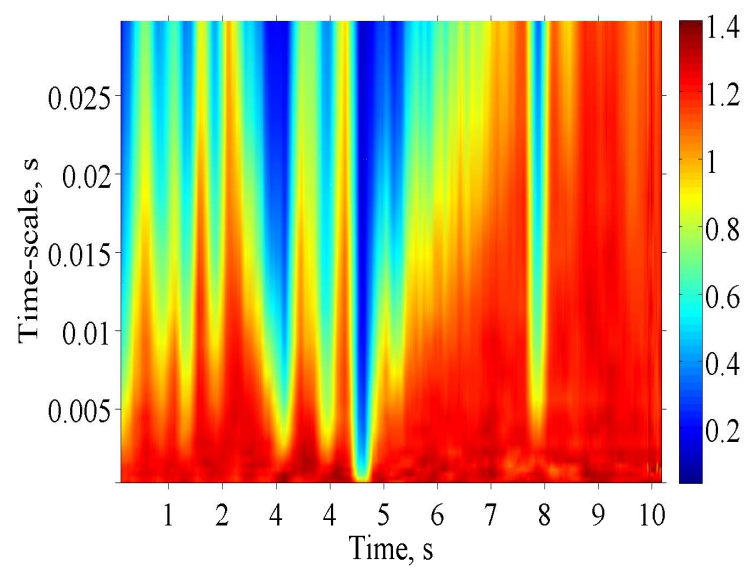

(b)

Figura 5.8. Valores de entropía a diferentes escalas de tiempo para la señal de $E N$ de (a) Voltaje y (b) Corriente del acero $A P I X 52$ inmerso en solución acuosa $0.5 \mathrm{M} \mathrm{NaHCO}$ con iones $\mathrm{Cl}^{-}$a una concentración $0.1 \mathrm{M}$.

Mientras que el potencial de una interfase metal-solución indica cambios en el estado termodinámico de la superficie (fenómenos de adsorción interfacial de iones, estado pasivo y estado activo de la superficie) la corriente tiene que ver con la cinética o velocidad de pérdida de metal originado por un proceso de corrosión.

Por otra parte se realizó el análisis de entropía multiescala para obtener información del 
5. Corrosión electroquímica

mecanismo de corrosión de la señal a distintas escalas de tiempo. La Figura 5.8 muestra los valores de entropía de las señales de voltaje y corriente del acero API X52 inmerso en solución acuosa 0.5M $\mathrm{NaHCO}_{3}$ con iones $\mathrm{Cl}^{-}$. Observe la Figura 5.8a, a bajas escalas de tiempo solo es evidente la formación de un patrón de baja entropía. Sin embargo, a altas escalas de tiempo se presentan cuatro patrones de baja entropía ( $\approx 0.5,1.5,2.8$ y 4.0 horas), los cuales pueden reflejar uniformidades en la señal de voltaje.

Además la Figura 5.8 b también proporciona información útil de la dinámica de corrosión. Esto es, pueden observarse que a altas escalas de tiempo se presentan varios patrones de bajos de entropía $(\approx 0.5,1.5,2.0,2.5,3.8,4.0$ y 7.5 horas $)$, cuatro ellos concuerdan con los obtenidos con la señal de voltaje (Figura 5.8a).

\subsection{Conclusiones}

La información obtenida a partir de las señales de $E N$ de corriente y voltaje del acero API X52 inmerso en solución acuosa $0.5 \mathrm{M} \mathrm{NaHCO}$ con y sin iones $\mathrm{Cl}^{-}$permite obtener información del comportamiento de pasos de mecanismos de corrosión.

En el caso del análisis visual de los espectros de ruido electroquímico en función del tiempo suele ser la forma más sencilla y rápida de obtener información del sistema y, a través de este, se puede lograr una valoración cualitativa del estado electroquímico del mismo. Sin embargo su principal desventaja es que se requiere de un suficiente conocimiento del sistema investigado para poder interpretar las señales de EN. Por otra parte, a partir de los resultados obtenidos en el análisis de entropía aproximada se concluye que este método puede proporcionar información útil en la caracterización de los mecanismo de corrosión. La idea principal es que zonas de baja entropía son asociados a uniformidades en la señal y valores altos de entropía se relacionan a fluctuaciones o irregularidades. Por ejemplo, en la señal de $E N$ de corriente y voltaje del acero $A P I X 52$ sin iones $\mathrm{Cl}^{-}$se identificaron a bajas escalas $\mathrm{a} \approx 3.5,4.5$ y $7.5 \mathrm{seg}$ zonas de baja entropía que pueden asociarse con la repasivación del metal. En el caso de las señales de EN de corriente y voltaje del acero API X52 con iones $\mathrm{Cl}^{-}$se observó que a bajas escalas de tiempo se encuentran varias zonas de baja entropía 
siendo $\mathrm{a} \approx 3.5,4.5$ y $7.5 \mathrm{seg}$ las más evidentes. Lo anterior se interpreta que debido a la presencia de iones $\mathrm{Cl}^{-}$se acelera el mecanismo de corrosión de forma tal que se forma la repasivación y posteriormente el rompimiento de la película protectora en periodos cortos de tiempo. Por lo cual se concluye que, el análisis de entropía aproximada permite obtener información acerca de los mecanismos de corrosión (corrosión localizada, corrosión uniforme, etc.) a partir de las señales de ruido electroquímico de sistemas dinámicos. 


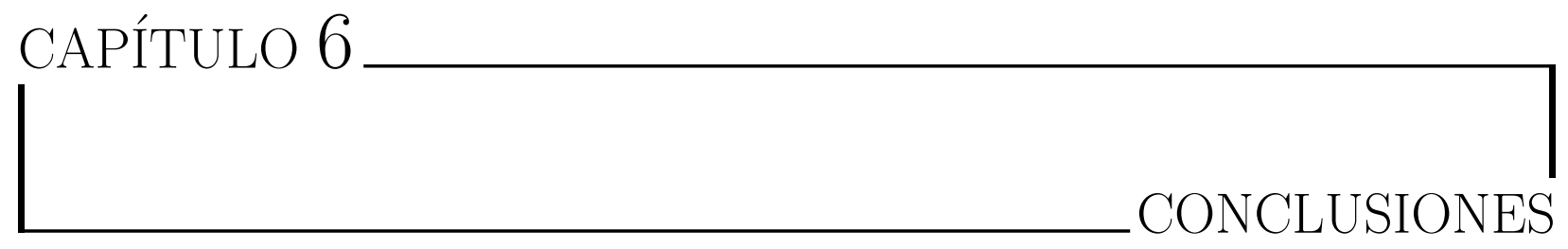

En este trabajo de investigación se empleó el análisis de entropía aproximada como herramienta durante el monitoreo de columnas de destilación y sistemas de corrosión electroquímica. Con el propósito de identificar inestabilidades o irregularidades como consecuencia de perturbaciones, fallas de operación, formaciones de picaduras, pasivación o pérdida de metal en los procesos analizados. Los resultados se dividen en dos partes principales, los cuales se describen a continuación:

- En la Parte I se presentó todo lo relacionado con el análisis de señales sintéticas $1 / f^{\beta}$ y de difracción de rayos $X$ como parte de la validación de los métodos propuestos en la investigación. En el Capítulo 2 se analizaron señales sintéticas $1 / f^{\beta}$ que abarcan señales desde el ruido blanco hasta de movimiento browniano. Se utilizaron este tipo de señales debido a que muchos sistemas que presentan características fractales pueden ser caracterizadas a través de estas. Los resultados obtenidos demostraron que las técnicas de entropía propuestas en esta investigación pueden detectar correlaciones o patrones estructurados en series temporales considerando efectos de tendencia a diferentes escalas de tiempo.

- En el Capítulo 3 se analizaron señales reales de difracción de rayos $X$ con ruido intenso, con la idea de detectar regiones angulares donde las fluctuaciones de intensidad de 
difracción de rayos $X$ no son aleatorias del todo, sino que exhiben cierto grado de regularidad. Para lograrlo, regularidades en las señales de intensidad se cuantificaron mediante un análisis de entropía aproximada multiescala que fueron asociados con patrones geométricos contenidos en la compleja estructura del material. Con lo anterior se demostró que las técnicas de entropía propuestas en esta investigación permiten obtener información de señales aparentemente aleatorias, en presencia de ruido y con tendencia.

- En la Parte II de la tesis se presentó todo lo relacionado con el monitoreo de columnas de destilación y sistemas de corrosión electroquímica. En el Capítulo 4 se presentaron los resultados obtenidos del monitoreo de columnas de destilación binarias por medio de los análisis de entropía aproximada. La idea principal para interpretar los resultados de entropía fueron: altos valores de entropía se asociaron a cambios o perturbaciones (irregularidades o fluctuaciones) en la señal monitoreada como consecuencia de cambios de operación en las variables manipuladas (ejem., relación de reflujo y flujo de vapor) o perturbaciones (ejem., alimentación). Mientras que bajos valores de entropía (patrones estructurados) correspondieron a situaciones normales de operación donde se presentan estructuras regulares, que corresponde a un sistema en operación normal en ausencia de irregularidades o cambios abruptos en la señal de proceso. Los resultados obtenidos en este capítulo demostraron que el análisis de entropía aproximada puede ser utilizado como sistema de monitoreo. Debido a que tiene la capacidad de detectar patrones o irregularidades contenidos en las series temporales de las columnas de destilación.

- Finalmente en el Capítulo 5 se presentó todo lo relacionado con el análisis realizado a señales de $E N$ de corriente y voltaje del acero API X52 inmerso en solución acuosa $0.5 \mathrm{M} \mathrm{NaHCO} 3$ con y sin iones $\mathrm{Cl}^{-}$. De los resultados obtenidos de los análisis de entropía se concluye que, se obtiene información acerca de los mecanismos de corrosión (corrosión localizada, uniforme, etc.) a partir de señales $E N$ de un sistema dinámico.

Cabe agregar que la metodología expuesta en este trabajo de investigación puede extenderse a otros casos de estudio de mayor complejidad. A través de la presente tesis se ha 
enfatizado que las técnicas de entropía (Apéndices $\mathrm{B} / \mathrm{C} \mid \mathrm{D}$ ) propuestas en esta investigación permiten obtener información de señales temporales que son aparentemente aleatorias, y que además se consideran efectos de tendencia y ruido. 
Alvarez-Ramirez, J. y Rodriguez, E., 2011. "Long-term recurrence patterns in the late 2000 economic crisis: Evidences from entropy analysis of the Dow Jones index", Technological Forecasting and Social Change, Vol. 78, No. 8, 1332 - 1344.

Bakshi, B.R., 1998. "Multiscale PCA with application to multivariate statistical process monitoring", AIChE Journal, Vol. 44, No. 7, 1596 - 1610.

Barnard, G.A., 1959. "Control Charts and Stochastic Processes", Journal of the Royal Statistical Society B, Vol. 21, 239 - 271.

Bertocci, U. y Ye, Y.X., 1984. "An examination of current fluctuations during pit initiation in Fe-Cr alloys", J. Electrochem. Soc., Vol. 131, $1011-1017$.

Callister, W.D., 2007. Introducción a la ciencia e ingeniería de los materiales. Limusa Wiley, 2 edición.

Camesasca, M., Kaufman, M., y Manas-Zloczower, I., 2006. "Quantifying Fluid Mixing with the Shannon Entropy", Macromolecular Theory and Simulations, Vol. 15, No. 8, 595 607.

Castañeda, I., Romero, M., Malo, J., y Uruchurtu, J., 2010. "Ruido electroquímico de la 
erosión-corrosión en cobre: su relación con los parámetros hidrodinámicos", Revista de Metalurgia, Vol. 46, No. 5.

Chang, C.C. y Yang, R.J., 2006. "A particle tracking method for analyzing chaotic electroosmotic flow mixing in 3D microchannels with patterned charged surfaces", Journal of Micromechanics and Microengineering, Vol. 16, No. 8, 1453 - 1462.

Cheng, Y.F., Rairdan, B., y Luo, J.L., 1998. "Features of electrochemical noise generated during pitting of inhibited A516-70 carbon steel in chloride solutions", Journal of Applied Electrochemistry, Vol. 28.

Costa, M., Goldberger, A.L., y Peng, C.K., 2002. "Multiscale Entropy Analysis of Complex Physiologic Time Series", Phys. Rev. Lett., Vol. 89, No. 6, 068102.

Cottis, R.A., 2001. "Interpretation of Electrochemical Noise Data", Corrosion, Vol. 57, No. $3,265-285$.

Cottis, R.A., Al-Awadhi, M.A., Al-Mazeedi, H., y Turgoose, S., 2001. "Measures for the detection of localized corrosion with electrochemical noise", Electrochimica Acta, Vol. 46, $3665-3674$.

Desborough, L. y Harris, T., 1993. "Performance assessment measures for univariate feedforward/feedback control", Canadian J. Chemical Engineering, Vol. 71, No. 4, 605 $-616$.

Doherty, M.F. y Perkins, J.D., 1982. "On the dynamics of distillation processes IV: Uniqueness and stability of the steady-state in homogeneous continuous distillations", Chemical Engineering Science, Vol. 37, No. 4, 381 - 392.

Dorfman, J. R., 1999. An introduction to chaos in nonequilibrium statistical mechanics. Cambridge university press.

Eden, D.A., John, D.G., y Dawson, J.L. "Corrosion Monitoring". US Patent No. 5139627, 1992. 
Garces-Rosas, S.M., Diciembre 1999. Propuesta de un sistema Automático para Detección y Diagnóstico de Fallas. Tesis Doctoral, Universidad de los Andes. Mérida, Venezuela.

Gardner, M., 1978. "Mathematical games: White and brown music, fractal curves, and one-over-f noise", Scientific American, Vol. 238, No. 4, 16 - 32.

Gates, B. C., Katzer, J. R., y Schuit, G.C.A., 1979. Chemistry of catalytic processes. McGrawHill, New York.

Goellner, J., 2004. "Elektrochemisches Rauschen bei der Korrosion", Werkstoffe und Korrosion, Vol. 55, 727 - 734 .

Goldberger, A.L., Peng, C.K., y Lipsitz, L.A., 2002. "What is physiologic complexity and how does it change with aging and disease?", Neurobiology of Aging, Vol. 23, No. 1, 23 26.

Guillen, P., Paredes, M., y Camacho, O., 2005. "A proposal method for fault detection and diagnosis in chemical processes instrumentation using wavelet transform", Revista Técnica de la Facultad de Ingeniería. Universidad del Zulia, Vol. 28, No. 1, 68-77.

Gupta, S., Ray, S., y Samanta, A.N., 2009. "Nonlinear control of debutanizer column using profile position observer", Computers and Chemical Engineering, Vol. 33, No. 6, $1202-$ 1211.

Halvorsen, I.J. y Skogestad, S. "Theory Of Distillation". En Wilson, Ian D., editor, Encyclopedia of Separation Science, páginas 1117 - 1134. Academic Press, Oxford, 2000.

Harris, T.J., Seppala, C.T., y Desborough, L.D., 1999. "A review of performance monitoring and assessment techniques for univariate and multivariate control systems", Journal of Process Control, Vol. 9, No. 1, 1 - 17.

Hassan, M., Terrien, J., Marque, C., y Karlsson, B., 2011. "Comparison between approximate entropy, correntropy and time reversibility: Application to uterine electromyogram signals", Medical Engineering and Physics, Vol. 33, No. 8, 980 - 986. 
Bibliografía

Hornero, R., Aboy, M., Abasolo, D., McNames, J., y Goldstein, B., 2005. "Interpretation of approximate entropy: analysis of intracranial pressure approximate entropy during acute intracranial hypertension", IEEE Transactions on Biomedical Engineering, Vol. 52, 1671 $-1680$.

Jirarungsatian, C. y Prateepasen, A., 2010. "Pitting and uniform corrosion source recognition using acoustic emission parameters", Corrosion Science, Vol. 52, No. 1, 187 - 197.

Jáuregui-Correa, J.C. y Gonzalez-Brambila, O.M., 2010. Mechanical Vibrations of Discontinuous Systems. Nova Science Publishers Inc., 1 edición.

Kane, R.D., 2007. "A new approach to corrosion monitoring", Chem.Eng., Vol. 114, $34-41$.

Kuzovlev, Yu.E., 2012. "Quantum Brownian motion and a theorem on fundamental 1/f noise".

Liu, Q., Wang, A., Wang, X., Gao, P., y Zhang, T., 2008. "Synthesis, characterization and catalytic applications of mesoporous gamma-alumina from boehmite sol.", Microporous and Mesoporous Materials, Vol. 111, 323 - 333.

Luyben, W., 1996. Process Modeling, Simulation and Control for Chemical Engineers. McGraw Hill International Editions, second edición.

Martina, E., Rodriguez, E., Escarela-Perez, R., y Alvarez-Ramirez, J., 2011. "Multiscale entropy analysis of crude oil price dynamics", Energy Economics, Vol. 33, 936 - 947.

Milotti, E., 1996. "El ruido 1/f en Investigación y Ciencia", Investigación y ciencia, Vol. $243,66-71$.

Miramontes, P., 1999. "El Color del Ruido", Ciencias, Vol. 54, 4 - 15.

Morari, M. y E., Zafiriou, 1989. Robust Process Control. Prentice-Hall, New York.

Muniandy, S.V., Chew, W.X., y Kan, C.S., 2011. "Multifractal modelling of electrochemical noise in corrosion of carbon steel", Corrosion Science, Vol. 53, $188-200$. 
Bibliografía

nslijgenetics, 2011. "Center for Genomics and Human Genetics". URL www. nslij-genetics.org/wli/1fnoise.

Orantes, A., Kempowsky, T., y Lann, M.V. Le, 2006. "Classification as an aid tool for the selection of sensors used for fault detection and isolation", Transactions of the Institute of Measurement and Control, Vol. 28, No. 5, $457-479$.

Orantes, A., Kempowsky, T., Lann, M.V. Le, Prat, L., Elgue, S., Gourdon, C., y Cabassud, M., 2007. "Selection of Sensors by a New Methodology Coupling a Classification Technique and Entropy Criteria", Chemical Engineering Research and Design, Vol. 85, No. $6,825-838$.

Orantes, A., Kempowsky, T., Lann, M.V. Le, y Aguilar-Martin, J., 2008. "A new support methodology for the placement of sensors used for fault detection and diagnosis", Chemical Engineering and Processing: Process Intensification, Vol. 47, No. 3, 330 - 348. 10th French Congress on Chemical Engineering.

Page, E.S., 1954. "Continuous Inspection Schemes", Biometrika, Vol. 41, 100 - 114.

Pan, Y.H., Wang, Y.H., Liang, S.F., y Lee, K.T., 2011. "Fast computation of sample entropy and approximate entropy in biomedicine", Computer Methods and Programs in Biomedicine, Vol. 1, No. 0.

PhysioNet, 2011. "The research resource for complex physiologic signals". URL http: //www.physionet.org/physiobank/database/synthetic/tns/.

Pincus, S.M., 1991. "Approximate entropy as a measure of system complexity", Proc. Nati. Acad. Sci. USA, Vol. 88, $2297-2301$.

Pincus, S.M., 1995. "Approximate entropy (ApEn) as a complexity measure", Chaos, Vol. 5, No. 110.

Pincus, S.M., 2006. "Approximate entropy as a measure of irregularity for psychiatric serial metrics", Bipolar Disorders, Vol. 8, No. 5, $430-440$. 
Pincus, S.M., 2008. "Approximate Entropy as an Irregularity Measure for Financial Data", Econometric Reviews, Vol. 27, No. 4-6, 329 - 362.

Pincus, S.M. y Kalman, R.E., 2004. "Irregularity, volatility, risk and financial market time series", Proceedings of the National Academy of Sciences USA, Vol. 101, 13709 - 13714.

Pincus, S.M. y Singer, B.H., 1995. "Randomness and degrees of irregularity", Proceedings of the National Academy of Sciences USA, Vol. 93, 2083 - 2088.

Pérez-Canales, D., ÁlvarezRamírez, J., Jáuregui-Correa, J.C., Vela-Martínez, L., y HerreraRuiz, G., 2011. "Identification of dynamic instabilities in machining process using the approximate entropy method", International Journal of Machine Tools and Manufacture, Vol. 51, No. 6, $556-564$.

Pugjaner, L., Ollero, P., Prada, C., y Jiménez, L., 2006. Estrategias de modelado, simulación y optimización de procesos químicos. Sintesis.

Pugliese, E. y Castellano, C., 2009. "Heterogeneous pair approximation for voter models on networks", EPL (Europhysics Letters), Vol. 88, No. 5, $58004-58010$.

Richman, J.S. y Moorman, J.R., 2000. "Physiological time-series analysis using approximate entropy and sample entropy", American Journal of Physiology- Heart and Circulatory Physiology, Vol. 278, 2039 - 2049.

Roberts, S.W., 1959. "Control Chart Tests Based on Geometric Moving Averages", Technometrics, Vol. 1, $239-250$.

Rodriguez, I., 2005. "Implementación de un sistema dedicado al monitoreo de condiciones de proceso en una columna de destilación experimental", Grupo Exergia, Instituto Mexicano del Petróleo.

Seaborg, D.E., Edgar, T.F., y Mellichamp, D.A., 2004. Process Dynamics and Control. John Wiley, 2da edición. 
Bibliografía

Shannon, C.E., 1948. "A Mathematical Theory of communication", The Bell System Technical Journal, Vol. 27, 379 - 423.

Shewhart, W.A., 1926. "Quality Control Charts", The Bell System Technical Journal, Vol. $5,593-603$.

Shi, L., Zhang, Z.Y., y Yang, R., 2000. "Shannon Entropy Characteristics of Air-Water Two Phase Flow Regimes in Vertical Pipes", Chinese Journal of Nuclear Power Engineering, Vol. $5,411-415$.

Shi, L., Zhang, Z.Y., y Gao, Z.Y., 2004. "Shannon Entropy Characteristics of Twophase Flow Density Wave Instability Experiments for 200MW Nuclear Heating Reactor", Chinese Journal of Nuclear Power Engineering, Vol. 1, 18 - 21.

Skogestad, S., 1997. "Dynamics and Control of Distillation Columns - A Critical Survey", Modeling, Identification and Control, Vol. 18, No. 3, 177 - 217.

Skogestad, S. y Morari, M., 1988. "Understanding the dynamic behavior of distillation columns", Ind. Eng. Chem. Research, Vol. 27, No. 10, 1848 - 1862.

Sánchez, B., Enero 2002. Detección y Diagnóstico de Fallas utilizando Estructuras de Transición. Tesis Doctoral, Universidad de los Andes. Mérida, Venezuela.

Stoumbos, Z.G., Reynolds, M.R., Ryan, T.P., y Woodall, W.H., 2000. "The State of Statistical Process Control as We Proceed Into the 21st Century", Journal of the American Statistical Association, Vol. 95, 992 - 998.

Tarantino, R., Noviembre 1999. Detección de Fallas en Sistemas Dinámicos lineales variantes en el tiempo. Tesis Doctoral, Universidad de los Andes. Mérida, Venezuela.

Treybal, R.E., 1998. Operaciones de transferencia de masa. Mc Graw Hill, 2da. edición.

Uruchurtu, J. y Dawson, J.L., 1987. "Noise Analysis of Pure Aluminum under Different Pitting Conditions", Corrosion, Vol. 43, No. 1, $19-25$. 
Uruchurtu, J. y Malo, J.M., 1997. "Electrochemical Noise as a Powerful Electrochemical Technique for Corrosion Studies", Trends in Corrosion Research, Vol. 2, 49 - 58.

Valderas, J.M., Alba, J.M., y Olmedo, E., 2002. "Modelización estocástica en los mercados financieros: un puente entre lo simple y lo complejo", Encuentros Multidisciplinarios, Vol. $12,38-47$.

Vasquez, R., Hazan, L., Uruchurtu, J., Malo, J.M., y Genesca, J., 2003. "Corrosión y protección de un acero API-X52 en crudo de petróleo", Afinidad, Vol. 60, No. 504, 1363 143.

Vela-Martínez, L., Jáuregui-Correa, J.C., González-Brambila, O.M., Herrera-Ruiz, G., y Lozano-Guzmán, A., 2009. "Instability conditions due to structural nonlinea-rities in regenerative chatter", Nonlinear Dynamics, Vol. 56, 415 - 427.

Wang, J.A., Bokhimi, X., Morales, A., Novaro, O., Lopez, T., y Gomez, R., 1999. "Aluminum Local Environment and Defects in the Crystalline Structure of Sol-Gel Alumina Catalyst", The Journal of Physical Chemistry B, Vol. 103, No. 2, 299 - 303.

Wassermann, J., 2002. IASPEI New manual of seismological observatory practice, volumen 1-2. GeoForschungsZentrum Potsdam, 1 edición.

Yan, R. y Gao, R.X., 2007. "Approximate Entropy as a diagnostic tool for machine health monitoring", Mechanical Systems and Signal Processing, Vol. 21, No. 2, $824-839$.

Yurusoy, M., Yilbas, B.S., y Pakdemirli, M., 2006. "Non-Newtonian fluid flow in annular pipes and entropy generation: Temperature-dependent viscosity", Sadhana, Vol. 31, 683 695.

Zamarreño, J.M. y Rueda, A., 2003. "Comunicación de Labview con EcosimPro para la generación de predicciones en una columna de destilación". XXIV Jornadas de Automática. 
Zhang, Y.C., 1991. "Complexity and 1/f noise. A phase space approach", J. Phys. L. France, Vol. 1, No. 7, $971-977$.

Zhang, Z. y Shi, L., 1999. "Shannon entropy characteristics of two phase flow systems", J. Appl. Phys., Vol. 85, No. 11, $7544-7551$.

Zhong, W. y Zhang, M., 2005. "Characterization of dynamic behavior of a spout-fluid bed with Shannon entropy analysis", Powder Technology, Vol. 159, No. 23, 121 - 126.

Zhong, W.Q., Jin, B.S., Zhang, Y., Wang, X.F., Zhang, M.Y., y Xiao, R., 2009. "Shannon entropy increment analysis on dynamic behavior of a biomass fluidized bed", Journal of Physics: Conference Series, Vol. 147, No. 1, 012071. 


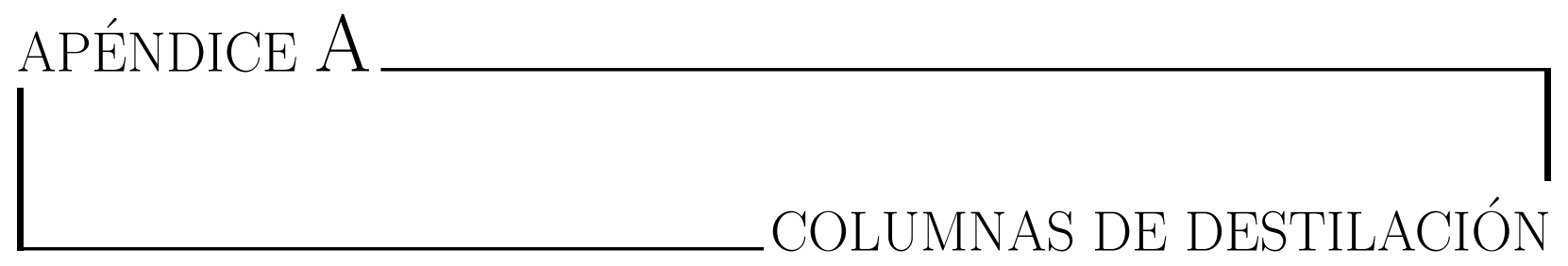

\section{A.1. Casos de estudio}

Los modelos de las columnas bajo estudio se obtienen a partir de la siguientes consideraciones (Doherty y Perkins, 1982; Halvorsen y Skogestad, 2000): flujos equimolares, presión constante, equilibrio termodinámico y mezclado perfecto en todos los platos, control de nivel perfecto en el rehervidor y el tanque de condensados, condensador total y masa despreciable de vapor en cada plato. 
A. Columnas de destilación

Tabla A.1. Datos en estado estacionario de la Columna A

\begin{tabular}{lc}
\hline Sistema & Volatilidad relativa constante $\alpha=1.5$ \\
\hline \hline Modelo Termodinámico & Ley de Raoult \\
Presión, atm & 1 \\
Composición de alimento, $C_{F}$ & 0.5 \\
Composición de destilado, $C_{D}$ & 0.99 \\
Composición de fondos, $C_{B}$ & 0.01 \\
Plato de alimentación $N_{F}(1)$ & 20 \\
N, número de platos ${ }^{(2)}$ & 39 \\
F, Kmol/min & 1 \\
V/F & 3.206 \\
R/F & 2.706 \\
Acumulado en condensados, Kmol & 0.5 \\
Acumulado en rehervidor, Kmol & 0.5 \\
Tiempo de residencia del condensador, min & 0.16 \\
Tiempo de residencia del rehervidor, min & 0.13 \\
Acumulado de plato, Kmol & 0.5 \\
Constante de tiempo de plato, min & 0.063 \\
& \\
\hline
\end{tabular}

(1) contando desde abajo

(2) sin incluir rehervidor y tanque de condensados 
A. Columnas de destilación

Tabla A.2. Datos en estado estacionario de la Columna B

\begin{tabular}{lc}
\hline Sistema & Benceno-Tolueno \\
\hline \hline Modelo Termodinámico & Ley de Raoult \\
Presión, atm & 1 \\
Composición de alimento, $C_{F}$ & 0.5 \\
Composición de destilado, $C_{D}$ & 0.99 \\
Composición de fondos, $C_{B}$ & 0.01 \\
Plato de alimentación $N_{F}(1)$ & 10 \\
N, número de platos ${ }^{(2)}$ & 18 \\
F, Kmol/min & 1.32 \\
V/F & 1.365 \\
R/F & 0.865 \\
Acumulado en condensados, Kmol & 32 \\
Acumulado en rehervidor, Kmol & 41.6 \\
Tiempo de residencia del condensador, min & 17.8 \\
Tiempo de residencia del rehervidor, min & 16.9 \\
Acumulado de plato, Kmol & 4 \\
Constante de tiempo de plato, min & 0.1 \\
& \\
\hline
\end{tabular}

(1) contando desde abajo
(2) sin incluir rehervidor y tanque de condensados 
A. Columnas de destilación

Tabla A.3. Datos en estado estacionario de la Columna C

\begin{tabular}{lc}
\hline Sistema & Benceno-Tolueno \\
\hline \hline Modelo Termodinámico & Ley de Raoult \\
Presión, atm & 1 \\
Composición de alimento, $C_{F}$ & 0.5 \\
Composición de destilado, $C_{D}$ & 0.98 \\
Composición de fondos, $C_{B}$ & 0.02 \\
Plato de alimentación $N_{F}(1)$ & 10 \\
N, número de platos ${ }^{(2)}$ & 0.6 \\
F, Kmol/min & 1.226 \\
V/F & 0.726 \\
R/F & 10 \\
Acumulado en condensados, Kmol & 10 \\
Acumulado en rehervidor, Kmol & 13.59 \\
Tiempo de residencia del condensador, min & 9.66 \\
Tiempo de residencia del rehervidor, min & 0.13 \\
Acumulado de plato, Kmol & Secc. Agotadora 0.367 \\
Constante de tiempo de plato, min & \\
\hline
\end{tabular}

(1) contando desde abajo

(2) sin incluir rehervidor y tanque de condensados 
A. Columnas de destilación

Tabla A.4. Datos en estado estacionario de la Columna D

\begin{tabular}{lc}
\hline Sistema & Metanol-Agua \\
\hline \hline Modelo Termodinámico & UNIFAC \\
Presión, atm & 1 \\
Composición de alimento, $C_{F}$ & 0.5 \\
Composición de destilado, $C_{D}$ & 0.99 \\
Composición de fondos, $C_{B}$ & 0.01 \\
Plato de alimentación $N_{F}(1)$ & 3 \\
N, número de platos ${ }^{(2)}$ & 12 \\
F, Kmol/min & 0.6 \\
V/F & 1.127 \\
R/F & 0.67 \\
Acumulado en condensados, Kmol & 10 \\
Acumulado en rehervidor, Kmol & 10 \\
Tiempo de residencia del condensador, min & 14.79 \\
Tiempo de residencia del rehervidor, min & 0.98 \\
Acumulado de plato, Kmol & 0.15 \\
Constante de tiempo de plato, min & Secc. Agotadora 0.367 \\
\hline \hline (1) contando desde abajo & \\
(2) sin incluir rehervidor y tanque de condensados & \\
& \\
&
\end{tabular}


A. Columnas de destilación

\section{A.2. $\boldsymbol{A} \boldsymbol{\operatorname { E n } n}(\tau)$ en función del tamaño de ventana $N_{w}$}

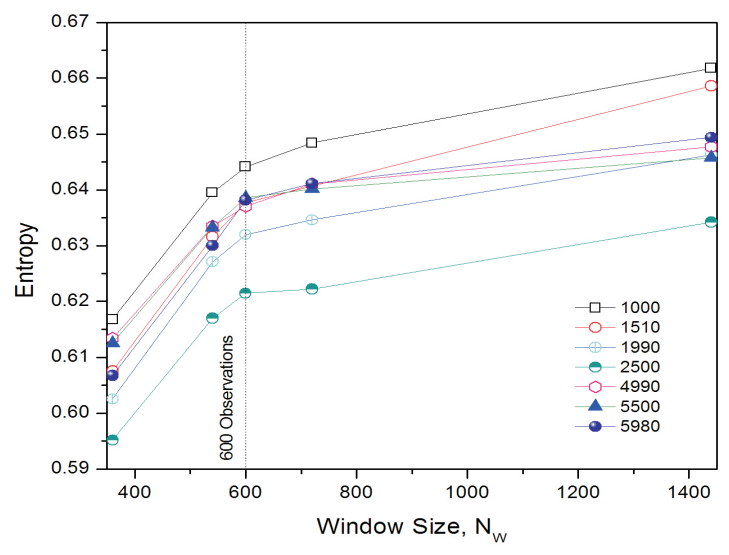

Figura A.1. Entropía aproximada $\operatorname{ApEn}(\tau)$ como una función de la longitud de la muestra $N_{s}$, para cuatro diferentes escalas de tiempo $\tau$ para la señal de temperatura de la columna C.

\section{A.3. Entropía aproximada multiescala}

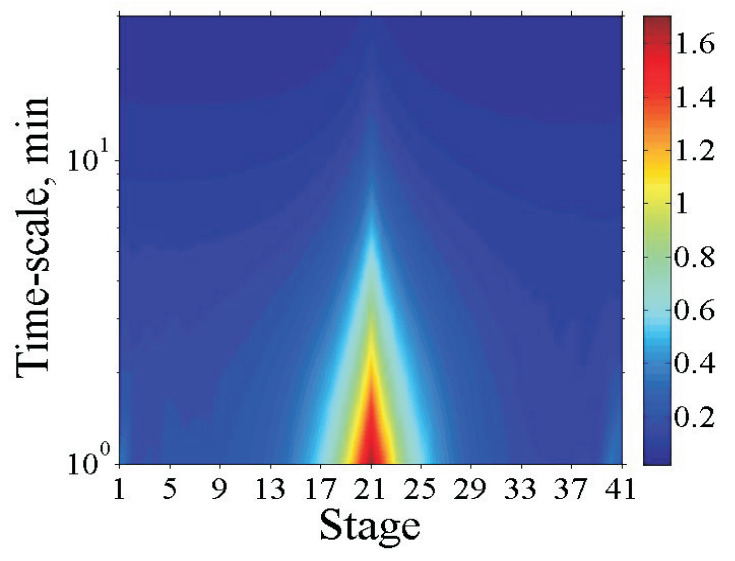

Figura A.2. Entropía aproximada multiescala de la columna de destilación A. 


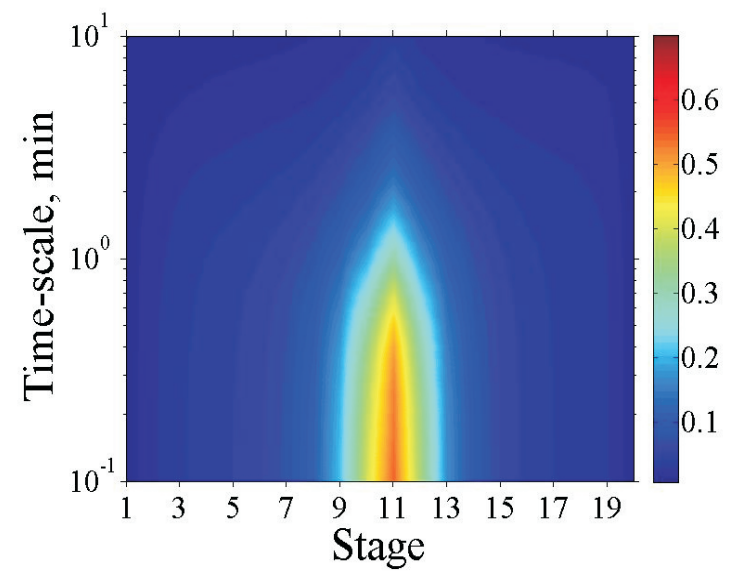

Figura A.3. Entropía aproximada multiescala de la columna de destilación B.

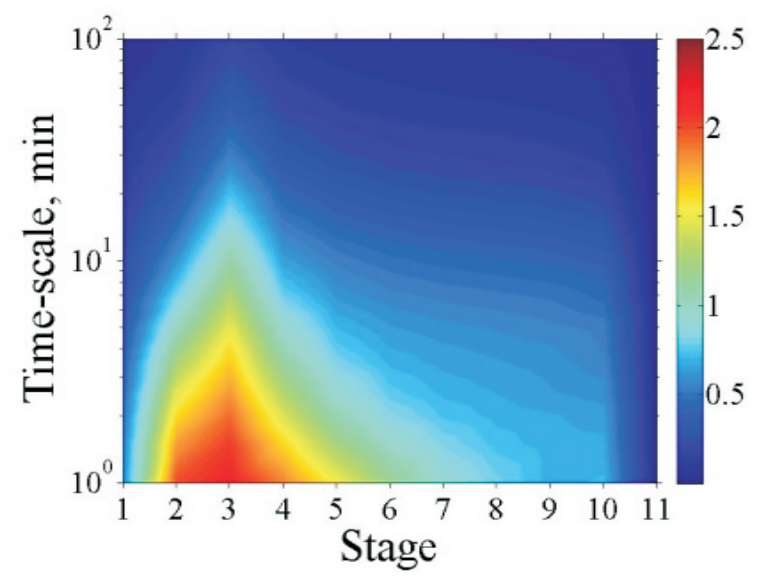

Figura A.4. Entropía aproximada multiescala de la columna de destilación D. 


\section{APÉNDICE B \\ ENTROPÍA APROXIMADA}

Pincus (1991) introdujo el estadístico de entropía aproximada (ApEn, por su acrónimo en inglés) para cuantificar la irregularidad y complejidad para datos experimentales de pocos datos y con ruido. La cuantificación de la entropía aproximada se basa en la probabilidad de que patrones en las series de tiempo que son similares permanecen similares en el siguiente incremento de comparación. Por lo tanto, las series de tiempo con altos valores de ApEn están asociadas a altas irregularidades (mayor complejidad) o mayores fluctuaciones, y de manera contraría series de tiempo con bajos valores de $A p E n$ se asocian a menores irregularidades (menor complejidad) o menores fluctuaciones.

El algoritmo de entropía aproximada $(A p E n)$ puede ser descrito como sigue:

Dada una serie de tiempo $\left\{X_{i}\right\}=\left\{x_{1}, x_{2} \ldots, x_{N}\right\}$ de longitud $N$, se define una secuencia de vectores $y^{(m)}(i)=\left\{x_{i}, x_{i+1} \ldots, x_{i+m-1}\right\}$. Dos vectores $y^{(m)}(i)$ y $y^{(m)}(j)$ son llamados similares si su distancia $d(i, j)=\max \{|x(i+k)-x(j+k)|: 0 \leq k \leq m-1\}$ es pequeña dada una tolerancia $r$. Para cada $N-m+1$ vectores $y^{(m)}(i)$ el número de vectores similares $y^{(m)}(j)$ es determinado por la medida de sus respectivas distancias. Sea $n_{i}^{(m)}$ el número de vectores similares a $y^{(m)}(i)$. La frecuencia relativa de encontrar un vector $y^{(m)}(j)$ que sea similar a $y^{(m)}(i)$ con un nivel de tolerancia $r$ es dada por: 


$$
C_{i}^{(m)}(r)=\frac{n_{i}^{(m)}}{N-m+1}
$$

Bajo la hipótesis del estacionario, se puede buscar que la frecuencia relativa sea el logaritmo de $C_{i}^{(m)}(r)$, por ejemplo,

$$
\Phi^{(m)}(r)=\frac{1}{N-m+1} \sum_{i=1}^{N-m+1} \ln C_{i}^{(m)}(r)
$$

La entropía se define como

$$
\operatorname{ApEn}(m, r)=\lim _{N \rightarrow \infty}\left(\Phi^{(m)}(r)-\Phi^{(m+1)}(r)\right)
$$

Para una muestra finita de longitud $N$, la $A p E n$ se estima con el estadístico

$$
\operatorname{ApEn}(m, r, N)=\Phi^{(m)}(r)-\Phi^{(m+1)}(r)
$$

Valores bajos de $\operatorname{ApEn}(m, r, N)$ reflejan series de tiempo más regulares mientras que valores altos se asocian con series de tiempo menos predecibles (más complejas). Pincus (1991) exploró los efectos de la tolerancia $r$, la longitud de los datos $N$ y la dimensión vectorial $m$ en el desempeño del cálculo computacional de la $A p E n$. Estadísticos estables se encontraron para $N>1000$. Por otra parte Pincus (1991) propuso el empleo de $m=2$ y $r=0.15 \sigma$, donde $\sigma$ es la desviación estándar de la serie de tiempo. Estos valores de los parámetros son ahora estándares en muchas aplicaciones de los algoritmos estándares para el cálculo de la entropía aproximada como el de las ecuaciones (B.1)-(B.4) y, a menos que se especifique lo contrario, serán empleados en los cálculos de entropía aproximada. 


\section{APÉNDICE C \\ ENTROPÍA APROXIMADA SIN TENDENCIA}

La hipótesis del estacionario es una definición que debe aplicarse en los cálculos computacionales de ApEn. Sin embargo, los datos reales complejos no exhiben un estado estacionario; y con frecuencia pueden llevar a interpretaciones erróneas en la estimación de la $A p E n$. En muchos casos, las no estacionalidades son inducidas por fuerzas exógenas que actúan en la dinámica del sistema, provocando largas tendencias en el comportamiento de las series de tiempo.

En este trabajo de investigación se ha introducido un análisis de entropía aproximada sin tendencia $D E A$ para remover los efectos de tendencia en series de tiempo reales complejas. Para conseguir lo anterior, la serie de tiempo original, $\left\{X_{i}\right\}=\left\{x_{1}, x_{2} \ldots, x_{N}\right\}$ de longitud $N$, se dividió en $M=[N / L]$ cajas que no se sobreponen de muestras de longitud $L$. Si $\Delta t$ es el tiempo de muestreo, $\tau=L \Delta t$ representa la escala de tiempo. En cada ventana de tiempo, una línea de mínimos cuadrados ajusta los datos, la cual representa la tendencia en esa ventana. La coordenada vertical del segmento de línea se $x_{i}^{(\tau)}$. Al remover la tendencia en la serie de tiempo original se obtiene una serie de tiempo sin tendencia $\left\{Z^{(\tau)}(i)\right\}$ correspondiente a una escala de tiempo $\tau$ :

$$
z_{i}^{(\tau)}=x_{i}-x_{i}^{(\tau)}, \quad i=1,2, \ldots
$$


C. Entropía aproximada sin tendencia

Los siguientes pasos corresponden a la aplicación del algoritmo para calcular la $A p E n$, Apéndice B, mediante las ecuaciones (B.1)-(B.4) para las series de tiempo sin tendencia $\left\{Z^{(\tau)}(i)\right\}$. El procedimiento se repite para todas las escalas de tiempo (tamaños de caja) para caracterizar las variaciones de la $A p E n$ con la escala de tiempo. Es decir, en el proceso se elimina la tendencia unida con el procedimiento de calcular computacionalmente la $A p E n$ estándar. Lo que conduce naturalmente a la caracterización multiescala de la complejidad de la serie de tiempo cuantificada en términos de la funcionalidad de la entropía multiescala $\operatorname{Ap} \operatorname{En}(\tau)$.

Al aplicar el procedimiento del $D E A$ descrito anteriormente e interpretar los resultados se deben de considerar los siguientes comentarios:

El paso de eliminación de la tendencia actúa como un filtro pasa altas al remover componentes de frecuencia menores que $\tau^{-1}$. De esta manera, $D E A(\tau)$ debe reflejar la regularidad de la series de tiempo para frecuencias no menores que $\tau^{-1}$.

Debe observarse que la tolerancia está dada en términos de la desviación estándar de la serie de tiempo sin tendencia en el sentido que $r(\tau)=\rho \sigma(\tau)$, para una valor fijo de $\rho \epsilon(0,1)$. Sin embargo, $\sigma(\tau)$ no depende de la varianza de la serie de tiempo original, por ejemplo, el valor absoluto de los elementos de los datos. En cambio si se usa una tolerancia fija, se estaría midiendo también la desviación estándar y no sólo la entropía.

Se sugiere limitar los cálculos de la $D E A$ para cajas de tamaño $L \geq 10$. Cálculos computacionales de la $A p E n$ con una preferencia se pueden obtener para cajas de tamaño menores debido a efectos del muestreo de tamaño finito. 


\section{APÉNDICE D ENTROPÍA APROXIMADA MULTIESCALA}

En general, la complejidad de sistemas reales no está restringida a una sola escala, esto es, pueden exhibir patrones complejos temporales en un amplio rango de escalas de tiempo. De esta forma, la entropía es una medida del promedio de incertidumbre en una serie de tiempo y es dependiente de la escala.

Lo que significa que una señal puede aparecer con más incertidumbre en una escala de tiempo y más regular en otras. Por lo cual, una caracterización completa de entropía para series de tiempo debe considerar la variabilidad a través de rangos de escalas no triviales. La idea fundamental de un enfoque de entropía multiescala deberá consistir en proporcionar un índice que refleje la velocidad media de creación de información a una escala dada. Como resultado, el grado total de predictibilidad e incertidumbre de una señal es evaluado considerando los valores de entropía estimados para un rango predefinido de escalas de tiempo. El método de entropía aproximada multiescala involucra dos pasos:

1. Un método que permite visualizar la representación de un sistema dinámico a diferentes escalas de tiempo. Dada una serie de tiempo $X=\left\{x_{1}, x_{2}, \ldots, x_{N}\right\}$, y un procedimiento de filtro pasa-bajas $L P(f)$ con una frecuencia de corte $f$, se obtienen series de tiempo filtradas como $Y_{f}=L P(f) \cdot X$, donde $Y_{f}=\left\{y_{f, 1}, y_{f, 2}, \ldots, y_{f, N}\right\}$. De esta forma, las nuevas series de tiempo $Y_{f}$ conservan la complejidad de la señal $X$ para frecuencias 
menores que $f$, o escalas de tiempo mayores que $\tau=\frac{1}{f}$. Diferentes esquemas de filtros pasa-bajas $L P(f)$ están disponibles en paquetes comerciales. En este trabajo de investigación, al igual que en los análisis técnicos de mercados para obtener tendencias a largo plazo de señales financieras, el filtro pasa-bajas se realizó con filtros de promedio móvil. De esta forma, la señal filtrada es obtenida como sigue:

$$
Y_{f, i}=\frac{1}{n} \sum_{j=1}^{n} x_{i+j-1}
$$

La escala de tiempo está dada por $\tau=\Delta t n$ y $f=\frac{1}{\tau}$. Donde, $\Delta t$ es el periodo de muestreo.

2. La cuantificación del grado de irregularidad de cada una de las series de tiempo $Y_{f}$ es realizado usando el procedimiento de entropía aproximada descrito en el Apéndice B. 